Review

\title{
Mechanisms underlying the generation of autonomorespiratory coupling amongst the respiratory central pattern generator, sympathetic oscillators, and cardiovagal premotoneurons
}

The respiratory rhythm and pattern and sympathetic and parasympathetic outflows are generated by distinct, though overlapping, propriobulbar arrays of neuronal microcircuit oscillators constituting networks utilizing mutual excitatory and inhibitory neuronal interactions, residing principally within the metencephalon and myelencephalon, and modulated by synaptic influences from the cerebrum, thalamus, hypothalamus, cerebellum, and mesencephalon and ascending influences deriving from peripheral stimuli relayed by cranial nerve afferent axons. Though the respiratory and cardiovascular regulatory effector mechanisms utilize distinct generators, there exists significant overlap and interconnectivity amongst and between these oscillators and pathways, evidenced reciprocally by breathing modulation of sympathetic oscillations and sympathetic modulation of neural breathing. These coupling mechanisms are well-demonstrated coordinately in sympatheticand respiratory-related central neuronal and efferent neurogram recordings and quantified by the findings of crosscorrelation, spectra, and coherence analyses, combined with empirical interventions including lesioning and pharmacological agonist and antagonist microinjection studies, baroloading, barounloading, and hypoxic and/or hypercapnic peripheral and/or central chemoreceptor stimulation. Sympathetic and parasympathetic central neuronal and efferent neural discharge recordings evidence classic fast rhythms produced by propriobulbar neuronal networks located within the medullary division of the lateral tegmental field, coherent with cardiac sympathetic nerve discharge. These neural efferent nerve discharges coordinately evidence slow synchronous oscillations, constituted by Traube Hering (i.e., high frequency), Mayer wave (i.e., medium or low frequency), and vasogenic autorhythmicity (i.e., very low frequency) wave spectral bands. These oscillations contribute to coupling neural breathing, sympathetic oscillations, and parasympathetic cardiovagal premotoneuronal activity. The mechanisms underlying the origins of and coupling amongst, these waves remains to be unresolved.

\section{Keywords}

Mechanisms; genesis; sympathorespiratory; coupling; modulation; cardiovagal; rostral ventrolateral medulla; sympathetic; hypercapnia; hypoxia 


\section{Mechanisms underlying generation of the breathing rhythm and pattern, sympathetic oscillations, and cardiovagal premotoneuronal activity}

We contextualize a discussion of autonomorespiratory (e.g., sympathorespiratory, parasympathorespiratory, sympathoparasympathetic) coupling (Guyenet et al., 2019; Molkov et al., 2014; Zoccal, 2015) by evaluating the mechanisms generating the individual activities proper (Ghali, 2017a,b,c, 2019a,b,c, 2018) (Figs. 1-14). Distinct, though overlapping, propriobulbar neuronal microcircuit oscillators disparately distributed throughout and specifically organized within, the brainstem and upper cervical spinal cord emergently constituting brainstem and myelic subnetworks and networks (Goodchild and Moon, 2009; Guyenet et al., 2018; Molkov et al., 2017) generate neural breathing (Figs. 1-3) (Anderson et al., 2016; Anderson and Ramirez, 2017; Ghali and Marchenko, 2013, 2015, 2016a,b; Marchenko et al., 2016; Richter, 1982; Richter and Spyer, 1990; Richter et al., 1986) and basal and reflexive changes in sympathetic and cardiovagal tone (Figs. 4 and 5). Multiple distinct (Anderson and Ramirez, 2017) discretely (Molkov et al., 2017) organized metencephalic and myelencephalic nodes constituted by propriobulbar and bulbospinal units exhibiting respiratory-related modulation (Marchenko et al., 2016) RTN (Guyenet et al., 2019); postinspiratory complex (Anderson et al., 2016) coupled by excitatory and inhibitory interneuronal interactions emergently generate triphasic eupnea (defined by Richter (1982); see Richter et al. (1986)) characterizing neural breathing (Ramirez and Baertsch, 2018) and sympathetic and parasympathetic oscillations modulating arterial and venous tone (Dittmar, 1873; Ghali, 2017a, 2018; Gordon and McCann, 1988; Guyenet, 2006) and myocardial contraction force and frequency (Lindsey et al., 1998; Massari et al., 1998), effectively modulating dynamic arterial and venous pressure, resistance, and flow and sinoatrial rate, atrioventricular conduction, atrial and ventricular contractility, output, and function (Ghali, 2017a, 2018; Guyenet, 2006).

Two bilateral rostrocaudally organized columns of nuclei residing within the ventrolateral and dorsal medulla (Figs. 1-3) (Molkov et al., 2017; Ramirez and Baertsch, 2018; Smith et al., 2009), receiving excitatory and inhibitory tonic and phasic modulatory inputs from chemosensitive neurons residing in the retrotrapezoid nucleus (Figs. 1 and 5) (Guyenet et al., 2019) and propriobulbar modulatory synaptic drive from Kölliker-Fuse and medial parabrachial nuclei in the dorsolateral metencephalic tegmentum (Dutschmann and Herbert, 2006; Mörschel and Dutschmann, 2009) emergently generate breathing. Interactions of neurons within the preBötzinger (preBötzC) (Gourévitch and Mellen, 2014; Gray et al., 2010; MorgadoValle et al., 2010) and Bötzinger (BötzC) (Bongianni et al., 2010; Marchenko et al., 2016) complexes generate a two-phase (i.e., inspiratory and expiratory) neural respiratory rhythm. BötzC decrementing post-inspiratory neurons (Ezure and Manabe, 1988) receiving propriobulbar modulatory excitatory synaptic drive from KöllikerFuse and medial parabrachial nuclei in the dorsolateral metencephalic tegmentum (Dutschmann and Herbert, 2006; Mörschel and Dutschmann, 2009), reciprocally interacting with BötzC augmenting late-expiratory neurons via inhibitory neurosynaptic interactions (Smith et al., 2009), segregate expiration into post-inspiratory and late-expiratory phases generating triphasic eupnea. The core rhythm distributes to premotoneurons residing within the rostral and caudal divisions of the ventral respiratory group (see Tian and Duf- fin (1996a,b)). The dorsal respiratory group (see Richardson and Mitchell (1982)), monosynaptically or polysynaptically projecting to and synaptically driving inspiratory-related motoneurons residing within the brainstem (Ghali, 2019a; van Brederode and Berger, 2011) and spinal cord (Christakos et al., 1988, 1991; Marchenko et al., 2012). Ventral respiratory group brainstem interneurons project to, and putatively modulate the discharge of, neurons residing within the ventrolateral division of the nucleus tractus solitarius (NTS), both zones of which are coupled across the midline by propriobulbar units (Iakunin et al., 1982) and are critically implicated in the genesis of high-frequency oscillations (Romaniuk and Bruce, 1991), evident in respiratory-related neuronal and neural efferent spectra (Christakos et al., 1991; Ghali and Marchenko, 2013; Marchenko et al., 2012). We suggest these synaptic connections modulate mutual sympathorespiratory coupling across sympathetic and breathing oscillators.

The central pattern generator originating the breathing rhythm (see Von Euler et al. (1973)) receives powerful modulatory synaptic drive by central and peripheral receptors sensitive to oxygen (Garcia et al., 2016) and/or carbon dioxide tension (Ghali and Marchenko, 2016b; Guyenet et al., 2019), alveolar stretch (Breuer, 1868; Dutschmann et al., 2014; Hering, 1868; Luck, 1969), laryngeal fibers (D'iachenko and Preobrazhenskiǔ, 1984a,b), and arterial stretch (Baekey et al., 2010; Bishop, 1974; Lindsey et al., 1998; Morrison et al., 1984). Afferent inputs to propriobulbar interneuronal microcircuit oscillators generating the sympathetic and respiratory neural activities accordingly derive from higher-order neurons of the nucleus tractus solitarius (Fig. 5) (Jean, 1992). These influences monomodally and multimodally integrate and filter oscillatory sensory inputs centrally conveyed by peripheral afferents innervating the carotid sinus and body and aortic arch baroreceptors and chemoreceptors and diffusely distributed central chemoreceptors (Michelini, 1994; Moreira et al., 2007; Rogers et al., 2000; Takakura et al., 2007) located within the brainstem and cerebellum (Mulkey et al., 2006). Drugs modulating sympathetic neural discharge and arterial pressure may coordinately modify the activity of the central sympathetic oscillators, autonomic ganglia, and peripheral effectors (Gillis, 1995). Propriobulbar interneuronal microcircuit oscillators constituting the respective central pattern generators critically gate incoming central and peripheral sensory influences conveyed by afferent neuronal synaptic inputs (Baev et al., 1981), a principle well demonstrated in brainstem vestibular networks (Marlinskiı̌ et al., 1989; Preobrazhenskiı̌ et al., 1989; Shumilina and Preobrazhenskiı̌, 1991) and spinal cord substantia gelatinosa. Evidence suggests propriospinal interneuronal microcircuit oscillators may be capable of generating rhythmic activity in respiratory-related neural and motor outputs (Ghali and Marchenko, 2016a).

The majority of neurophysiologists presume sympathetic activity to originate within the propriobulbar and presympathetic neuronal circuitry of the rostral ventrolateral medulla (Fig. 3) (Guyenet et al., 2018). These zones are constituted chiefly of glutamatergic (VGlut2 positive) and catecholaminergic (tyrosine hydroxylase immunoreactive) cells. Though the RVLM propriobulbar and presympathetic neurons putatively generate sympathetic activity, evidence more strongly favors principal genesis by medullary lateral tegmental field propriobulbar interneurons (Ghali, 2018), a wise evolutionary construct in the event of ischemic injury (personal communication, V. Marchenko). Accordingly, early experiments interrogating brainstem circuitry generating sympathetic activity pro- 


\section{Medullary Drive to Phrenic Nucleus}

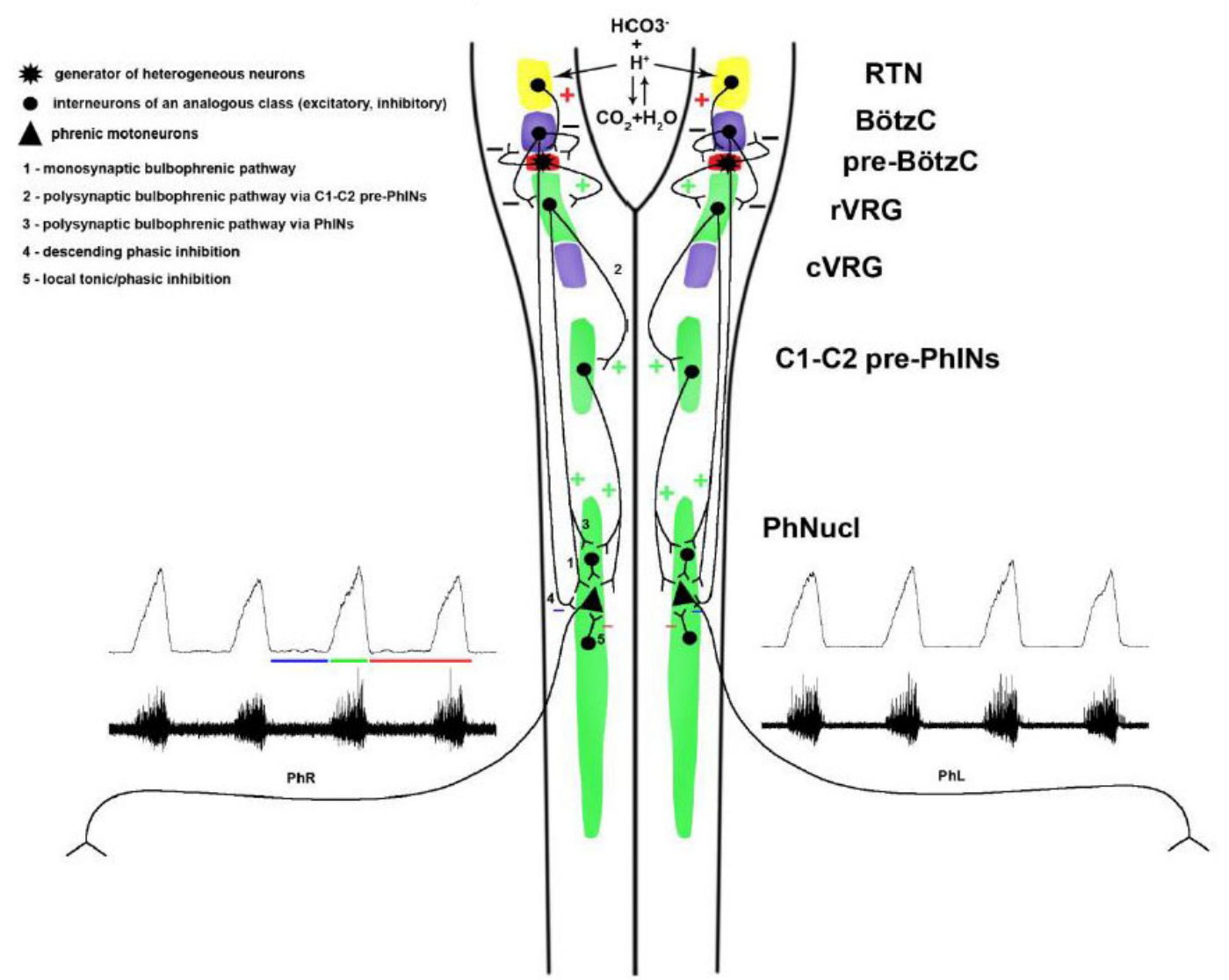

Fig. 1. Conceptual model of brainstem neuronal micro-oscillator synaptic drive to the phrenic motor nucleus. A discrete embryonically homogenous cluster of Phox $2 b$ transcription factor-expressing chemosensitive neurons extant near the pontomedullary confluence constitutes the retrotrapezoid nucleus. Hydrogen ion concentration dynamically parallels dynamic retrotrapezoid nucleus glutamatergic neuronal spiking frequency. Hydrogen ions generated by the reaction of arterial $\mathrm{CO}_{2}$ with $\mathrm{H}_{2} \mathrm{O}$ generate the weak acid $\mathrm{H}_{2} \mathrm{CO}_{3}$, which exists in equilibrium with its constituent dissociation products $\mathrm{H}^{+}$cation and $\mathrm{HCO}_{3}{ }^{-}$cation. The $\mathrm{HCO}_{3}{ }^{-}$anion dissociates into $\mathrm{H}^{+}$and $\mathrm{CO}_{3}{ }^{2-}$, with an equilibrium preferentially favoring the formation of $\mathrm{HCO}_{3}{ }^{-}$. These equilibria and dynamics are governed by the Henderson-Hasselbach proportionality, $\mathrm{pH}=\mathrm{pK}_{a}+\log \left(\left[\mathrm{H}^{+}\right]\left[\mathrm{A}^{-}\right] /[\mathrm{HA}]\right)$. Retrotrapezoid nucleus glutamatergic units convey prominent tonic excitatory drive and support to propriobulbar and bulbospinal microcircuit oscillators constituting the Bötzinger and pre-Bötzinger complexes, ventral respiratory column nuclei, dorsal respiratory group, and metencephalic elements constituting the brainstem neural respiratory network. Neurons exhibiting differential phase preference extant within the Bötzinger and pre-Bötzinger complexes dynamically interact to generate a core two-phase neural respiratory rhythm and modulate premotoneuronal spiking frequency in the rostral and caudal divisions of the ventral respiratory group. BötzC dec post-I and aug late-E units convey inhibitory modulation to preBötzC pre-I, pre-I/I, and dec early-I units and propriobulbar excitatory drive to cVRG expiratory premotoneurons. PreBötzC pre-I, pre-I/I, and dec early-I units reciprocally inhibit BötzC dec post-I and aug-E cells. PreBötzC excitatory pre-I and pre-I/I units monosynaptically and paucisynaptically drive rVRG aug-I units. PreBötzC inhibitory dec early-I units putatively shape inspiratory ramp by inhibiting the rVRG aug-I premotoneuronal driver population during the early phase of the inspiratory epoch. Rostral ventral respiratory group premotoneurons drive the phasic activity of phrenic motoneurons through projections conveyed through ipsilateral (i.e., pathways which do not decussate or decussate twice across the midline at medullary then upper $\mathrm{C}_{1}-\mathrm{C}_{2}$ cervical spinal cord or phrenic motoneuronal levels) and contralateral (pathways which decussate once at either brainstem, upper cervical spinal cord, or phrenic motor nucleus levels) ventromedial and lateral funiculi of the myelic substance relaying to phrenic motoneurons monosynaptically or through interposed pre-phrenic interneurons located in the upper cervical spinal cord or phrenic motor nucleus. Phrenic motoneuronal neurites crossing the midline into the contralateral hemicord may receive descending axodendritic and axosomatic inputs from rostral ventral respiratory group axonal terminals conveyed through the ventromedial and lateral funiculi of the spinal cord. Phrenic motoneurons with dendrites decussating across the midline constitute a significant fraction of these units during early neonatal age, and evidence rapid age-dependent decreases. Medullophrenic units (chiefly from BötzC or Kölliker-Fuse nucleus bulbospinal cells) may convey phasic inhibitory modulation of PhMNs. Local pre-phrenic interneurons may coordinate convey phasic inhibition and convey tonic inhibitory modulation of PhMNs. Color traces beneath phrenic neurograms indicate the phase of activity (i.e., inspiratory, expiratory [post-I and E2], or tonically discharging units) of indicated excitatory (+) and inhibitory (-) synapses. RTN, retrotrapezoid nucleus; BötzC, Bötzinger complex; preBötzC, preBötzinger complex; rVRG, rostral ventral respiratory group; cVRG, caudal ventral respiratory group; $\mathrm{C}_{1}-\mathrm{C}_{2}$ pre-PhINs, $\mathrm{C}_{1}-\mathrm{C}_{2}$ pre-phrenic interneurons; PhNucl, phrenic nucleus; $\mathrm{PhL}$, left phrenic nerve; PhR, right phrenic nerve. Modified with permission from Fig. 10 of Ghali and Marchenko (2016a) 
vided initial evidence indicating a diffuse distribution of medullary units exhibiting sympathetic-correlated discharge (Preobrazhenski1, 1966). A robust set of data validates genesis of sympathetic activity derives from synchronized activity among sympathoexcitatory and sympathoinhibitory propriobulbar interneuronal microcircuit oscillators residing within the medullary lateral tegmental field (Fig. 4) (Barman, 2020; Dampney, 1994; Gebber and Barman, 1988; Ghali, 2018; Marchenko and Sapru, 2003), thence conveyed to diffusely distributed presympathetic neurons residing within the rostral ventrolateral and ventromedial medulla (Babic and Ciriello, 2004), caudal raphé, and ventrolateral metencephalic tegmentum projecting to and driving neuronal spiking of, intermediolateral cell column preganglionic sympathetic neurons (Barman, 2020; Ghali, 2017a, 2018; Gilbey et al., 1995; Zhou and Gilbey, 1992). Medullary lateral tegmental field neurons oscillate with frequencies of 2 to $6 \mathrm{~Hz}$ and $10 \mathrm{~Hz}$, correlated with analogous spectra in cardiac sympathetic nerve discharge precede and drive similar activities in rostral ventrolateral medullary units (Barman, 2020; Ghali, 2018). Rostral ventromedial medullary presympathetic bulbospinal neurons specifically convey synaptic drive to intermediolateral cell column preganglionic sympathetic neurons projecting to postganglionic sympathetic neurons modulating cutaneous vasoconstrictor tone and thus contributing prominently to thermoregulation (see Guyenet (2006)) for outstanding review). Rostral ventrolateral medullary presympathetic neurons receive glutamatergic axodendritic, and axosomatic synaptic inputs drive biophysically transduced by N-methyl-D-aspartate (NMDA), $\alpha$-amino-3-hydroxy-5methyl-4-isoxazolepropioinic acid (AMPA), and kainate-type glutamate receptors. $\alpha_{2}$ autoreceptors localizing to the presynaptic axonal membranes of propriobulbar units exhibiting oscillations correlating with cardiac sympathetic nerve discharge negatively modulate the release of excitatory neurotransmitters from axonal terminals, mitigating the release of excitatory amino acid-mediated electrochemical amplification of somatodendritic conductance and contributing to hypotensive effects generated by administration of clonidine and $\alpha$-methyldopa. Sympathetic-related propriobulbar units receive prominent tonic inhibitory GABAergic and glycinergic modulation from specific nuclearly-arranged neuronal clusters and disparately distributed units located in surrounding reticular zones (Gatti et al., 1987). Neurogenesis of sympathetic nerve activity and rhythmic discharge may alternately be generated by a network (Lipski et al., 1996a,b) and/or pacemaker (Sun and Guyenet, 1986; Sun et al., 1988) mechanisms, organized by the medullary lateral tegmental field (Barman et al., 2005; Barman, 2020; Dampney, 1994; Ghali, 2017a; Marchenko and Sapru, 2003), rostral ventrolateral medulla (Guyenet, 2006), and distributed regions within the brainstem (Figs. 5) (Ghali, 2018; Goodchild and Moon, 2009), neuroanatomically overlapping and interacting with the neuronal propriobulbar circuitry generating and organizing neural breathing (Figs. 4 and 5) (see Anderson and Ramirez, 2017; Molkov et al., 2017, 2014).

Diffusely distributed nuclearly arranged neuronal clusters residing within functionally distinct subnuclei of the nucleus tractus solitarius (Fig. 5) (Michelini, 1994; Moreira et al., 2007; Takakura et al., 2007; Zhao et al., 1997) play modulatory roles in, and constitute the chief nodes mediating and contributing to respiratory, sympathetic, and parasympathetic crossmodal modulation and coupling (Guyenet, 2006; Molkov et al., 2014). Nucleus tractus solitarius units monomodally filter and/or multimodally integrate a wide array of propriobulbar interneuronal inputs and axon terminals from peripheral afferent fibers, coordinately conveying monosynaptic and polysynaptic inputs to higher-order neurons (Fig. 5) (Von Euler et al., 1973), coordinately negatively modulating propriobulbar interneuronal sympathetic- and inspiratory-related microcircuit oscillators and positively modulating propriobulbar interneuronal cardiovagal- and expiratory-related microcircuit oscillators (Guyenet, 2006; Moreira et al., 2007; Rogers et al., 2000; Takakura et al., 2007). Dorsal respiratory group bulbospinal premotoneurons convey axodendritic and axosomatic synaptic drive to the brainstem and spinal cord respiratory-related motoneurons (Lipski et al., 1990). Nucleus ambiguus (i.e., N.A.) and dorsal motor nucleus of the vagus (DMV) convey cardiovagal preganglionic neurons en route to cardiac plexus ganglia postganglionic parasympathetic neurons powerfully modulating sinoatrial chronotropy, atrioventricular dromotropy, and atrial inotropy (Dyavanapalli et al., 2013; Eckberg, 2003; Taylor et al., 2009), though a few fibers coordinately supply ventricular myocardium (Coote, 2013). Postganglionic sympathetic and parasympathetic cardiovagal axons modulate sinoatrial action potential frequency by influencing the slope of spontaneous depolarization mediated by persistent sodium channel current in sinoatrial nodal cells, atrioventricular conductivity by regulating the slope of voltage-gated calcium current rise in atrioventricular nodal cells, and atrial and ventricular contractility by determining magnitude and rate of rising of cytosolic calcium flux deriving from the extracellular space and sarcoplasmic reticulum stores (Ghali, 2017a, 2018). The data collectively substantiates monomodal and multimodal sensory influences effectively converge upon, diverge through, and modulate the discharge of, brainstem sympathetic oscillators and cardiovagal premotoneurons (Ghali, 2017a, 2018). Visceromotor (Duda et al., 1970a,b; Kostiuk and Preobrazhenskiǔ, 1966; Preobrazhenskiǔ et al., 1972; Preobrazhenskiı̌ and Tamarova, 1966), somatosympathetic (Ghali, 2019a), and somatorespiratory (Ghali, 2019a) reflexes exhibit sympathosomatic and respirosomatic coupling and extensive interconnectivity amongst propriobulbar interneuronal networks generating and mediating nociceptive responses, sympathetic discharge, and the breathing rhythm.

Prefacing a discussion of autonomorespirophasic modulation (Figs. 5-14), we briefly discuss the neural network and pacemaker mechanisms emergently generating the breathing rhythm and pattern (Molkov et al., 2017), sympathetic oscillations (Barman, 2020; Ghali, 2017a, 2018; Molkov et al., 2014), and cardiovagal premotoneuronal discharge (Bishop, 1974). We discuss respirophasic modulation of brainstem presympathetic neuronal oscillations (Guyenet et al., 1990; Haselton and Guyenet, 1989; Lipski et al., 1996a,b; McAllen, 1987; Miyawaki et al., 1996; Numao et al., 1987), sympathetic preganglionic and postganglionic discharge (Guyenet et al., 1990; Mandel and Schreihofer, 2006; Zhou and Gilbey, 1992) and cardiovagal premotoneuronal spiking (Gilbey et al., 1984; Lindsey et al., 1998). We reciprocally discuss oscillatory baroreceptor modulation of the breathing pattern (Baekey et al., 2010; Bishop, 1974; Brunner et al., 1982; Dove and Katona, 1985; Lindsey et al., 1998) and respiratory gating of the baroreflex (Baekey et al., 2010).

\section{Sympathorespiratory coupling}

Sympathorespiratory coupling was initially described by Adrian and Bronk in 1932 in the anesthetized rabbit (Adrian et al., 1932), 


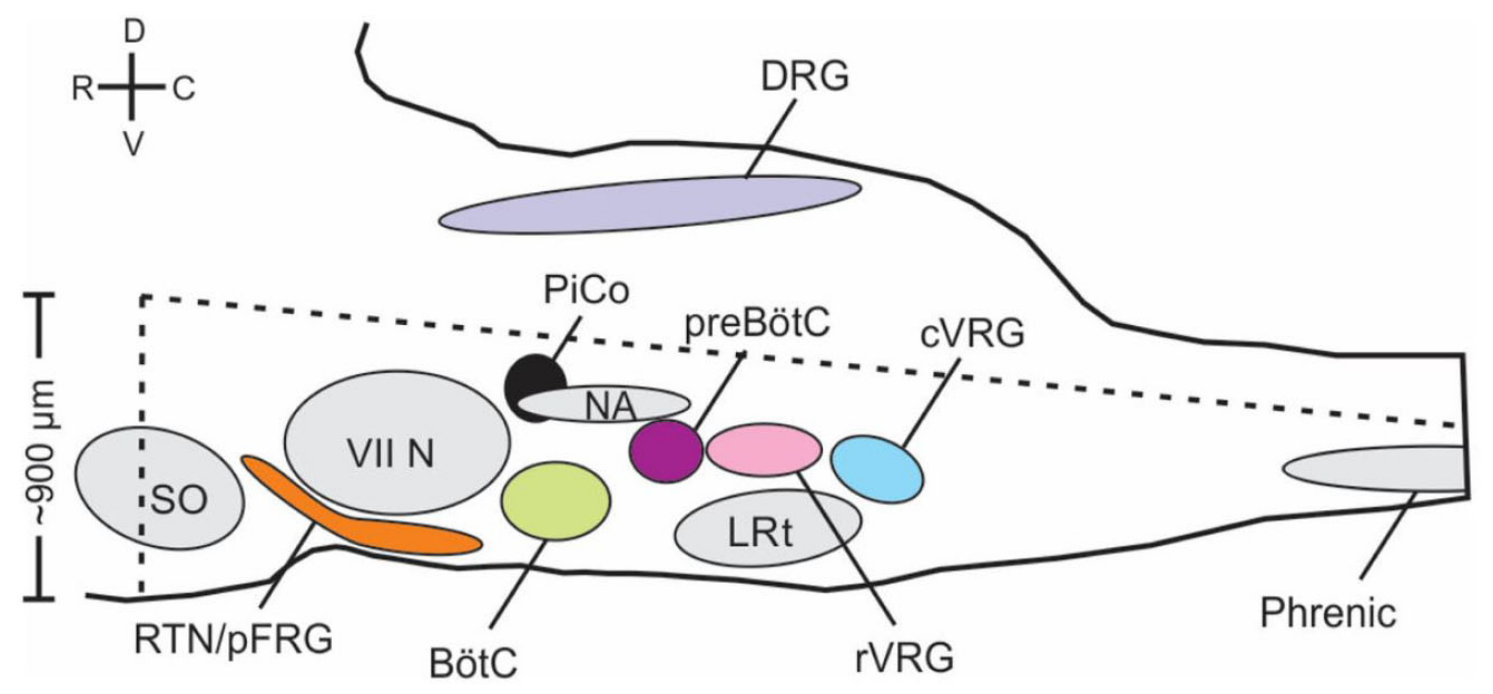

Fig. 2. Brainstem oscillators generating breathing rhythms. Cells distributed along the ventrolateral column of pontomedullary nuclei generate the neural breathing rhythm and pattern. The retrotrapezoid nucleus/parafacial respiratory group (RTN/pFRG), lies ventral with respect to the facial nucleus (VII). The postinspiratory complex ( $\mathrm{PiCo}$ ) may be found caudal to the facial nucleus, rostral to the preBötzinger complex, and dorsomedial with respect to the rostral pole of the nucleus ambiguus (NA). The Bötzinger (BötC) and preBötzinger complexes (preBötC) may be found ventromedial to the nucleus ambiguus and rostral with respect to the rostral (rVRG) and caudal (cVRG) divisions of the ventral respiratory group. The lateral reticular nucleus (LRt) conceals the preBötzC, rVRG, and cVRG from the ventral surface of the medulla. We presume lateral reticular nucleus neuronal somata constitute interposed relay phrenicomedullary inputs from the phrenic nucleus to the bulbar ventral respiratory column. Analogously, the serpiginous dorsal margin of the inferior olive separates the ventral respiratory group from the ventral surface of the brainstem in humans. Compartmental borders containing the ventral respiratory column nuclei are not markedly distinct. Electrophysiological properties and differential spatiotemporal firing dynamics have characteristically distinguished these regions, though investigators continue to strive to identify specific cell surface proteins and transcription factors distinguishing these zones. PreBötzinger complex neurons frequently express SST1 and/or the transcription factor Dbx1. Modified with permission from (Fig. 1) of Anderson et al. (2016)

demonstrating prominent differential modulation of the sympathetic neural efferent discharge varying according to the respiratory cycle, though perhaps previously intimated by studies conducted in the 1800s. Notably, cervical sympathetic burst amplitude increased markedly and dynamically toward the end of the inspiratory epoch (Adrian et al., 1932). Studies have continued to provide evidence indicating respirophasic modulation of sympathetic neural efferent activity, with variable accentuations and troughs of sympathetic bursting corresponding with precise intervals within the inspiratory and expiratory epochs (Molkov et al., 2014; Moraes et al., 2012a,b,c; Zoccal, 2015; Zoccal and Machado, 2011). Reciprocally, respiratory-related neuronal and neural outputs exhibit baromodulation and strong correlation with dynamic arterial pressure magnitude (Darnall and Guyenet, 1990; Gilbey et al., 1986; Lipski et al., 1996a,b; McAllen, 1987; Zhou and Gilbey, 1992). Sympathorespiratory coupling occurs during eucapnic normoxia (Adrian et al., 1932), though it becomes strengthened by hypoxia and hypercapnia (Moraes et al., 2012a,b,c; Zoccal, 2015; Zoccal and Machado, 2011).

Crossmodal modulation of neural breathing, sympathetic oscillations, and cardiovagal premotoneuronal spiking couples the activities (Gilbey et al., 1995; McAllen, 1987; Molkov et al., 2014; Zoccal, 2015; Zoccal and Machado, 2011). Propriospinal microcircuit oscillators constituting emergently dynamic networks generate the respiratory rhythm and pattern (Figs. 1-3) (Ghali and Ghali, 2020), sympathetic oscillations (Figs. 4 and 5) (Barman, 2020), and cardiovagal premotoneuronal activity (Figs. 4 and 5) (Frank et al., 2009). The breathing cycle modulates rostral ventrolateral medullary sympathetic propriobulbar and presympathetic units
(Gilbey et al., 1995; Lipski et al., 1996a,b; Mandel and Schreihofer, 2006; McAllen, 1987; Miyawaki et al., 1996; Terui et al., 1986), and spinal cord preganglionic sympathetic neurons (Zhou and Gilbey, 1992) (Fig. 7) sympathetic neural efferent nerve discharge (Fig. 6). Reciprocally, sympathetic and baroreceptor oscillations modulate respiratory-related brainstem and spinal cord neuronal spiking pattern (Baekey et al., 2010; Bishop, 1974; Brunner et al., 1982; Dove and Katona, 1985; Eckberg and Orshan, 1977; Grunstein et al., 1975; Lindsey et al., 1998). A large fraction of respiratory-related units recorded in unanesthetized decerebrate vagotomized cats exhibits pulse-synchronous modulation (Lindsey et al., 1998). Accentuations or attenuations of neuronal or neural efferent spiking frequency or burst clustering occurring in response to synaptic inputs conveyed by another group of neurons constitutes modulation (Gilbey et al., 1995; Lindsey et al., 1998; Zhou and Gilbey, 1992). We designate a rostroventrolateral medullary presympathetic neuronal unit exhibiting peak spiking frequency during the inspiratory epoch of breathing to possess inspiratory modulation (Lipski et al., 1996a,b; Mandel and Schreihofer, 2006; Miyawaki et al., 1996).

During normoxic normocapnia, autonomorespiratory crossmodal modulation chiefly originates from the provision of coordinate inputs deriving from higher-order neural nets (Abdala et al., 2009; Moraes et al., 2012b) and barosensitive, chemosensitive, and pulmonary stretch mechanosensitive monomodal and multimodal nucleus tractus solitarius units (Moreira et al., 2007; Takakura et al., 2007) and direct interaction amongst intermingled propriobulbar interneuronal microcircuits constituting the respiratory central pattern generator, sympathetic oscillators, and dorsal medullary cardiovagal premotoneurons (Moraes et al., 2014a,b, 2012c; Sun et al., 


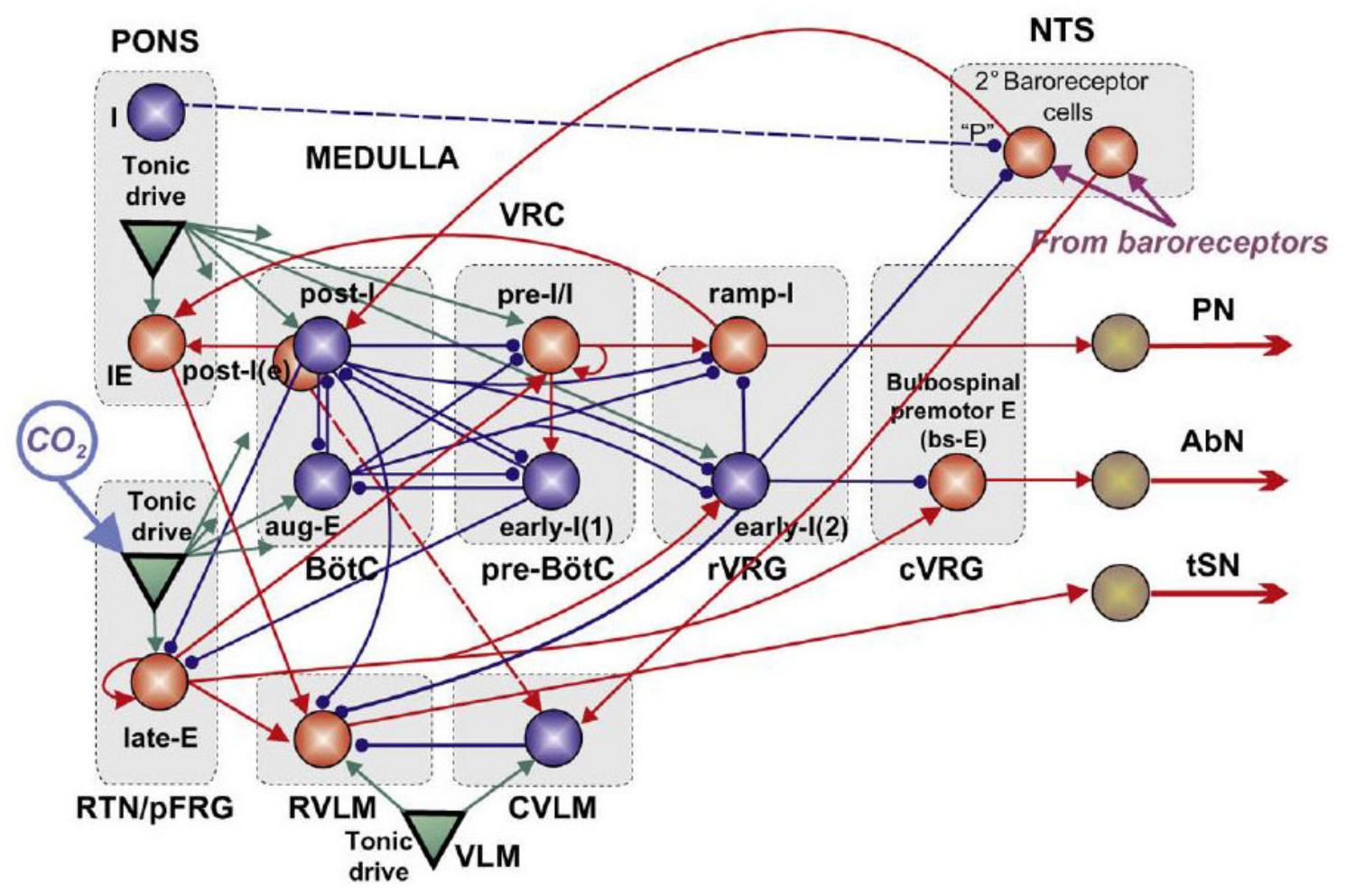

Fig. 3. Interactions amongst the breathing generator, and sympathetic oscillators in the brainstem. PreBötzinger complex preinspiratory inspiratory phase-spanning and decrementing early inspiratory units receive phasic inhibitory modulation from Bötzinger complex decrementing post-inspiratory and augmenting expiratory units and tonic excitatory drive from retrotrapezoid nucleus tonic units and parafacial respiratory group pre-inspiratory units, which receive the tonic excitatory drive from $\mathrm{CO}_{2}$-driven chemosensitive tonic units extant within the same nucleus. PreBötzinger complex preinspiratory inspiratory phase spanning units drive augmenting inspiratory units in the rostral division of the ventral respiratory group and decrementing early inspiratory units in the preBötzinger complex. recurrent Excitatory and inhibitory synaptic coupling synchronizes preBötzinger complex spontaneously bursting preinspiratory-inspiratory neuronal spiking. Pre-Bötzinger complex decrementing early inspiratory units convey inhibitory drive to Bötzinger complex augmenting late-expiratory units. Bötzinger complex postinspiratory and augmenting late-expiratory units exhibit reciprocal inhibition and convey inhibitory axodendritic and axosomatic drive to the caudal division of premotoneurons in the caudal ventral respiratory group. Bötzinger complex excitatory postinspiratory units drive metencephalic inspiratory-expiratory phase-spanning units. The firing of metencephalic inspiratory-expiratory phase-spanning units, tonic excitatory glutamatergic retrotrapezoid nucleus units, parafacial respiratory group late-expiratory unit drives and shapes rostral ventrolateral medullary presymapthetic bulbospinal neuronal spiking. Rostral ventrolateral medullary presympathetic bulbospinal neurons receive the inhibitory drive from Bötzinger complex postinspiratory units and rostral ventral respiratory group early inspiratory units and convey descending excitatory inputs to intermediolateral cell column preganglionic sympathetic neurons. Propriobulbar units distributed within the vast expanse of the ventral respiratory column nuclei provide the tonic excitatory drive to rostral ventrolateral medullary presymapthetic units and caudal ventrolateral medullary GABAergic units. Caudal ventrolateral medullary GABAergic propriobulbar interneurons convey a prominent and physiologically critical inhibitory modulation upon rostral ventrolateral medullary presympathetic units, the pharmacological elimination of which generates rapid and massive rises of dynamic sympathogenic neural efferent activity and arterial pressure magnitude. Rostral ventral respiratory group early inspiratory units convey inhibitory modulation upon co-extant rostral ventral respiratory group augmenting inspiratory premotoneurons, caudal ventral respiratory group expiratory premotoneurons, and rostral ventrolateral medullary presympathetic units. The metencephalon, raphé, retrotrapezoid nucleus, and ventrolateral myelencephalon constitute sources of tonic excitatory drive to respiratory-related and sympathetic units. Metencephalic inspiratory expiratory phase-spanning units receive the tonic excitatory drive from metencephalic propriobulbar units and Bötzinger complex excitatory postinspiratory units, dynamic spiking of which confers respirophasic modulation upon the rostral ventrolateral medullary presympathetic units. The phrenic motoneuronal and sympathetic axons constituting the phrenic nerve drive the principal mammalian inspiratory neuromotor discharge. The set of abdominal nerves drive expiratory neuromotor output. The thoracic sympathetic nerve represents a common, empirically accessible measure of sympathetic activity. The model contains two populations of nucleus tractus solitarius units receiving oscillatory baroreceptor inputs. One population of barosensitive nucleus tractus solitarius neurons synaptically excites caudal ventrolateral medullary GABAergic interneurons, and the complement population of barosensitive nucleus tractus solitarius units synaptically excites Bötzinger complex inhibitory decrementing postinspiratory units. Barosensitive nucleus tractus solitarius cells, in turn, receive prominent inhibitory modulation from metencephalic inspiratory units and rostral ventral respiratory group early inspiratory neurons. Excitatory (dark red) and inhibitory (purple) populations of units and interactions are indicated. RVLM, rostral ventrolateral medulla; CVLM, caudal ventrolateral medulla; VLM, ventrolateral medulla; RTN, retrotrapezoid nucleus; pFRG, parafacial respiratory group; late-E, late expiration; BötC, Bötzinger complex; pre-BötC, preBötzinger complex; rVRG, rostral ventral respiratory group; cVRG, caudal ventral respiratory group; bs-E, bulbospinal expiratory; PN, phrenic nerve; AbN, abdominal; tSN, thoracic sympathetic nerve; VRC, ventral respiratory column; I, inspiratory; IE, inspiratory expiratory phase spanning; late-E, expiratory; post-I, postinspiratory; pre-I/I, preinspiratory inspiratory phase spanning; early-I(1), preBötzinger early-inspiratory units; early-I(2), rVRG early inspiratory units. Modified with permission from Fig. 6 of Molkov et al. (2017). 


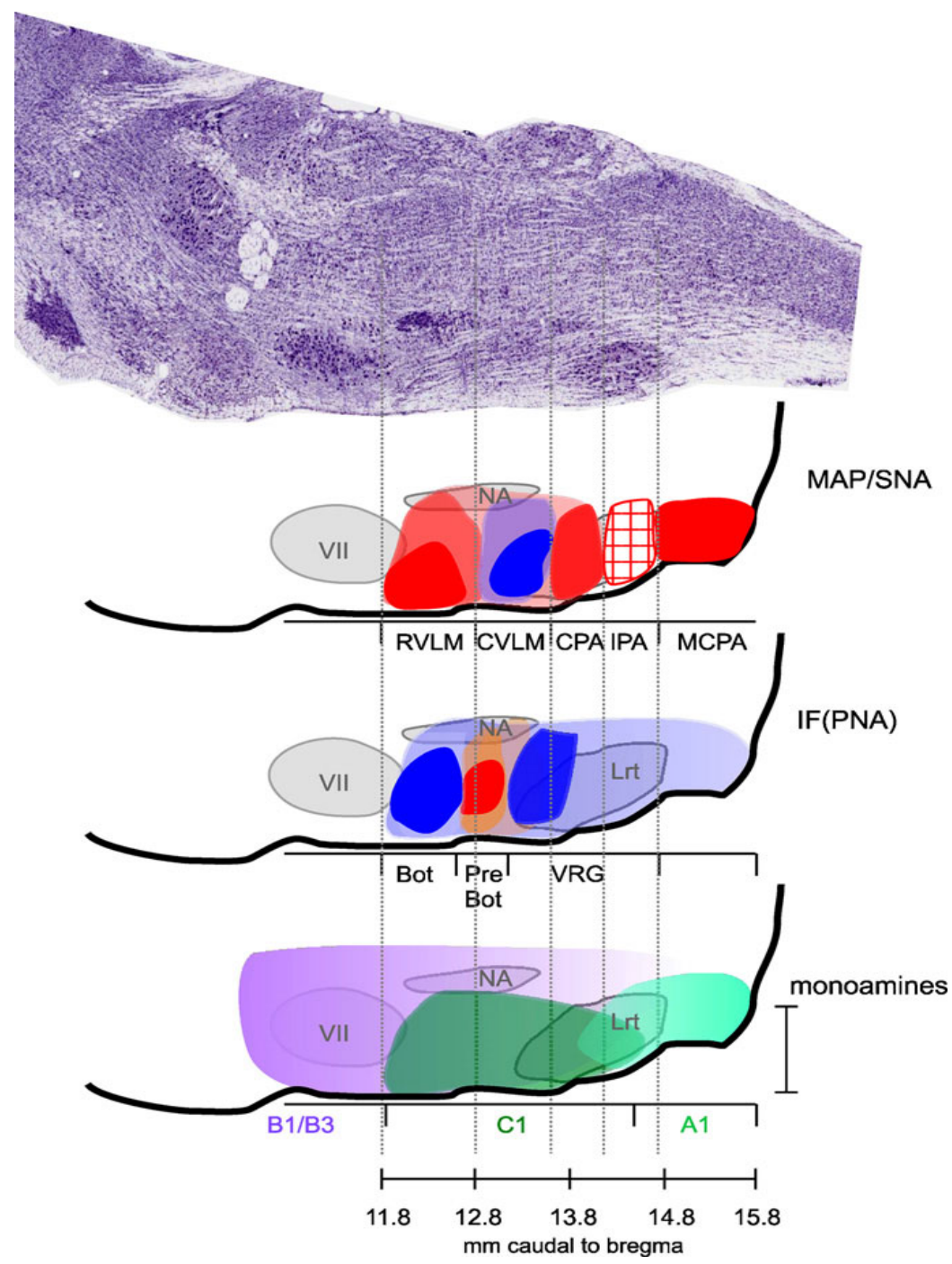

Fig. 4. Brainstem zones generating and modulating sympathetic oscillations and cardiovagal premotoneuronal spiking. Nissl stained parasagittal sections $1.8 \mathrm{~mm}$ lateral with respect to the midline. The chief medullary pre-sympathetic units project to intermediolateral cell column (IML) preganglionic sympathetic neurons reside within the rostral ventrolateral medulla (RVLM), rostral ventromedial medulla (RVMM), caudal raphé, ventrolateral metencephalic tegmentum (A5 catecholaminergic group of neurons), paraventricular nucleus of the hypothalamus, and the medullocervical pressor area (MCPA) spanning the caudal extent of the medulla and rostral extent of the cervical spinal cord. The MCPA extends at least as far caudal as C3/C4. The CVLM may be found caudally positioned with respect to the RVLM, constituted chiefly by GABAergic propriobulbar inhibitory interneurons (I-CVLM), which confer inhibitory modulation upon RVLM presympathetic units. Some I-CVLM units are modulated by the baroreflex arc and are consequently activated by higher-order barosensitive nucleus tratus solitarius (NTS) units, in turn, modulated by barosensitive afferents conveying oscillatory baroreceptor inputs from carotid sinus and aortic arch, relaying via cranial nerves IX and X, respectively. Another group of GABAergic I-CVLM units are baro-independent and tonically inhibit RVLM pre-sympathetic units. Some units in CVLM are glutamatergic (E-CVLM) and convey excitatory drive to RVLM presympathetic units. Chemoreceptor afferents terminate onto second-order neurons in the commissural nucleus of the NTS, which in turn project to and excite RVLM units directly. Chemosensitive commissural NTS units project to, excite, and sensitize RTN glutamatergic chemosensitive units. Prominent rises of arterial pressure were elicited by microinjecting glutamate into the commissural division of the nucleus tractus solitarius (unpublished observations). Cerebellectomy was not required. Careful dissection of the arachnoid bridging the interval between the calamus scriptorius and cerebellum failed to transgress parenchyma and successfully prepared the dorsal medullary surface for micropipette insertion. RTN units convey tonic excitatory drive to RVLM presympathetic units. Water undergoes a carbonic anhydrase-catalyzed chemical reaction to generate carbonic acid, which dissociates into bicarbonate anion and hydrogen cation. Hydrogen ions potently stimulate chemosensitive units within the retrotrapezoid nucleus, raphé, and nucleus tractus solitarius, among other central chemoreceptor sites. The caudal pressor area (CPA) may be found caudally positioned with respect to CVLM and mediates sympathoexcitation through the disinhibition of RVLM presympathetic units by inhibiting CVLM GABAergic inhibitory interneurons, and via facilitation, by activating a population of E-CVLM sympathoexcitatory glutamatergic units. Efferent interneuronal projections from the CPA to the commissural NTS may underlie the commissural NTS-dependence of pressor responses elicited by CPA stimulation. However, further studies are necessary to more fully elucidate the physiological significance of this specific pathway (Takakura et al., 2007). Yet to be determined, sources convey inhibitory modulation upon the CPA. RVLM-independence of sympathoexcitatory pressor responses elicited by chemical microstimulation effectively physiologically distinguishes the medullocervical pressor area from the caudal pressor area (Figs. 4) of Goodchild and Moon (2009). 
1997). Peripheral stimuli coordinately driving higher order nucleus tractus solitarius neurons or directly stimulated central chemoreceptors and interactions amongst propriobulbar interneuronal microcircuit oscillators emergently constituting neuronal ensembles generating breathing, sympathetic oscillations, and cardiovagal premotoneuronal spiking emergently generate sympathorespiratory coupling (Figs. 6-14) (Molkov et al., 2014).

Strengthening of autonomorespiratory coupling (sympathorespiratory $[\mathrm{SRM}]$ and respirosympathetic $[\mathrm{RSM}]$ modulation) by hypoxia and hypercapnia (Figs. 8-11) (Molkov et al., 2014; Moraes et al., 2012a,b,c; Zoccal, 2015; Zoccal and Machado, 2011) indicates peripheral effectors or central genesis mechanisms may alternately or coordinately conceivably mediate evident crossmodal modulation. Autonomorespiratory coupling persists despite decerebrative encephalotomy, vagotomy, and peripheral chemodenervation (Adrian et al., 1932; Marchenko et al., 2016; Zhou et al., 1996) supports and evidences the direct interaction of the central pattern generators (CPGs) in mediating crossmodal modulation of the respiratory, sympathetic, and parasympathetic activities. We use the term "autonomorespiratory" to efficiently indicate crossmodal modulation and coupling amongst and between the brainstem breathing generator, sympathetic oscillators, and dorsal medullary cardiovagal premotoneurons residing within the nucleus ambiguus and dorsal motor nucleus of the vagus. We thus operantly define autonomorespiratory to constitute the manifestation of, and the mechanisms emergently generating, sympathorespiratory, parasympathorespiratory, and sympathoparasympathetic crossmodal modulation and coupling across distinct though overlapping propriobulbar interneuronal microcircuit oscillators, nuclei, and reticular zones.

\section{Respirophasic modulation of rostral and caudal ventrolateral medullary neurons}

Myelencephalic nuclei chiefly constituted by propriobulbar interneurons and presympathetic bulbospinal neurons (Ghali, 2017a, 2018) exhibit respirophasic modulation, in rostral ventrolateral medullary (Fig. 5) (Baekey et al., 2010; Lipski et al., 1996a,b; McAllen, 1987; Miyawaki et al., 1995; Terui et al., 1986; Zoccal et al., 2009a,b, 2008), caudal ventrolateral medullary (Mandel and Schreihofer, 2006), caudal raphé (Gilbey et al., 1995), and ventrolateral metencephalic A5 catecholaminergic cell group neuronal recordings (McAllen, 1987; Molkov et al., 2017; Zoccal, 2015; Zoccal et al., 2008). respirophasic modulationPulmonary mechanosensitive nucleus tractus solitarius units modulate the discharge of presympathetic units modulating sinoatrial bursting frequency (Brodie and Russell, 1900; Daly, 1986). Rostral ventrolateral medullary neurons receive the tonic excitatory and inhibitory synaptic drive from propriobulbar interneuronal microcircuit oscillators (Frank et al., 2009; Gilbey et al., 1984; Guyenet and Koshiya, 1995; Guyenet, 2000; Mandel and Schreihofer, 2006; McAllen, 1986; Mendelowitz, 1999) and phasic oscillatory pulmonary mechanoreceptor, chemoreceptor, and baroreceptor synaptic inputs (Haselton and Guyenet, 1989; Lipski et al., 1996a,b; Rogers et al., 2000). Ventrolateral metencephalic A5 catecholaminergic cell group presympathetic bulbospinal units exhibit inspiratory, postinspiratory, or expiratory preferential modulation in anesthetized vagotomized rats (Gilbey et al., 1995). Respiratory propriobulbar interneuronal microcircuit oscillators may modulate sympathetic activity through direct synaptic interactions of overlapping propriobular interneuronal networks or indirectly by powerfully gat- ing baroreflex influences upon sympathetic oscillators and modifying baroreflex gain, sensitivity, and transfer kinetics (Eckberg and Orshan, 1977; Porta et al., 2015; Wallin and Eckberg, 1982). Respiratory gating of the baroreflex may confer respirophasic modulation upon sympathetic and parasympathetic oscillations by directly regulating dynamic arterial pressure magnitude and/or indirectly influencing sinoatrial action potential frequency (Figs. 13 and 14). Dynamic fluctuations of cardiac interval spectral bands may modulate arterial pressure spectral power (Julien, 2006). Persistence of sympathetic modulation of neural breathing evident in sinoaortic denervated preparations would seem to indicate sympathetic oscillations generated by medullary lateral tegmental field propriobulbar interneurons distributes and propagates to propriobulbar interneuronal microcircuit oscillators constituting the breathing pattern generator (Barman, 2020; Ghali, 2017a, 2018; Molkov et al., 2014). As an aside, acoustic tempo at 80 beats per minutes coordinately elicited increases in myocardial contraction frequency and spectral power of cardiac interval Traube-Hering and Mayer waves in human subjects (Watanabe et al., 2015), with cochleoautonomic coupling putatively organized by the medullary lateral tegmental field (Ghali, 2018).

Catecholaminergic and glutamatergic propriobulbar and spinally projecting bulbar presympathetic neurons constitute the rostral ventrolateral medulla, from which pressor responses may be elicited by electrical activation or glutamate microinjections (Figs. 4 and 5). Rostral ventrolateral medullary neurons exhibit respirophasic modulation of spiking frequency (Haselton and Guyenet, 1989; McAllen, 1987; Miyawaki et al., 1995; Terui et al., 1986), determined by extracellular or intracellular unitary recordings, and membrane voltage trajectories, determined exclusively by intracellular recordings (Lipski et al., 1996a,b). These patterns include earlyinspiratory, inspiratory, postinspiratory, and expiratory accentuation and typically phase-correlate with sympathetic neural efferent activity (i.e., cervical, thoracic, splanchnic, lumbar, renal nerves). McAllen (1987) revealed rostral ventrolateral medullary neurons exhibit peak unitary spiking frequency during the inspiratory epoch, with reciprocal postinspiratory attenuation of action potential frequency, in chloralose-anesthetized vagotomized cats. Haselton and Guyenet (1989) demonstrated inspiratory accentuation of rostral ventrolateral medullary neuronal spiking in halothane anesthetized rats. In contrast, Miyawaki et al. (1996) demonstrated inspiratorytroughs of rostral ventrolateral medullary neuronal spiking in anesthetized rats. Miyawaki et al. (1995) demonstrated respirophasic modulation of monomodally barosensitive rostral ventrolateral medullary neurons in anesthetized vagotomized rats, and Terui et al. (1986) demonstrated respirophasic modulation of coordinately barosensitive chemosensitive rostral ventrolateral medullary neurons in anesthetized vagotomized rabbits. Oscillatory baroreceptor inputs inhibit rostral ventrolateral medullary neuronal spiking frequency to a greater extent the inspiratory, compared with the expiratory, epoch, evidencing respiratory gating of baroinhibition of rostral ventrolateral medullary neurons, in vagotomized anesthetized rats (Miyawaki et al., 1995).

Overlapping Bötzinger complex and rostral ventrolateral medullary neuronal microcircuit oscillators constitute a chief node imposing respirophasic modulation upon sympathetic output (Moraes et al., 2012c; Sun et al., 1997), through which proximally-related nuclei (i.e., NTS, RTN) exert common coupling (Ghali, 2017a; Molkov et al., 2014). We conceive the rostral 


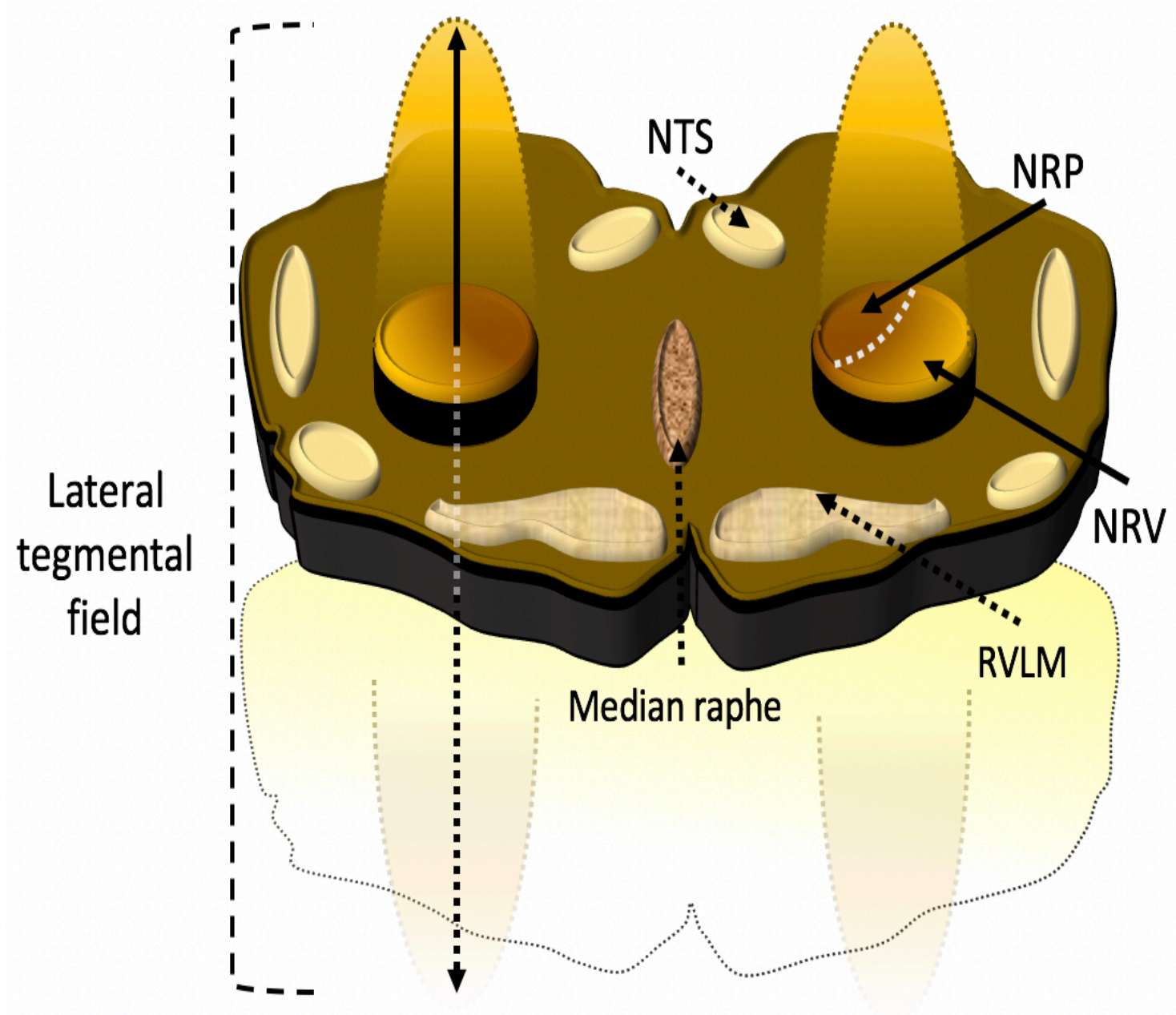

Fig. 5. Medullary lateral tegmental field and rostral ventrolateral medulla. The nucleus reticularis parvocellularis and nucleus reticularis oralis constitute the medullary division of the lateral tegmental field and span a vast extent of the reticular formation from the caudolateral medulla, becoming more compact with a rostromedial ascent towards the tip of the rostral ventrolateral medulla, giving the architectonic appearance of the cometary tail of the rostral ventrolateral medulla. The medullary lateral tegmental field contains barosensitive units conveying sympathoexcitatory and sympathoinhibitory propriobulbar synaptic inputs to rostral ventrolateral medullary bulbospinal inputs. Most neurophysiologists believe the medullary lateral tegmental field originates the sympathetic activity and conveys this to presympathetic bulbospinal units in nuclei residing within the metencephalon and myelencephalon, including the rostral ventrolateral medulla, rostral ventromedial medulla, caudal raphé, and ventrolateral metencephalic tegmentum. Medullary lateral tegmental field neuronal spiking precedes unitary activity of rostral ventrolateral medullary units and correlates with presympathetic bulbospinal neurons and cardiac sympathetic nerve discharge. Medullary lateral tegmental field propriobulbar interneuronal microcircuit oscillators modulate basal sympathoexcitation and mediate baroreceptor, chemoreceptor, Bezold-Jarisch, somatoautonomic, and visceroautonomic reflexes via NMDA and/or non-NMDA dependent glutamatergic fast excitatory synaptic neurotransmission. NRP, nucleus reticularis paragigantocellularis; NRV, nucleus reticularis ventralis; NTS, nucleus tractus solitarius; RVLM, rostral ventrolateral medulla.

ventrolateral medulla represents a compact specialized extension of the medullary lateral tegmental field supplying presympathetic bulbospinal drive to intermediolateral cell column preganglionic sympathetic neurons (Marchenko and Sapru, 2003; personal communication, V. Marchenko; see Ghali (2018). RVLM units receive the pre-presympathetic drive from the medullary division of the LTF. The coordinate drive of multiple sets of preganglionic sympathetic neurons residing in the intermediolateral cell column by RVLM and extra-RVLM supraspinal presympathetic units constitutes a mechanism emergently generating common coupling across heterologous and homologous pairs of sympathetic neural discharges. Most commonly, sympathetic activity exhibits postinspiratory or a phase-spanning late-inspiratory post-inspiratory accentuation of burst amplitude during normoxic or hyperoxic eucapnia. Mild, moderate, and severe degrees of hypercapnia, acute hypoxia, and chronic intermittent hypoxia incipiently shift this modulation to the late-expiratory phase and synchronously elicit late-expiratory abdominal nerve bursting (Figs. 8-11) (Molkov et al., 2017; Zoccal, 2015; Zoccal and Machado, 2011; Zoccal et al., 2008). Oscillatory synchrony amplifies the sympathetic activity. Interrogating the mechanistic underpinnings of this behavior informs emergent synchrony across disparate neural networks and fundamental strategies of neuro-computational processing.

Mandel and Schreihofer (2006) demonstrated varying patterns of respirophasic modulation imposed upon caudal ventrolateral medullary neurons in chloralose-anesthetized vagotomized 
rats (Mandel and Schreihofer, 2006). These included inspiratory augmentation, inspiratory inhibition, inspiratory augmentation with postinspiratory inhibition, and postinspiratory augmentation of respirophasic bursting (Mandel and Schreihofer, 2006). Baromodulated caudal ventrolateral medullary neurons (Mandel and Schreihofer, 2006) may constitute a chief mechanism conveying inhibitory modulation upon rostral ventrolateral medullary excitatory presympathetic neurons via GABAergic interneuronal projections respirophasic modulation (see Fig. 5). GABAergic caudal ventrolateral medullary units exhibiting postinspiratory preferential modulation may generate rostral ventrolateral medullary neuronal spiking troughs during the postinspiratory epoch. respirophasic modulation Electrical stimulation of aortic depressor nerve generates greater inhibitory effects upon rostral ventrolateral medullary neuronal spiking during the inspiratory, compared with the expiratory, epochs. Baro- and respiratory- modulated caudal ventrolateral medullary neurons exhibiting inspiratory preferential spiking analogously exhibit robust postinspiratory inhibition (Mandel and Schreihofer, 2006). Postinspiratory accentuations and troughs of rostral ventrolateral medullary neuronal spiking frequency may result from the influences of excitatory and inhibitory populations of Bötzinger complex decrementing postinspiratory cells (Sun et al., 1997) or caudal ventrolateral medullary postinspiratory modulated neurons (Mandel and Schreihofer, 2006), respectively. Occasional differential phase-specific respirophasic modulation of rostral ventrolateral medullary and caudal ventrolateral medullary neuronal spiking frequency suggests dynamic network reconfiguration of common propriobulbar interneuronal microcircuit oscillators coupling these zones (Mandel and Schreihofer, 2006).

\section{Respirophasic modulation of caudal raphé units}

Caudal raphé presympathetic neurons convey axodendritic and axosomatic drive to preganglionic sympathetic units residing in thoracolumbar intermediolateral cell column propriospinal interneuronal microcircuit oscillators driving preganglionic sympathetic neurons (Morrison and Gebber, 1982) and are modulated by respiratory-related propriobulbar interneuronal microcircuit oscillators residing within a few discrete zones. Respirophasic modulation of brainstem presympathetic units thence propagates throughout the sympathetic and parasympathetic neural networks (Gilbey et al., 1995; Lindsey et al., 1992). Gilbey et al. (1995) interrogated respirophasic modulation of caudal raphé presympathetic neurons in anesthetized vagotomized rats by conducting extracellular recordings of the cellular somata of raphé neurons. Antidromic activation conducted at upper thoracic spinal cord segments $\mathrm{T} 1$ to $\mathrm{T} 3$ electrophysiologically identified presympathetic units (Gilbey et al., 1995) in raphé pallidus, obscurus, and magnus (Gilbey et al., 1995), with a significant fraction of units exhibiting respirophasic modulation evidenced in a significant fraction of units (19 of 27 neurons). Neuronal modulatory patterns included inspiratory, expiratory, post-inspiratory, and biphasic early-inspiratory and post-inspiratory preferential discharge (Gilbey et al., 1995), recapitulating patterns of respirophasic modulation observed in rostral ventrolateral medullary presympathetic and caudal ventrolateral medullary GABAergic interneurons (Gilbey et al., 1995).

\section{Respirophasic modulation of preganglionic sympathetic neurons}

Preganglionic and postganglionic sympathetic neuronal spiking variably manifests inspiratory, expiratory, and/or postinspiratory patterns of modulation (Johnson and Gilbey, 1994; Zhou and Gilbey, 1992). In pentobarbital-anesthetized vagotomized rats, upper thoracic preganglionic sympathetic neurons exhibit a higher fraction of cells exhibiting respirophasic modulation compared with lower thoracic and lumbar preganglionic sympathetic neurons. These units exhibit predominantly postinspiratory patterns of modulation (Gilbey et al., 1986; Zhou and Gilbey, 1992). A rostrocaudal gradient in the magnitude of inspiratory and expiratory related respirophasic modulation of sympathetic activity exists along the length of the spinal cord, with differential convergence of inputs to the intermediolateral cell column preganglionic sympathetic neurons varying according to spinal segmental level. Respirophasic modulation of intermediolateral cell column preganglionic sympathetic neurons exhibits homology with unitary discharge patterns exhibited by the intercostal motoneurons supplying thoracic wall respiratory musculature (Gilbey et al., 1986). Cervical sympathetic preganglionic neurons exhibiting spontaneous spiking or activity elicitable by iontophoretically applied glutamate variably exhibit inspiratory or expiratory accentuation of burst amplitude, though some units remain non-modulated (Gilbey et al., 1986).

Spinal sympathoexcitatory neurons receiving convergent inputs from a specific zone within the rostral ventrolateral medulla characteristically span only a few spinal segments, consistent with the somatotopic organization of presympathetic neurons located within this region. Respirophasic modulation conferred upon intermediolateral cell column preganglionic sympathetic neurons likely derives from the composite influences of axodendritic and axosomatic synaptic inputs conveyed by presympathetic neurons residing within the rostral ventrolateral medulla (Baekey et al., 2010; Guyenet et al., 1990; Haselton and Guyenet, 1989; Lipski et al., 1996a,b; McAllen, 1987; Miyawaki et al., 1995; Terui et al., 1986; Zoccal et al., 2009a,b, 2008), caudal raphé (Gilbey et al., 1995), and ventrolateral metencephalic A5 catecholaminergic cell group, propriobulbar interneuronal networks, and somatic thoracic afferent fibers relaying proprioceptive information regarding chest wall muscle contraction. Thoracic wall non-respiratory-related afferents may confer inhibitory or excitatory modulatory synaptic drive upon intermediolateral cell column preganglionic sympathetic units.

Zhou and Gilbey (1992) conducted an elegant study investigating the respirophasic modulation of thoracolumbar myelic preganglionic sympathetic neurons in pentobarbital-anesthetized rats. Investigated perikarya were distributed throughout segments T13L2 of the ipsilateral intermediolateral cell chain (Zhou and Gilbey, 1992). Antidromic stimulation of the lumbar chain interposed between the $4^{\text {th }}$ and $5^{\text {th }}$ lumbar paravertebral ganglia electrophysiologically identified preganglionic sympathetic neurons (Zhou and Gilbey, 1992). Patterns of discharge of thoracolumbar preganglionic sympathetic neurons included inspiratory, early expiratory, and non-segmented expiratory preferential modulation, though a significant fraction of units lacked specific respirophasic modulation (Zhou and Gilbey, 1992). Glutamate iontophoresis elicited the resurgence of a quiescent population of neurons into readily evident neuronal spiking, with a similar proportion and distribution of active units exhibiting specific respirophasic modulation (Zhou and Gilbey, 1992). Preganglionic sympathetic neurons exhibiting 


\section{Control}

\section{$\mathrm{ClH}$}

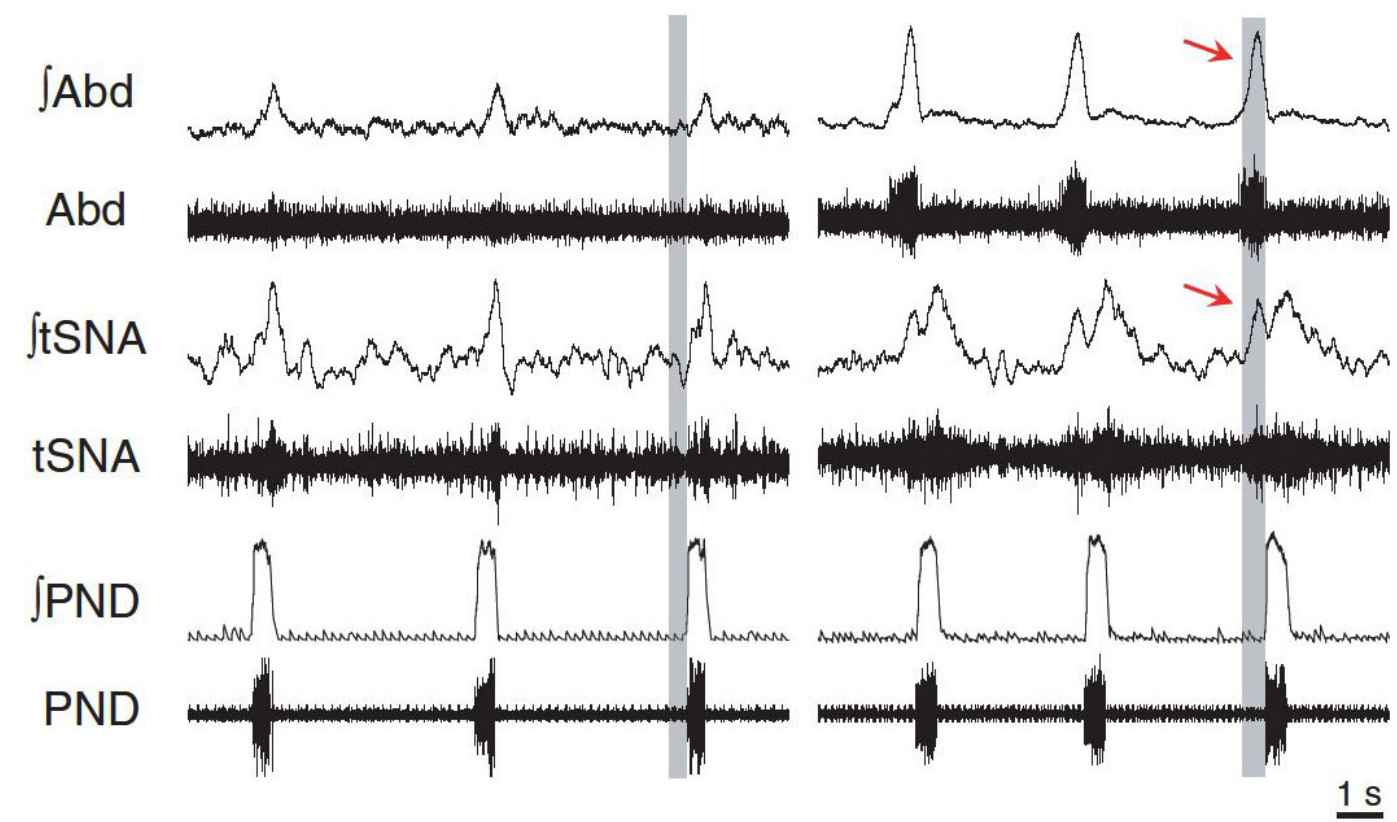

Fig. 6. Contemporaneously-obtained abdominal, thoracic sympathetic, and phrenic neurograms recordings in the soi-disant "working heart" brainstem preparation during normoxia and chronic intermittent hypoxic conditioning. Raw and integrated $\left(\int\right)$ activities of abdominal (Abd), thoracic sympathetic (tSNA), and phrenic nerve (PND) discharge during hyperoxic eucapnia (left panels) and chronic intermittent hypoxic-(CIH) conditioning (right panels). The shaded gray area in the neural recordings highlights the late expiratory phase of the respiratory cycle. Note obvious emergence and development of synchronous late-expiratory bursting in the neurograms of both the abdominal and thoracic sympathetic nerve activities (arrows) of rats conditioned with chronic intermittent hypoxia. Modified with permission from Fig. 2 of Zoccal et al. (2008).

respirophasic spiking coordinately demonstrated modulation varying with respect to the cardiac cycle (Zhou and Gilbey, 1992). Preganglionic sympathetic units are located at the T2 spinal level innervated neurons constituting the cervical sympathetic nerve (Zhou and Gilbey, 1992).

\section{Respirophasic modulation of sympathetic neural efferent activity}

Sympathetic neural efferent activity exhibits respirophasic modulation of rhythmic bursting (Fig. 6 and Figs. 8-14) (Koshiya and Guyenet, 1995; Mandel and Schreihofer, 2006; McAllen, 1987; Miyawaki et al., 1996; Seals et al., 1993; St. Croix et al., 1999). McAllen (1987) demonstrated inspiratory modulation of sympathetic neural activity in neurons of the subretrofacial nucleus (i.e., rostral ventrolateral medulla) and cervical sympathetic nerve in chloralose-anesthetized vagotomized cats (McAllen, 1987). Haselton and Guyenet (1989) later demonstrated various patterns of respirophasic modulation of lumbar sympathetic neural efferent activity, including inspiratory modulation, inspiratory inhibition, and early inspiratory inhibition, followed by postinspiratory modulation, in halothane anesthetized rats. Microinjections of the $\mathrm{GABA}_{A}$ receptor antagonist bicuculline into the rostral tip of the ventrolateral medulla, but not median raphe, coordinately enhanced phrenic nerve burst amplitude and frequency, lumbar sympathetic nerve burst amplitude and extent modulation by phrenic nerve activity, and dynamic mean arterial pressure magnitude and blunted the baroreflex in halothane-anesthetized vagotomized rats. However, microinjections of the glycine receptor antagonist strychnine and the $\mathrm{GABA}_{B}$ receptor antagonist phaclofen were with- out effect (Guyenet et al., 1990). Empirically demonstrated patterns of respirophasic modulation of sympathetic bursting have included exclusively postinspiratory accentuation of cervical and lumbar sympathetic nerve activity and preferentially inspiratory accentuation of cardiac, splanchnic, renal, adrenal, and muscle sympathetic nerve activity in vagotomized sinoaortic denervated halothane anesthetized rats (Numao et al., 1987; Seals et al., 1993; St. Croix et al., 1999), inspiratory accentuation of splanchnic sympathetic nerve discharge of vagotomized chloralose-anesthetized rats (Mandel and Schreihofer, 2006), inspiratory accentuation of inferior cardiac sympathetic nerve in chloralose-anesthetized vagotomized rats, and inspiratory and postinspiratory accentuation of splanchnic sympathetic nerve discharge in sinoaortic-denervated vagotomized urethane-anesthetized rats (Koshiya and Guyenet, 1995). These findings collectively indicate significant interstudy heterogeneity of patterns of respirophasic modulation of sympathetic oscillators.

Experimental interventions powerfully modifying respirophasic modulation of the sympathetic nerve discharge inform a thoughtful understanding of mechanisms emergently generating sympathorespiratory coupling (Miyawaki et al., 1996). Microinjections of specific N-methyl-D-aspartate (NMDA) (DL-2amino-5-phosphonovaleric acid), a-amino-3-hydroxy-5-methyl-4isoxazolepropionic acid (AMPA) (6-cyano-7-nitroquinoxaline-2,3dione), and kynurenic acid-type glutamate receptor antagonists into the rostral ventrolateral medulla abolish postinspiratory modulation of splanchnic sympathetic neural efferent activity in pentobarbital anesthetized rats, without modifying inspiratory modulation of efferent discharge persisted (Miyawaki et al., 1996). Postinspiratory modulation of lumbar sympathetic neural efferent activity may 
be augmented by Hypercapnia, and pharmacological antagonism of GABA, but not $\mathrm{GABA}_{B}$ or glycine, receptor modulated signaling in the rostral ventrolateral medulla abolished postinspiratory modulation of lumbar sympathetic neural efferent activity (Miyawaki et al., 1996). Postinspiratory modulation of lumbar sympathetic neural efferent activity decreased in parallel with dynamic increases of arterial pressure magnitude, indicating baroinhibition of respirophasic modulation of sympathetic neural efferent activity, and in response to microinjections of the nonselective glutamate receptor antagonist kynurenic acid, in halothane-anesthetized vagotomized rats. These findings corroborate fast excitatory glutamate receptor modulated signaling that contribute to sympathorespiratory modulation and contribute to tonic $\mathrm{GABA}_{A}$ and glycine-mediated fast inhibitory mechanisms in modulating coupling amongst the sympathetic and respiratory discharges (Miyawaki et al., 1996).

Respirophasic modulation of sympathetic efferent neural discharge exhibits heterogeneity across different preparation types (Haselton and Guyenet, 1989; Koshiya and Guyenet, 1995; Mandel and Schreihofer, 2006; McAllen, 1987; Miyawaki et al., 1996; Numao et al., 1987; Seals et al., 1993; St. Croix et al., 1999). Differences among patterns of respirophasic modulation across studies could putatively stem from differences in vagal continuity and whether animals are spontaneously-breathing versus mechanicallyventilated (Koshiya and Guyenet, 1995; Mandel and Schreihofer, 2006; McAllen, 1987; Numao et al., 1987; Seals et al., 1993; St. Croix et al., 1999), powerfully influencing discharge patterns and properties of slow and fast adapting pulmonary stretch receptors and imposition of these oscillatory inputs upon nucleus tractus solitarius units (Fenik, 1992; Preobrazhenskiı̌ et al., 1989). Respirophasic modulation of sympathetic neural efferent activity may alternately arise from the overlap of, and interaction between, Bötzinger complex propriobulbar interneuronal decrementing postinspiratory and augmenting late-expiratory units with rostral ventrolateral medullary presympathetic cells (Moraes et al., 2014a) or pulmonary stretch receptor influences upon sympathetic oscillators. Bötzinger complex decrementing post-inspiratory units driving respirophasic modulation of sympathetic oscillations may natively entrain this coupling, or be driven by, pulmonary stretch receptor mechanosensitive (Preobrazhenskil et al., 1989) or barosensitive nucleus tractus solitarius units (Fig. 5) (Rogers et al., 2000).

\section{Mechanisms underlying crossmodal coupling across neural breathing and sympathetic oscillations \\ 7.1 Overview}

Mechanisms contributing to crossmodal modulation among neural breathing (Moraes et al., 2012a,b,c; Zoccal, 2015), supraspinal and spinal sympathetic-related neuronal and sympathetic neural efferent oscillations (Lipski et al., 1996a,b; McAllen, 1987; Miyawaki et al., 1996) (Fig. 6-9 and Fig. 12-14), and cardiovagal premotoneuronal spiking (Figs. 10 and 11) (Bishop, 1974; Lindsey et al., 1998) utilize coordinate bulbar and peripheral inputs to, and direct interaction amongst, overlapping populations of propriobulbar interneuronal microcircuit oscillators constituting the generators incipiently giving rise to evident activities (Figs. 1-5) (Ghali, 2020; Guyenet et al., 2019; Marchenko et al., 2016; Moraes et al., 2012c; Sun et al., 1997). Sympathorespiratory coupling utilizes mechanisms organized chiefly and principally by, and predicated upon intact functionality of, the medullary lateral tegmental field (Fig. 4) (Ghali, 2017a, 2018), Bötzinger complex (Molkov et al., 2014; Moraes et al., 2012c; Sun et al., 1997; Zoccal et al., 2008), rostral ventrolateral medulla (RVLM) (Haselton and Guyenet, 1989; Lipski et al., 1996a,b; McAllen, 1987; Miyawaki et al., 1996, 1995; Moraes et al., 2014a,b), caudal ventrolateral medulla (CVLM) (Mandel and Schreihofer, 2006), retrotrapezoid nucleus (RTN) and parafacial respiratory group (pFRG) (Fig. 5) (Abdala et al., 2009; Molkov et al., 2017, 2014; Moraes et al., 2012a) dorsolateral (Baekey et al., 2010; Hayward et al., 2004) and ventrolateral metencephalic tegmentum (Dawid Milner et al., 2003), mesencephalic colliculi (Ghali, 2020; ligaya et al., 2012; Martin and Booker, 1878; Subramanian and Holstege, 2014) and periaqueductal gray matter (PAG) (Iigaya et al., 2010; Martin and Booker, 1878; Subramanian and Holstege, 2014), and dorsomedial hypothalamus (DMH) (Horiuchi et al., 2009) and paraventricular nucleus (Koba et al., 2018). The mesencephalic colliculi (ligaya et al., 2012; Martin and Booker, 1878 ) and dorsomedial and dorsolateral divisions of the periaqueductal gray matter (Ghali (2020) mesencephalon reference; Subramanian and Holstege (2014)) coordinately modulate breathing rate and depth, sympathetic neural efferent activity, eliciting prominent increases of dynamic arterial pressure magnitude and myocardial contraction frequency, hyperpnea, and behavioral defense responses.

Direct interaction amongst the propriobulbar interneuronal microcircuitry underlying neurogenesis of breathing, sympathetic oscillations, and parasympathetic cardiovagal premotoneuronal spiking may modulate the described network mechanisms (Fig. 5) (Molkov et al., 2017). BötzC propriobulbar expiratory interneurons overlap with rostral ventrolateral medullary presympathetic units (Guyenet et al., 1990; Moraes et al., 2012c; Sun et al., 1997), likely representing a critical myelencephalic node subjectable to coordinate modulation of breathing rhythm and amplitude and respirophasic modulation of sympathetic bursts. Excitatory synaptic drive deriving from chemosensitive retrotrapezoid nucleus units or chemoreceptors relaying through nucleus tractus solitarius units shifts the bulk of sympathetic burst spectral power towards the expiratory epoch (Molkov et al., 2011; Sun et al., 1997), perhaps offsetting expiratory-related reductions of preload by generating venoconstriction and enhancing microcirculatory tissue perfusion during troughs of oscillatory oxygen tension, a set of conjectures subjectable to empirical validation. Experimental excitatory chemical microstimulation of hypothalamic, Kölliker-Fuse, and medial parabrachial (Dutschmann and Herbert, 2006; Mörschel and Dutschmann, 2009), ventrolateral metencephalic (Dawid Milner et al., 2003), vestibular, and reticulospinal zones coordinately modulate breathing, sympathetic oscillations, and cardiovagal premotoneuronal spiking. Complexly organized brainstem neuronal arrays of bulbospinal and propriobular reticular units (Anastasievich et al., 1974; Kostiuk and Preobrazhenskiū, 1966; Kostiuk and Preobrazhenskii, 1967) monosynaptically or polysynaptically receiving afferent inputs may prominently modulate coupling amongst and within somatovisceral, respiratory, and sympathetic neural networks (D'iachenko and Preobrazhenskiǐ, 1991). Vocal cord stimulation potently enhances gigantocellular tegmental field descending medullospinal neuronal spiking frequency in Nembutalanesthetized cats (D'iachenko and Preobrazhenskiǔ, 1991). The described pathways constitute critical mechanisms by which neural networks emergently generate soi-disant vaso-cardio-respiratory coupling. 
$A a$
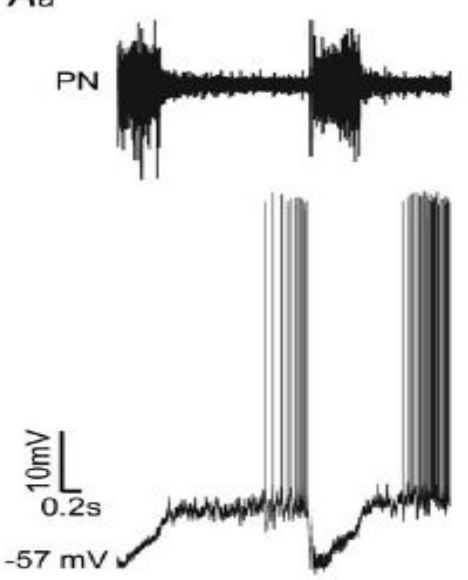

$B a$

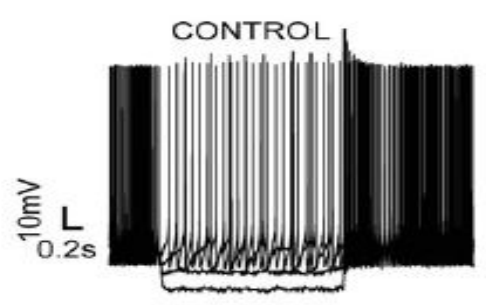

Ca

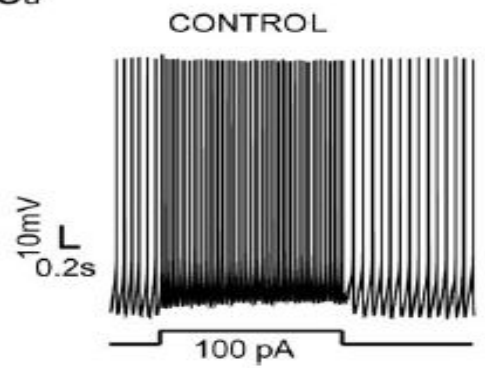

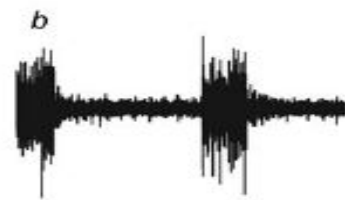
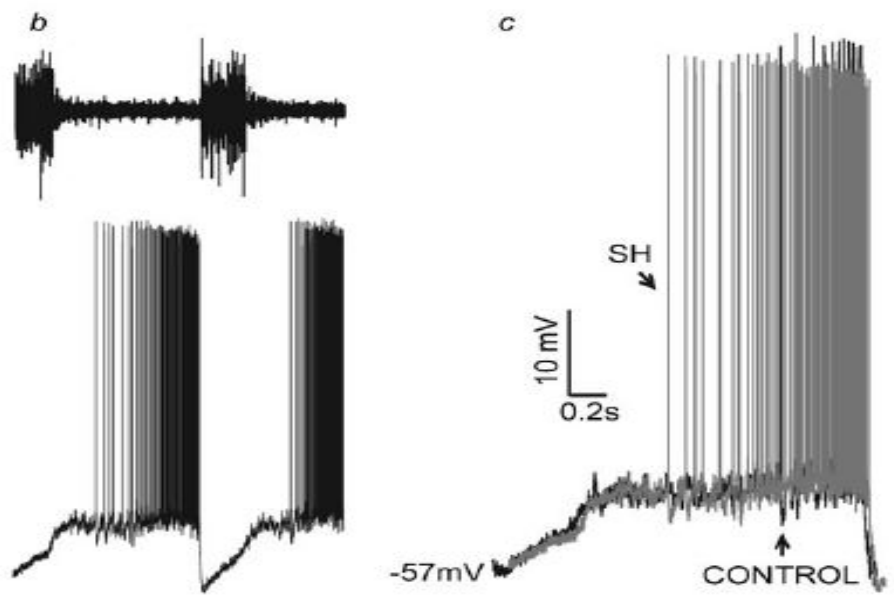

b
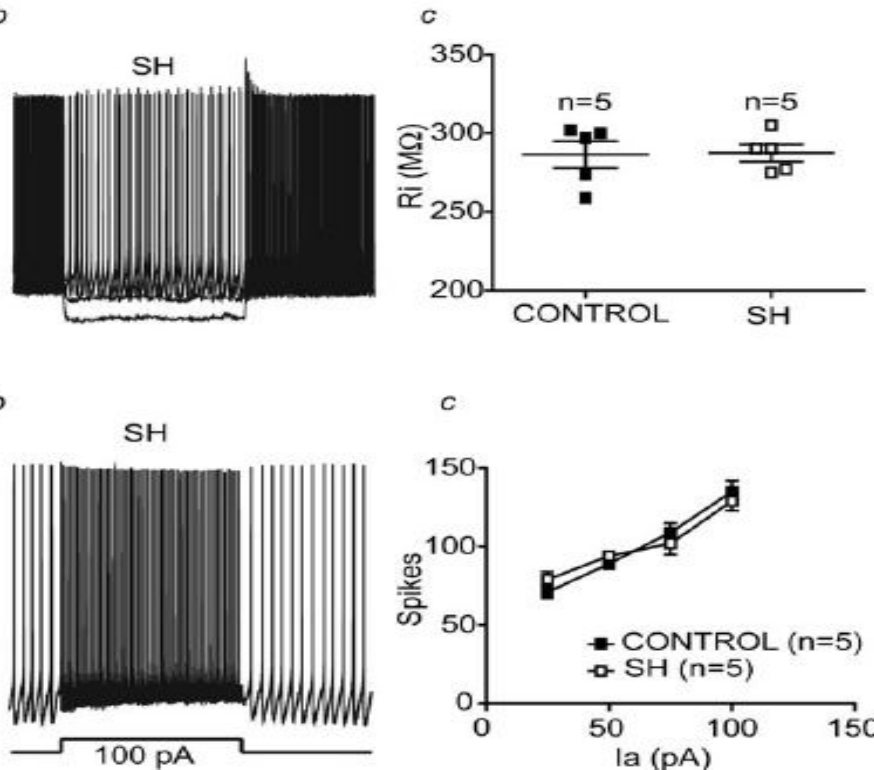

c

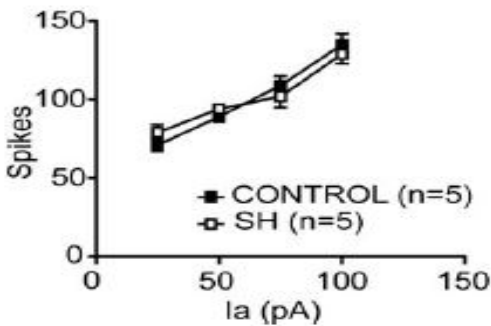

Fig. 7. Intracellular recordings of Bötzinger complex augmenting late-expiratory neurons. A: Phrenic neurogram and intracellular recordings of Bötzinger complex augmenting expiratory units reveal oscillations of membrane voltage with augmenting action potential spiking frequency during the late expiratory phase in normotensive $(A a)$ and spontaneously hypertensive $(A b)$ rats, Superimposition of Bötzinger complex augmenting late-expiratory intracellular recordings in normotensive and spontaneously hypertensive rats demonstrates phase-advance of neuronal spiking earlier into expiration ( $A c$ ), putatively implying Botzinger complex postinspiratory units may reciprocally exhibit less amplitude in the hypertensive condition. B: Antagonism of fast inhibitory synaptic neurotransmission failed to differentially modify neuronal input resistance in normotensive $(B a)$ or spontaneously hypertensive ( $B b$ ) rats, graphically depicted in $(B c)$. Delivery of electrical pulses in current-clamp mode ranging from 25 to 100 picoamperes enhanced Bötzinger complex augmenting late-expiratory neuronal spiking frequency in normotensive $(\mathrm{Ca})$ and spontaneously hypertensive $(\mathrm{Cb})$ rats. However, the two groups failed to exhibit differences in neuronal spiking-current density slope $(C c)$. Visual inspection of panels $B a, B b, C a$, and $C b$ appear to evidence somewhat regular oscillations of neuronal spiking frequency (alternating thick and thin spike clusters) $\sim 1.7 \mathrm{~Hz}$, perhaps representing slow sympathetic oscillations distributing to Bötzinger complex neurons. Spike height also exhibits prominent oscillations in panels $B a$ and $B b$. Ia, current; M $\Omega$, megaohms, mV, microvolts; pA, picoamperes; Ri, internal resistance; $\mathrm{SH}$, spontaneously hypertensive. Modified with permission from Fig. 4 of Moraes et al. (2014a).

Brainstem propriobulbar and myelic propriospinal interneuronal microcircuit oscillators generating sympathetic oscillations (Barman, 2020; Ghali, 2018; Guyenet, 2006) receive prominent modulatory influences deriving from oscillatory vagal baroreceptors (Rogers et al., 2000), chemoreceptors (Guyenet et al., 2019), and pulmonary stretch receptors (Breuer, 1868; Hering, 1868; Luck, 1969; Moore, 1927) conveyed through nucleus tractus solitarius units (Fig. 5) and respiratory-related neurons (Guyenet, 2006; Molkov et al., 2017, 2014; Moraes et al., 2014a; Rogers et al., 2000).
Neural respiratory propriobulbar and bulbospinal premotoneuronal, sympathetic propriobulbar, presympathetic bulbospinal, and preganglionic sympathetic, and cardiovagal preganglionic neurons receive mutually convergent inputs conveyed monosynaptically and polysynaptically from coordinate higher-order neuronal ensembles and peripheral afferent inputs, providing common coupling mechanisms organizing the direct interaction of the central pattern generators (Fig. 5) (Baekey et al., 2010; Ghali, 2017a, 2018; Molkov et al., 2017). We provide a few examples of sympathorespiratory 
modulation and coupling in the brainstem and spinal cord neurons and sympathetic efferent neural discharge (Figs. 5 and 14) (Zoccal, 2015).

\subsection{Kölliker-fuse and medial parabrachial nuclei}

Neurons residing within the Kölliker-Fuse and medial parabrachial nuclei constituting a significant bulk of the dorsolateral metencephalic tegmentum (Dutschmann and Herbert, 2006; Mörschel and Dutschmann, 2009) generate inspiratory off switching via the provision of a tonic excitatory glutamatergic drive to glycinergic Bötzinger complex decrementing post-inspiratory neurons (Fig. 5) (Dutschmann and Herbert, 2006; Mörschel and Dutschmann, 2009; Smith et al., 2009). These cells powerfully modulate the discharge of rostral ventrolateral medullary presympathetic units and cardiovagal premotoneurons residing within the dorsal motor nucleus of the vagus and nucleus ambiguus (Baekey et al., 2010; Molkov et al., 2014). Eliminating respirophasic modulation of sympathetic oscillations by transectively interrupting pontomedullary continuity (Molkov et al., 2014) supports a requisite contribution of Kölliker-Fuse and medial parabrachial nuclei glutamatergic neurons in crossmodally modifying and rhythmically entraining sympathetic output (Baekey et al., 2010). Metencephalogenic sympathorespiratory modulation may be conveyed via direct monosynaptic phasic inputs deriving from Kölliker-Fuse and medial parabrachial neurons to rostral ventrolateral medullary cells (Fig. 5) (Dutschmann and Herbert, 2006; Mörschel and Dutschmann, 2009), or through polysynaptic propriobulbar interactions relaying through neuronal ensembles extant within the medullary ventral respiratory column nuclei (Molkov et al., 2014). The metencephalon imposes modulatory influences upon rostral ventrolateral medullary presympathetic neurons via the provision of a tonic excitatory glutamatergic drive to Bötzinger complex decrementing postinspiratory neurons and through the provision of tonic excitatory glutamatergic drive conveyed by inspiratory-expiratory phase-spanning neurons (Fig. 5) (Baekey et al., 2010; Farmer et al., 2016). Metencephalic units, directly and indirectly, provide coordinate phasic and/or tonic excitatory drive to rostral ventrolateral medullary presympathetic units and Bötzinger complex expiratory cells, emergently generating the evident crossmodal modulation typifying sympathorespiratory coupling (Fig. 5) (Baekey et al., 2010; Molkov et al., 2014).

\subsection{Retrotrapezoid nucleus}

Homogenously phox $2 \mathrm{~b}$ transcription factor-expressing glutamatergic propriobulbar interneurons exhibiting exquisite chemosensitivity to arterial and neural interstitial carbon dioxide tension and hydrogen ion concentration constitute the retrotrapezoid nucleus (Guyenet et al., 2019). Glutamatergic retrotrapezoid nucleus units convey tonic excitatory glutamatergic drive to ventrolateral medullary respiratory nuclei and sympathetic oscillators (Figs. 1 and 5) (Guyenet et al., 2019; Molkov et al., 2014). These cells overlap with a cluster of parafacial respiratory group pre-inspiratory modulated units, which may convey synaptic drive to the preBötzinger complex pre-inspiratory driver population. The overlapping parafacial respiratory group oscillator contains rhythmically and spontaneously bursting neurons (Onimaru et al., 2018) exhibiting preinspiratory discharge (Onimaru et al., 2006). These pFRG pre-I units project to, and may putatively drive, the activity of preBötzinger complex preinspiratory neurons and Bötzinger complex expiratory abdominal premotoneurons in Bötzinger com- plex (Molkov et al., 2017), as well as rostral ventrolateral medullary cells. The zone represents an auxiliary expiratory rhythm generator according to studies conducted by Janczewski and Feldman (2006) in the brainstem-spinal cord preparation of the juvenile rat in situ. It constitutes an opioid-insensitive inspiratory rhythm generator operant within the incipient minutes of post-womb life. Vaginalbirth-mediated generation of opiates inhibits preBotzinger complex preinspiratory neuronal spiking, though parafacial respiratory group units remain refractory (Feldman and Del Negro, 2006). The retrotrapezoid nucleus and parafacial respiratory group complex likely constitute a region mediating and organizing common crossmodal coupling of breathing, sympathetic oscillations, and cardiovagal premotoneuronal spiking through the coordinate provision of excitatory drive to rostral ventrolateral medullary and Bötzinger complex cells (Abdala et al., 2009; Moraes et al., 2014a, 2012a), effectively generating coupled and synchronous late-expiratory bursting in abdominal and thoracic sympathetic neural efferent activities (Abdala et al., 2009; Molkov et al., 2017; Moraes et al., 2012a; Zoccal, 2015). Synaptic neuroplasticity of retrotrapezoid nucleus and parafacial respiratory group units may be critically implicated in strengthening sympathorespiratory crossmodal modulation in rats subjected to hypercapnia and acute and chronic intermittent hypoxia (Molkov et al., 2017; Zoccal, 2015)., the pathogenesis of neurogenic arterial hypertension, and sustained coordinate synchronous late-expiratory bursting in sympathetic and thoracic sympathetic nerve activity persisting at rest in response to hypercapnia and chronic intermittent hypoxic conditioning (Molkov et al., 2014, 2011; Moraes et al., 2013; Zoccal et al., 2008).

\subsection{Bötzinger complex}

Chiefly glycinergic, decrementing post-inspiratory (Ezure and Manabe, 1988) and, principally GABAergic, augmenting late expiratory (Jiang and Lipski, 1990) propriobulbar interneurons (Fig. 5), exhibiting mutual and reciprocal inhibition (see models by Molkov et al. (2017) and Smith et al. (2007, 2009)) constitute the Bötzinger complex, Bötzinger complex decrementing postinspiratory and augmenting late-expiratory neurons convey inhibitory synaptic drive upon preBötzinger complex preinspiratory (pre-I), preinspiratory-inspiratory (pre-I/I) phase-spanning, and decrementing early-inspiratory (dec early-I) neuronal somatodendritic membranes (Molkov et al., 2017) and excitatory drive to caudal ventral respiratory group (cVRG) expiratory premotoneurons (Smith et al., 2009). A population of GABAergic Bötzinger complex bulbophrenic units conveys supraspinal descending inhibitory modulation upon inspiratory-related phrenic motoneuronal somatodendritic membranes (Figs. 1 and 2). Bötzinger complex augmenting late expiratory neuronal spiking (Fig. 7) generates correlated late expiratory bursting in abdominal and thoracic sympathetic nerve discharge in response to eucapnic hypoxia and hypercapnia (Figs. 811) (Moraes et al., 2014a). Chronic intermittent hypoxia could increase the activity of Bötzinger complex augmenting late expiratory neuronal spiking (Fortuna et al., 2008; Janczewski and Feldman, 2006) via increased excitability of these units or by reciprocal reductions of Bötzinger decrementing postinspiratory neurons (Ezure and Manabe, 1988; Molkov et al., 2017; Smith et al., 2009), amplifying augmenting late-expiratory activity via disinhibition, conjectures supported by interactions amongst coupled distinct propriobulbar interneuronal microcircuit oscillators generating the breathing 
rhythm and pattern (Molkov et al., 2017).

Bötzinger complex decrementing postinspiratory units receive excitatory glutamatergic synaptic inputs from neurons residing within the Kölliker-Fuse and medial parabrachial nuclei (Fig. 5) (Dutschmann and Herbert, 2006; Mörschel and Dutschmann, 2009), barosensitive nucleus tractus solitarius neurons (Miyazaki et al., 1999), and nucleus tractus solitarius pump cells conveying spatiotemporally dynamic alveolar wall and septal stretch (Marchenko and Sapru, 2000) PreBötzinger complex augmenting pre-inspiratory, augmenting pre-inspiratory inspiratory phasespanning, and decrementing early-inspiratory neurons and rostral ventral respiratory group decrementing early inspiratory neurons (Molkov et al., 2017; Smith et al., 2007) dynamically impose inhibitory modulation upon Bötzinger complex decrementing postinspiratory units. The sequential activity of ventrolateral medullary late-inspiratory neurons and Bötzinger complex decrementing postinspiratory units terminate the inspiratory epoch (Cohen et al., 1993; Okazaki et al., 2001) and the post-inspiratory component of the cervical vagus and recurrent laryngeal nerve discharge (see Bruce (1988) and Marchenko et al. (2016)). Bötzinger complex decrementing postinspiratory units modulate spatiotemporally dynamic sympathetic propriobulbar (see Moraes et al. (2012c) and Sun et al. (1997)) and cardiovagal premotoneuronal (see Gilbey et al. (1984)) neuronal spiking.

Overlap of rostral ventrolateral medullary presympathetic neurons with inspiratory and Bötzinger complex expiratory propriobulbar interneurons and inspiratory propriobulbar interneurons within ventral respiratory column nuclei (see Moraes et al. (2012c) and Sun et al. (1997)) may generate tightly-coupled sympathorespiratory modulation (Mandel and Schreihofer, 2006; McAllen, 1987; Molkov et al., 2014; Moraes et al., 2012a,b,c; Zoccal, 2015; Zoccal and Machado, 2011; Zoccal et al., 2008). Bötzinger complex propriobulbar and rostral ventrolateral medullary propriobulbar and presympathetic bulbospinal neurons are intermingled and make direct synaptic contacts (see Moraes et al. (2012c) and Sun et al. (1997)). Increased activity among either of these neuronal ensembles, though functionally distinct, could effectively amplify and augment the activity of the other group (Molkov et al., 2014). Specifically, rostral ventrolateral medullary neurons receive inhibitory modulation by Bötzinger complex decrementing post-inspiratory units during early expiration (Baekey et al., 2010). Postinspiratory and inspiratory neurons may thus effectively modulate rostral ventrolateral medullary unitary activity via direct monosynaptic projections or indirectly via relays through caudal ventrolateral medullary GABAergic propriobulbar interneurons (Zoccal et al., 2008). Rostral ventrolateral medullary neurons exhibit peak spiking frequency during the inspiratory epoch and trough spiking frequency the during the postinspiratory epoch during rest (Haselton and Guyenet, 1989), a pattern of modulation putatively generated by inhibitory axodendritic and/or axosomatic drive deriving from Bötzinger complex decrementing postinspiratory units (see Molkov et al. (2014) and Sun et al. (1997)). Differential modulation patterns of rostral ventrolateral medullary propriobulbar and presympathetic units vary across the animal model, preparation type, and experimental conditions (Lipski et al., 1996a,b; McAllen, 1987; Miyawaki et al., 1996) may result from corresponding statedependent reconfiguration of propriobulbar interneuronal microcircuit oscillators constituting the neural breathing and myelencephalic sympathetic (Zoccal, 2015).
Synaptic neuroplasticity may coordinately amplify the discharge of these nuclei in response to hypercapnia and acute and chronic intermittent hypoxia (Molkov et al., 2014). Excitatory glutamate microstimulatory activation of Bötzinger complex neurons in the ventrolateral medulla powerfully reduces the amplitude of phrenic nerve discharge. It augments dynamic arterial pressure magnitude in isoflurane-anesthetized adult and unanesthetized decerebrate juvenile rats (Marchenko et al., 2016). Conversely, muscimol inhibition of Bötzinger complex, preBötzinger complex, and/or rostral ventral respiratory group units abolishes sympathorespiratory coupling, experimental findings indicating entrainment amongst expiratory and sympathetic oscillators (Koshiya and Guyenet, 1996). Accordingly, emergent generation of coordinate late-expiratory bursting in abdominal and thoracic sympathetic neural efferent activity in response to hypoxia and hypercapnia (Figs. 8-11) (Molkov et al., 2014; Zoccal and Machado, 2011; Zoccal et al., 2008) may be mediated and organized by direct interaction amongst and between Bötzinger complex and rostral ventrolateral medullary propriobulbar interneuronal oscillators (Fig. 5) (Molkov et al., 2014; Moraes et al., 2012a,b,c; Zoccal and Machado, 2011; Zoccal et al., 2008).

\subsection{Rostral ventrolateral medulla}

Direct interaction of the respiratory, central pattern generator and sympathetic oscillators constitutes an elegant mechanism mediating sympathorespiratory coupling (Molkov et al., 2014; Moraes et al., 2012c; Sun et al., 1997). Interactions amongst propriobulbar and bulbospinal neuronal ensembles residing within the overlapping rostral ventrolateral medulla and Bötzinger complex (Fig. 3) (see Paxinos et al. (1985) and Sun et al. (1997)) may couple presympathetic and expiratory units and emergently generate inspiratory, postinspiratory, and late-expiratory patterns of modulation conveyed to sympathetic oscillations (Figs. 6-9 and Figs. 12-14) (Moraes et al., 2014a, 2012a, 2014b, 2012b,c; Zoccal et al., 2008). Overlap of caudal ventrolateral medullary GABAergic interneurons with preBötzinger complex augmenting pre-I, augmenting pre-I/I, and decrementing early-I units and rostrally extant rostral ventral respiratory group augmenting inspiratory neurons (Fig. 3) (see Goodchild and Moon (2009)), as well as caudal ventrolateral medullary GABAergic propriobulbar interneuronal synaptic drive conveyed to rostral ventrolateral medullary presympathetic units, may impose respirophasic modulation upon sympathetic oscillations (Mandel and Schreihofer, 2006) and autonomic modulation upon a respiratory-related activity (see Baekey et al. (2010)). Coordinate excitatory synaptic drive conveyed by the glutamatergic retrotrapezoid nucleus and parafacial respiratory group neurons to Bötzinger complex expiratory neurons and rostral ventrolateral medullary presympathetic cells (Guyenet et al., 2019), as well as direct interaction of overlapping propriobulbar excitatory and inhibitory neuronal ensembles of the Bötzinger complex and rostral ventrolateral medulla (Moraes et al., 2012c; Sun et al., 1997), may constitute robust mechanisms contributing prominently and mediating, sympathorespiratory modulation at rest and hypoxia and hypercapnia-mediated generation and strengthening of sympathorespiratory coupling (Fig. 5) (Molkov et al., 2014). Excitatory glutamate stimulatory activation of ventral respiratory column nuclei occasionally elicits coordinate enhancement or attenuation of dynamic arterial pressure magnitude (Marchenko et al., 2016), reflecting intimate intermingling of the propriobulbar interneuronal microcircuit oscillators generating the breathing rhythm 
A

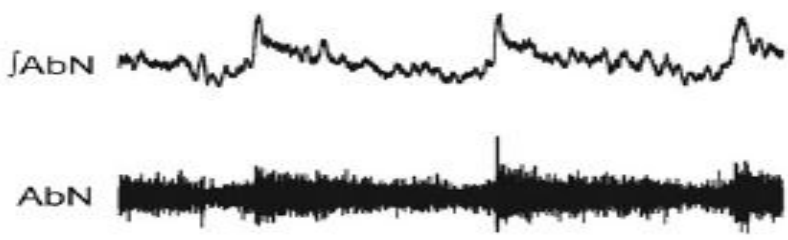

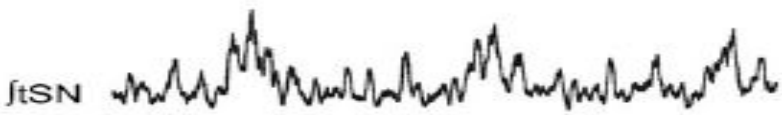

tSN

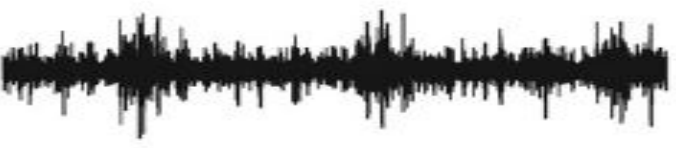

$P N$

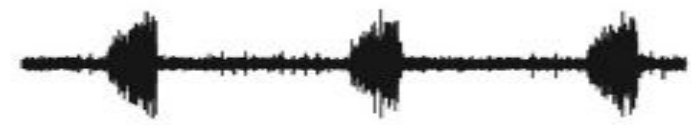

B

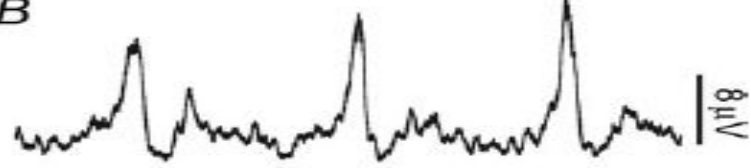
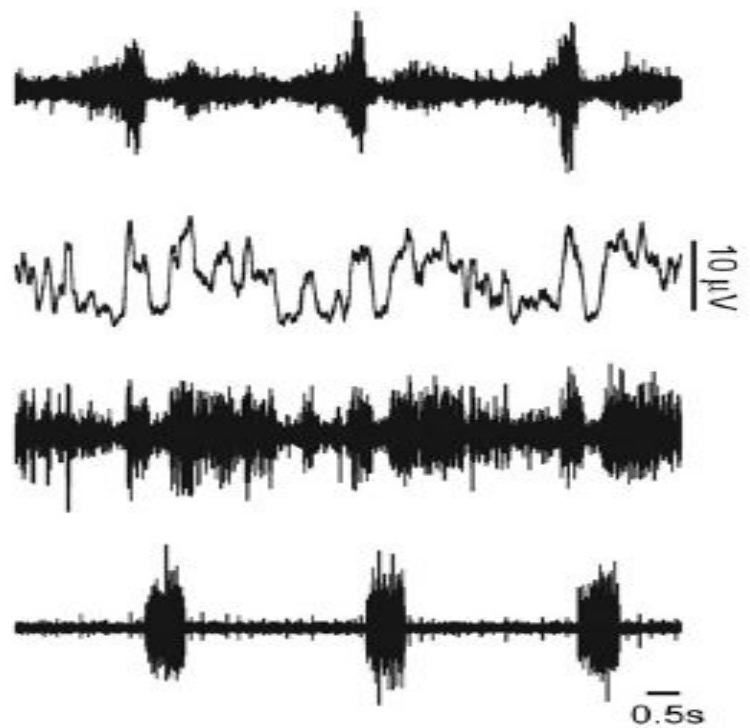

E

C

$D$

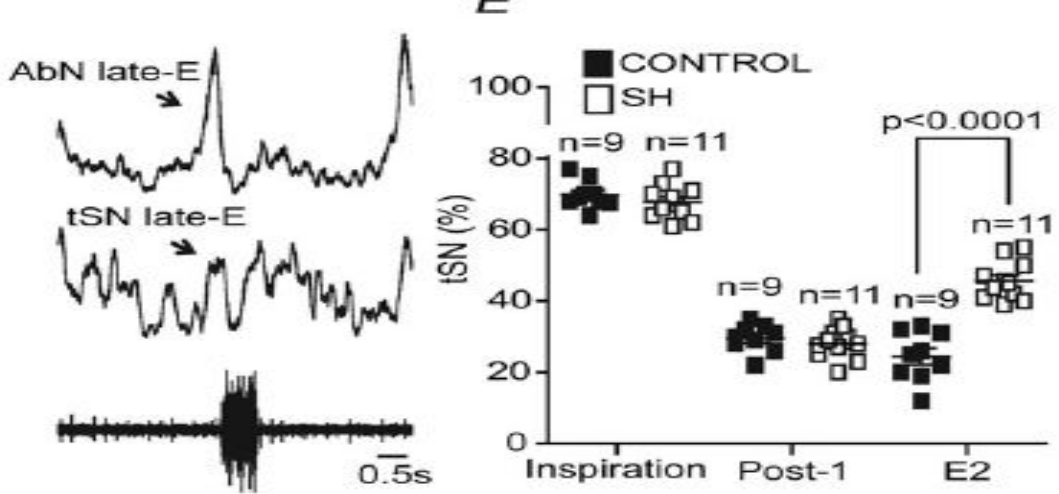

Fig. 8. Respiratory-related modulation of abdominal and thoracic sympathetic neural efferent discharge in rats. A: Integrated $\left(\int\right)$ and raw abdominal nerve discharge (AbN), thoracic sympathetic nerve activity (tSNA), and phrenic nerve discharge (PND) during normocapnia. Abdominal nerve rhythmic bursting exhibits postinspiratory modulation of its rhythmic bursting activity contrasted with late-expiratory activity elicited by subjection to chronic intermittent hypoxia. Thoracic sympathetic nerve bursting manifests late-inspiratory postinspiratory modulated amplification. The phrenic neurogram exhibits regular rhythmic augmentinginspiratory bursting, with only a few diminutive sparse spikes during the postinspiratory epoch (second and third bursts). B: Integrated $\left(\int\right)$ and raw abdominal nerve discharge, thoracic sympathetic nerve activity, and phrenic nerve discharge in rats conditioned with chronic intermittent hypoxia. Subjecting rats to chronic intermittent hypoxia confers late expiratory modulation upon abdominal nerve discharge and coordinately enhances postinspiratory modulation, and elicits phase-spanning late-expiratory-early-inspiratory modulation of thoracic sympathetic nerve discharge. The phrenic neurogram exhibits regular rhythmic augmenting inspiratory bursting, with only a few sparse spikes constituting a diminutive postinspiratory epoch, challenging to discern, but present, in all three bursts. Hypercapnia augments phrenic burst amplitude, contracts duration of the inspiratory epoch, and fails to modulate bursting frequency. C: Expanded views of integrated $\left(\int\right)$ and raw abdominal, thoracic sympathetic, and phrenic nerve bursts during normal conditions. Abdominal nerve bursts evidence post-inspiratory modulation, and thoracic sympathetic nerve bursts evidence phase-spanning late-expiratory post-inspiratory modulation. D: Integrated $\left(\int\right)$ abdominal and thoracic sympathetic and raw phrenic nerve activity in rats conditioned by chronic intermittent hypoxia. Abdominal and thoracic sympathetic nerve activity acquire synchronous late-expiratory modulation. E: Differential fractional distribution of integrated thoracic sympathetic nerve activity across inspiratory, postinspiratory, and late expiratory epochs in normotensive (solid boxes) versus spontaneously hypertensive (open boxes) rats. Thoracic sympathetic nerve discharge exhibits significantly greater amplitude during the late expiratory epoch during chronic intermittent hypoxia compared with normoxic normocapnic conditions. AbdN, abdominal nerve; tSN, thoracic sympathetic nerve; PN, phrenic nerve; SH, spontaneously hypertensive. Modified with permission from Fig. 5 of Moraes et al. (2014a).

and sympathetic oscillations. Mechanical transective lesioning separating the metencephalic and myelencephalic bulks promptly eliminate respirophasic modulation of the sympathetic discharge
(Fig. 12) (Molkov et al., 2014), powerfully substantiating a critical, requisite, and necessary contribution of tonic excitatory synaptic drive deriving from dorsolateral metencephalic units in Kölliker- 
Fuse and medial parabrachial nuclei neurons to Bötzinger complex decrementing postinspiratory neurons (dec post-I) (Dutschmann and Herbert, 2006; Mörschel and Dutschmann, 2009) and from pontine inspiratory expiratory (IE) phase spanning units to rostral ventrolateral medullary presympathetic cells in organizing and mediating sympathorespiratory modulation (Baekey et al., 2010). Accordingly, postinspiratory modulation of rostral ventrolateral medullary presympathetic units may coordinately derive from excitatory synaptic drive conveyed by the correlated spiking of a population of Bötzinger complex glutamatergic decrementing postinspiratory neurons (Sun et al., 1997), heterosynaptic inhibition of inhibitory synaptic drive conveyed to rostral ventrolateral medullary units during the post-inspiratory sub-epoch by Bötzinger complex glycinergic decrementing post-inspiratory units (Sun et al., 1997), and inspiratory-related inhibition of rostral ventrolateral medullary presympathetic units by caudal ventrolateral medullary GABAergic interneurons (Mandel and Schreihofer, 2006) receiving excitatory inputs from higher-order neurons within ventrolateral and interstitial divisions of the nucleus tractus solitarius subnuclei.

\subsection{Caudal ventrolateral medulla}

GABAergic propriobulbar interneurons, interspersed amongst a few glutamatergic propriobulbar interneurons, constitute the caudal ventrolateral medulla, providing the chief and principal inhibitory projection modulating discharge of rostral ventrolateral medullary presympathetic units (Fig. 3) (Ghali, 2017a, 2018; Guyenet, 2006; Mandel and Schreihofer, 2006). Caudal ventrolateral medullary GABAergic interneurons units receive NMDA and non-NMDA glutamatergic excitatory synaptic drive from barosensitive nucleus tractus solitarius units (Mandel and Schreihofer, 2006) and inhibitory synaptic drive from GABAergic propriobulbar interneurons residing within the caudal pressor area (CPA) (Guertzenstein and Silver, 1974). A population of excitatory glutamatergic units within the caudal ventrolateral medulla may convey a glutamatergic drive to rostral ventrolateral medullary presympathetic units (Ghali, 2017a). Caudal ventrolateral medullary GABAergic propriobulbar interneurons exhibiting differential patterns of respiratory-related modifications of unitary activity (Mandel and Schreihofer, 2006) may impose respirophasic modulation upon rostral ventrolateral medullary presympathetic neurons, distributing to the entire sympathetic output (Haselton and Guyenet, 1989; Miyawaki et al., 1995; Molkov et al., 2014). These conjectures are advanced by empirical findings obtained by Baekey et al. (2010) in the in situ brainstem spinal cord preparation of the juvenile rat, the works of Miyawaki et al. (1996) demonstrating abolition of inspiratory-related inhibition of rostral ventrolateral medullary presympathetic neurons by chemical inhibition of the caudal ventrolateral medulla in anesthetized rats (Miyawaki et al., 1996), and computational models generated by Molkov et al. (2014). Botzinger complex glycinergic synaptic inputs may coordinately impose tonic inhibitory modulation upon rostral ventrolateral medullary presymapthetic neurons (see Moraes et al. (2012c) and Sun et al. (1997)), putatively crossmodally augmenting caudal ventrolateral medullary GABAergic inhibition of unit threshold excitability. This hypothesis may be validated by conducting intracellular recordings of RVLM cells and exposing somatodendritic membranes to individual or coordinate microiontophoresis of GABA and/or glycine.

Caudal ventrolateral medullary propriobulbar inhibitory synaptic influences may attenuate the dynamic magnitude of respiropha- sic modulation conferred upon rostral ventrolateral medullary presympathetic units and distributing to sympathetic neural efferent activity (Guyenet et al., 1990; Mandel and Schreihofer, 2006; Miyawaki et al., 1996; Numao et al., 1987). Augmentation of postinspiratory modulation of rostral ventrolateral medullary presympathetic neuronal activity in response to microinjections of pharmacological antagonists of GABA receptors in the rostral ventrolateral medulla and muscimol chemical inhibition of caudal ventrolateral medullary GABAergic interneurons (Mandel and Schreihofer, 2006; Miyawaki et al., 1996; Numao et al., 1987) substantiates the preceding conjecture. Postinspiratory modulation of rostral ventrolateral medullary neurons may be buffered by fast inhibitory synaptic inputs deriving from caudal ventrolateral medullary cells exhibiting analogous postinspiratory modulation of spatiotemporally-variant neuronal spiking dynamics (Mandel and Schreihofer, 2006). Caudal ventrolateral medullary neurons exhibiting postinspiratory modulation may receive the excitatory glutamatergic drive from an excitatory population of Botzinger complex decrementing postinspiratory neurons. They may indirectly impose postinspiratory inhibition upon rostral ventrolateral medullary presympathetic neurons (Mandel and Schreihofer, 2006). The existence of inspiratory- and postinspiratory-related neurons exhibiting neurochemical repertoires alternately consistent with excitatory or inhibitory phenotypes support the presented conjectures (Smith et al., 2007). Further studies are necessary to elucidate an understanding of patterns of neuronal projectivity and crossmodal electrophysiological modulation amongst Bötzinger complex excitatory glutamatergic and inhibitory glycinergic decrementing postinspiratory neurons and rostral ventrolateral medullary propriobulbar and presympathetic bulbospinal neurons and caudal ventrolateral medullary GABAergic interneurons.

\subsection{Nucleus tractus solitarius}

Two bilateral groups of rostrocaudally and mediolaterally organized subnuclei located within the dorsal medulla presenting to the floor of the fourth ventricle constitute the nucleus tractus solitarii (Fig. 2) (Jean, 1992) and prominently contribute to autonomorespirophasic modulation (Zoccal, 2015) in their chief function as a common initial relay for afferent inputs deriving from the periphery (Michelini, 1994; Moreira et al., 2007; Rogers et al., 2000; Takakura et al., 2007). Commissural (Zhang et al., 2011), medial and interstitial (Rogers et al., 2000) and ventrolateral (Von Euler et al., 1973) subdivisions segregate the paired nucleus tractus solitarii into functionally distinct heterotopic zones (Marchenko and Sapru, 2000). Neurons extant within the nucleus tractus solitarii generate autonomorespiratory coupling (Zoccal, 2015) by monomodally or multimodally integrating (Li et al., 1999a,b) oscillatory baroreceptor (Michelini, 1994; Moreira et al., 2007; Rogers et al., 2000; Takakura et al., 2007), chemoreceptor (Zhang et al., 2011), and slow and fast adapting pulmonary stretch receptor (Marchenko and Sapru, 2000) inputs from glossopharyngeal and/or vagal afferents to higher-order neurons in brainstem zones generating the neural breathing pattern, sympathetic oscillations, and cardiovagal premotoneuronal spiking (Molkov et al., 2014). Nucleus tractus solitarius units and efferent neuronal targets thus constitute pathways mediating vasorespiratory and cardiorespiratory crossmodal modulation (Fig. 5) (Molkov et al., 2014; Von Euler et al., 1973). For example, slow (Fenik, 1992; Preobrazhenskiǐ and Fenik, 1989) and fast adapting stretch receptors relay spatiotemporally dynamic alve- 
olar stretch to pump cells (Moreira et al., 2007; Takakura et al., 2007) via vagal afferents encoded in spike cell trains. Nucleus, tractus solitarius pump cells, project to caudal ventrolateral medullary GABAergic interneurons (Mandel and Schreihofer, 2006), which make synaptic contacts with, and convey an inhibitory modulation upon, rostral ventrolateral medullary presympathetic neurons. Conglomerate caudal ventrolateral medullary GABAergic interneuronal spiking, driven by excitatory glutamatergic synaptic drive conveyed from barosensitive nucleus tractus solitarius neurons in response to rises of dynamic arterial pressure magnitude, generate prominent reductions of sympathetic outflow, mediating the sympathetic component of the Hering Breuer reflex (Ghali, 2017a; Guyenet, 2006). Comparatively attenuated respirophasic variation of caudal ventrolateral medullary GABAergic interneuronal basal activity generates congruous respirophasic variations in rostral ventrolateral medullary presympathetic neuronal discharge. Wasserman et al. (2002) demonstrated ventrolateral nucleus tractus solitarius GABAergic interneurons modulate diaphragmatic motor output in urethane- and $\alpha$-chloralose-anesthetized vagotomized spontaneously breathing adult rats. Pulmonary stretch receptor spiking frequency increases in parallel with sympathoinhibitory- mediated reductions of dynamic arterial pressure magnitude and myocardial contraction force and frequency during the inspiratory epoch, synergistically conveying inhibitory modulation upon inspiratoryrelated propriobulbar interneurons and brainstem and spinal motoneurons, generating inspiratory disfacilitation and expiratory augmentation (Breuer, 1868; Daly, 1986; Hering, 1868). Thus, afferents relaying dynamic pulmonary stretch information powerfully modulate breathing, sympathetic oscillations, and cardiovagal premotoneuronal spiking and emergently generate crossmodal coupling amongst the generators.

\section{Respirophasic modulation of cardiosympathetic and cardioparasympathetic output: respiratory sinoatrial dysrhythmia \\ 8.1 Overview}

Neural and hemodynamic variability spectra exhibit high, low, and very-low-frequency oscillations (Guyton et al., 1951; Julien, 2006; Porta et al., 2018; Schweitzer, 1945; Ursino et al., 1992), constituting a mathematically interrogable set of phenomena (Seydnejad and Kitney, 2001) informing mechanisms generating sympathorespiratory modulation amongst and between disparate central oscillators (Ghali and Ghali, 2020). Stephan Hales initially reported oscillatory variability in the crural arterial pressure of a bound mare in 1733 (Hales, 1987). In the 19th century, Traube (1865) and Hering (1869) curiously observed oscillations of dynamic arterial blood pressure magnitude covarying with breathing, having acquired the designation Traube-Hering waves a few years before Hering and Breuer describing and characterizing pulmonary stretch receptor modulation of expiratory and inspiratory phase durations and activities (Breuer, 1868; Hering, 1868). Several years after that, Mayer (1876) observed lower frequency $0.3 \mathrm{~Hz}$ oscillations of dynamic arterial pressure magnitude of anesthetized rabbits, later manifesting in spectra of cardiac interval, arterial resistance, blood flow, and neuronal and neural efferent sympathetic and parasympathetic activity (Julien, 2006). Very low-frequency oscillations previously described to coordinately occur in dynamic arterial blood pressure magnitude and splenic volume (Barcroft and Nisimaru, 1932) were later identified in arteriolar tone (Ursino et al., 1992). Investigating these oscillations has thus proven especially fruitful in uncovering the mechanistic underpinnings of sympathorespiratory, parasympathorespiratory, and sympathoparasympathetic crossmodal modulation (Julien, 2006).

Traube Hering waves constituting the high-frequency band of respiratory-related oscillatory spectral variability evident in neural and hemodynamic variables were seminally described by Traube (1865) and Hering (1869) in the $19^{\text {th }}$ century. Traube-Hering waves expressed in cardiac interval oscillations constitute soi-disant respiratory sinoatrial dysrhythmia (Julien, 2006). We operantly define inspirophasic sinoatrial acceleration to constitute respiratory sinoatrial dysrhythmia. These oscillations may alternately or coordinately be generated by central and/or peripheral mechanisms, several of which are presented with their relative merits and evidence base evaluated (Anrep et al., 1936; Brodie and Russell, 1900; Daly, 1986; Eckberg, 2009; Mendelowitz, 1999). Cardiovagal premotoneuronal disfacilitation likely generates respiratory sinoatrial dysrhythmia (Daly, 1986). Gradual reductions of intrathoracic pressure and increasing negativity of intrapleural pressure occurring dynamically in parallel with the inspiratory epoch generate a gradient augmenting venous return, thus enhancing preload delivered to the atria and stroke volume generated by ventricular contractions (Lansdorp et al., 2014). Increased preload delivered to the ventricles may reflexively reduce myocardial contraction frequency by volume mechanisms or enhance myocardial contraction frequency by activating the Bainbridge reflex (Schroeder and Brehm, 1952; Vatner and Zimpfer, 1981). [N.B.: An increase in afterload presented to the ventricular myocardium induces a reflexive increase in the cardiac contractility, constituting the soi-disant Anrep effect (Anrep and Segall, 1926)]. Importantly, inspirophasic changes in heart rate must be distinguished from changes in heart rate variability occurring during, and paralleling, variations of the respiratory cycle. However, similar and overlapping mechanisms likely coordinately modify both processes (Julien, 2006). The relative balance of sympathetic and parasympathetic inputs to the heart conveyed via cardiac plexus ganglia constitutes the chief neural effector mechanisms determining the rate of the spontaneous depolarization of the soi-disant sinoatrial nodal cell funny current determining discharge frequency and thus heart rate and heart rate variability (Guyenet et al., 2019).

\subsection{Modulation patterns of cardiac vagal preganglionic neurons}

Inspiratory-synchronous disfacilitation of cardiovagal premotoneuronal spiking in the dorsal motor nucleus of the vagus and nucleus ambiguus constitutes the chief mechanism underlying the neurogenesis of respiratory sinoatrial dysrhythmia (Figs. 2 and 3) (Daly, 1986). Accordingly, cardiovagal premotoneurons exhibit nadir spiking frequency during inspiration and a reciprocal peak of spiking frequency during expiration, consistent with the phasepreference of sinoatrial accelerations and decelerations typifying the most common pattern of respiratory sinoatrial dysrhythmia (Daly, 1986). Critically, several studies have paradoxically revealed sinoatrial acceleration occurring during the expiratory epoch in urethaneanesthetized preparations. Though modulatory patterns of cardiovagal premotoneurons residing in the nucleus ambiguus are typically consistent across studies (Neff et al., 2003), exhibiting prominent inspirophasic inhibition and expirophasic facilitation (Gilbey et al., 1984; Mendelowitz, 1999), these units may be preferentially excited 
during inspiratory or expiratory phases.

Inspirophasic peak spiking frequency and post-inspirophasic nadir spiking frequency of cardiovagal premotoneurons (Lindsey et al., 1998) corroborate studies demonstrating inspirophasic sinoatrial deceleration and expirophasic sinoatrial accelerations, though controverts the more commonly observed inspirophasic sinoatrial acceleration (Daly, 1986). Cardiovagal premotoneurons predominantly exhibit expiratory modulation in adult rabbits and cats (Gilbey et al., 1984), inspiratory modulation in urethaneanesthetized adult rats, and inspiratory inhibition in the in vitro preparation of rat medullary brainstem slices (Neff et al., 2003). GABA and glycine receptor modulated signaling mediates elicits inhibitory postsynaptic currents in cardiovagal premotoneuronal somatodendritic neurolemma during the inspiratory epoch(Neff et al., 2003). GABAergic inhibitory modulation of cardiovagal preganglionic neurons may require tonic excitation by $\alpha_{4} \beta_{2}$ nicotinic acetylcholinergic inputs (Neff et al., 2003). Differential patterns of respirophasic modulation of cardiovagal premotoneuronal spiking may result from physiological differences across species, preparation types, and experimental conditions (Gilbey et al., 1984; Jordan et al., 1982; Neff et al., 2003).

\subsection{Mechanisms contributing to modulation of cardiac vagal preganglionic neuronal spiking}

Cardiovagal preganglionic neurons convey acetylcholinergic drive to dendrites and somata of cardiac plexus cardiovagal postganglionic neurons investing the aortic arch and supplying sinoatrial and atrioventricular nodal cells and atrial and ventricular cardiomyocytes (Eckberg, 2003). Fast excitatory glutamatergic and fast inhibitory GABAergic and glycinergic inputs coordinately modulate dynamic membrane voltage trajectories and discharge properties of cardiovagal preganglionic units. Neuroanatomic and neurophysiological evidence variably indicates the putative provision of excitatory drive to cardiovagal premotoneurons deriving from Kölliker-Fuse and medial parabrachial nuclei, Bötzinger complex (Dergacheva et al., 2010), preBötzinger complex (Ellenberger, 1999), and nucleus tractus solitarius units via monosynaptic and/or polysynaptic axonal projections. Several zones may provide inhibitory modulation of cardiovagal premotoneurons Dergacheva et al. (2010); Frank et al. (2009). In a study conducted by (Frank et al., 2009), the photostimulation of transverse brainstem slices containing cardiovagal premotoneurons in nucleus ambiguus identified several zones containing GABAergic interneurons from which inhibitory postsynaptic currents (IPSCs) could be elicited. Regions from which IPSCs could be elicited were located $200 \mu \mathrm{m}$ medial, $400 \mu \mathrm{m}$ medial, $200 \mu \mathrm{m}$ ventral, and $1200 \mu \mathrm{m}$ dorsal and $1000 \mu \mathrm{m}$ medial with respect to patch-clamped ambiguual units (Frank et al., 2009). Retrograde labeling studies may be exploited to determine the neurochemical identity of these zones. Accordingly, pseudorabies virus microinjections in the phrenic nucleus identified units located in the lateral reticular nucleus medial with respect to the nucleus ambiguus cardiovagal premotoneurons (Lois et al., 2009) and nucleus ambiguus fluorogold and rhodamine microinjections labeled magnocellular reticular and lateral paragigantocellular nucleus units (Lois et al., 2009), regions exhibiting overlap with areas from which IPSCs could be elicited and containing units with a predominantly GABAergic neurochemical character (Frank et al., 2009).

Cardiovagal premotoneurons constituting the nucleus ambiguus Figs. 2 and 3 receive a wide convergence of glutamatergic fast ex- citatory and GABAergic fast inhibitory inputs deriving from distributed zones located throughout the brainstem (Dergacheva et al., 2010; Frank et al., 2009). Barosensitive and chemosensitive nucleus tractus solitarius units and pump cells, exhibiting glutamatergic or GABAergic, and/or glycinergic phenotypes project to, and alternately convey excitatory or inhibitory synaptic drive upon, higherorder units and cardiovagal premotoneurons (Moreira et al., 2007; Takakura et al., 2007). Pharmacological GABAergic antagonism of ambiguual cardiovagal premotoneurons abolishes sinoatrial acceleration elicited by rostral ventrolateral medullary stimulation, indicating RVLM may enhance sinoatrial frequency by conveying GABAergic inhibitory modulation upon nucleus ambiguus cardiovagal premotoneurons monosynaptically or through glutamatergic excitation of a pre-ambiguual population of GABAergic units. RVLM-mediated blunting of cardiovagal premotoneuronal spiking, coordinately with the provision of presympathetic descending excitatory inputs to upper thoracic, cardiac preganglionic neurons deriving from the rostral ventrolateral medulla, may constitute a critical mechanism augmenting heart rate. Rostral ventrolateral medullary GABAergic propriobulbar interneurons providing coordinate inputs to Bötzinger complex units (Babic and Ciriello, 2004) and nucleus ambiguus cardiovagal premotoneurons (Babic and Ciriello, 2004) may contribute to crossmodal modulation between and synchrony amongst fast synchronous oscillations generated by these zones. GABAergic propriobulbar interneurons, in turn, may be subjected to excitatory modulation by glutamatergic, cholinergic, and adenosinergic inputs and inhibitory modulation by GABAergic and glycinergic inputs (Neff et al., 2003).

Glycinergic Bötzinger complex decrementing postinspiratory units mediate cardiorespiratory (i.e., parasympathorespiratory) coupling by coordinately conveying inhibitory axodendritic and axosomatic modulation upon cardiovagal premotoneurons (Dergacheva et al., 2010; Frank et al., 2009) and preBötzinger complex preinspiratory, pre-inspiratory-inspiratory phase-spanning, and decrementing early-inspiratory neurons, rostral ventral respiratory group inspiratory neurons, and Bötzinger complex augmenting late expiratory neurons (Molkov et al., 2017; Rybak et al., 2014; Smith et al., 2007). GABAergic Bötzinger complex and augmenting lateexpiratory neurons receive the tonic excitatory drive from glutamatergic retrotrapezoid nucleus units (see Molkov et al. (2017, 2014) and Smith et al. (2009)). Glycinergic Bötzinger complex decrementing post-inspiratory neurons receive the tonic excitatory drive from Kölliker-Fuse and medial parabrachial propriobulbar units residing within the dorsolateral metencephalic tegmentum (Dutschmann and Herbert, 2006; Mörschel and Dutschmann, 2009; Rybak et al., 2014; Smith et al., 2007). Though decrementing Bötzinger complex decrementing post-inspiratory neurons most commonly exhibit a glycinergic neurochemical phenotype, decrementing postinspiratory units may commensurately exhibit an excitatory neurochemical repertoire (Smith et al., 2009). Neurochemical heterogeneity of Bötzinger complex expiratory units indicates a more complex regulation of nucleus ambiguus cardiovagal premotoneurons by respiratory-related units within the core respiratory rhythm generating circuitry (Dergacheva et al., 2010; Frank et al., 2009).

Inspirophasic cardiovagal premotoneuronl IPSCs may be mediated by preBötzinger complex propriobulbar GABAergic and/or glycinergic interneurons (see Morgado-Valle et al. (2010)), constituting a mechanism putatively mediating coupling between pro- 


\section{$5 \% \mathrm{CO}_{2}$ \\ No late-E activity \\ $7 \% \mathrm{CO}_{2}$ \\ $10 \% \mathrm{CO}_{2}$ \\ 1:2 AbN:PN coupling \\ 1:1 AbN:PN coupling}

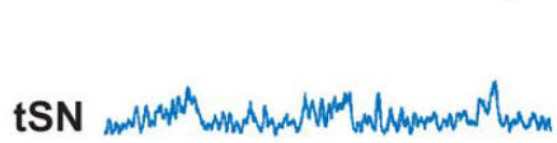
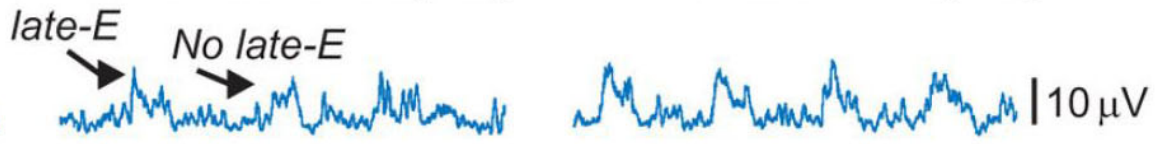

AbN

late-E No late- $E$
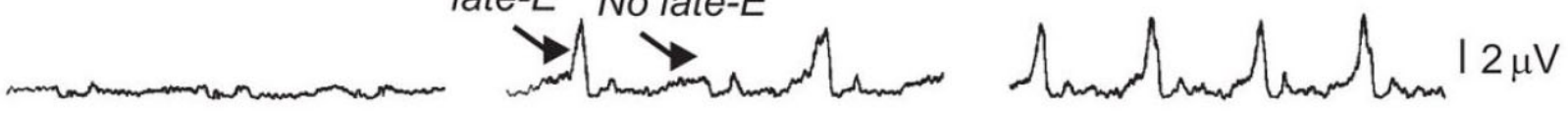

PN
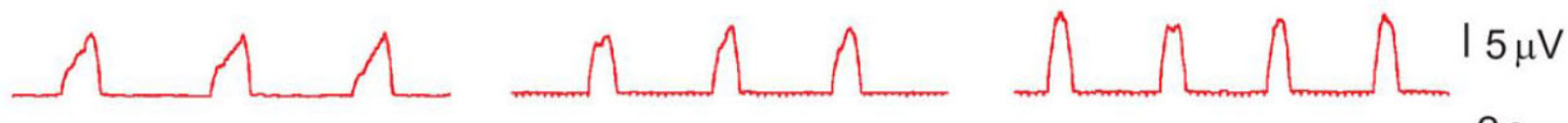

Fig. 9. Thoracic sympathetic, abdominal, and phrenic nerve activity responses to hypercapnia in the in situ arterially-perfused preparation of the unanesthetized decerebrate juvenile rat. Thoracic sympathetic, abdominal, and phrenic nerve integrated activities during normocapnia $\left(5 \% \mathrm{CO}_{2} ;\right.$ left subpanel) and moderate levels of hypercapnia ( $7 \%$ and $10 \% \mathrm{CO}_{2}$; middle and right subpanels). During eucapnic hyperoxia, thoracic sympathetic bursting evidence low amplitude with inspiratory preferential modulation. Abdominal nerve discharge evidences modest inspiratory preferential inhibition. Abdominal and thoracic sympathetic neurograms acquire synchronous late-expiratory bursting with 1: 2 coupling with phrenic nerve bursting in response to $7 \% \mathrm{CO}_{2}$ and synchronous late expiratory bursting with 1: 1 coupling with phrenic nerve bursting in response to $10 \% \mathrm{CO}_{2}$. Top to bottom: thoracic sympathetic nerve (tSN), abdominal nerve $(\mathrm{AbN})$, and phrenic nerve $(\mathrm{PN})$ activities during normocapnia $\left(5 \% \mathrm{CO}_{2}\right)$ and moderate levels of hypercapnia $(7 \%$ and $10 \%$ $\mathrm{CO}_{2}$ ). The $5 \% \mathrm{CO}_{2}$ utilized in the in situ arterially-perfused preparation of the decerebrate juvenile rat represents eucapnia. It recapitulates and replicates the normal metabolic production of carbon dioxide in normothermic animal models in vivo. Thoracic sympathetic and abdominal nerve late-expiratory bursting accelerates quantally the following exposure to progressively augmenting levels of hypercapnia. Abdominal and thoracic sympathetic neurograms occasionally evidence skipping of some late-expiratory bursts at $7 \% \mathrm{CO}_{2}$. Integrated neurogram amplitude expressed in microvolts $(\mu \mathrm{v})$ and timescale bar shown in the right lower hand corner (2 seconds). Modified with permission from Fig. 1 of Molkov et al. (2011).

priobulbar interneuronal microcircuit oscillators and ambiguual cardiovagal premotoneurons. Respirophasic pulmonary stretch may modulate preBötzinger complex propriobulbar GABAergic and/or glycinergic interneuronal spiking via monosynaptic projections or polysynaptic relays through respiratory-related units in the retrotrapezoid nucleus (Guyenet et al., 2019; Takakura et al., 2007) and/or neurons residing within the Kölliker-Fuse or medial parabrachial nuclei (Dutschmann and Herbert, 2006; Mörschel and Dutschmann, 2009; Rybak et al., 2007; Smith et al., 2009). Bötzinger complex decrementing postinspiratory neurons driven by nucleus tractus solitarius pump cells or dorsolateral metencephalic units (Dutschmann and Herbert, 2006; Mörschel and Dutschmann, 2009) may impose inspiratory or postinspiratory preferential modulation upon dynamic cardiovagal premotoneuronal spiking (Daly, 1986). We suggest Bötzinger complex decrementing post-inspiratory units may plausibly coordinately confer respirophasic modulation upon rostral ventrolateral medullary presympathetic units and ambiguual cardiovagal premotoneuronal spiking (Dergacheva et al., 2010). Cardiovagal preganglionic neurons receiving excitatory drive from Bötzinger complex decrementing post-inspiratory neurons and metencephalic elements may generate sinoatrial decelerations during the expiratory phase (Frank et al., 2009; see Dergacheva et al., 2010). Similarly, inspiratory disfacilitation of cardiovagal premotoneurons may contribute prominently to generating sinoatrial accelerations typifying respiratory sinoatrial dysrhythmia (Gilbey et al., 1984; Mendelowitz, 1999).

\section{Strengthening of sympathorespiratory coupling by hypoxia and hypercapnia 9.1 Overview}

Normal breathing during normoxic normocapnia involves active inspiratory chest wall excursions with passive expiratory relaxation of the thoracic cavity (Janczewski et al., 2013). Hypercapnia (Marchenko et al., 2016; Molkov et al., 2011; Zoccal, 2015) and acute and chronic hypoxia (Moraes et al., 2014a, 2012a, 2014b, 2012b,c; Zoccal et al., 2007, 2009a) characteristically elicits active expiratory collapse of the thoracic cavity (Figs. 8-11), empirically evident in abdominal neurogram and electromyogram recordings (Janczewski and Feldman, 2006; Zoccal et al., 2008). The strategy enhances ventilatory carbon dioxide exchange and, in parallel, improves tissue oxygenation (Ghali and Marchenko, 2016b). Experimentally conditioning animals with chronic intermittent hypoxia renders expiratory excursions active during baseline normocapnic normoxia (Moraes et al., 2014b, 2012b; Zoccal et al., 2007, 2009a, 2008). During rest, rostral ventrolateral medullary neurons exhibit varying patterns of respirophasic modulation of neuronal spiking (i.e., inspiratory, postinspiratory, or nonsegmented expiratory), with sympathetic neural efferent activity typically evidencing inspiratory and/or postinspiratory accentuation with late postinspiratory or late expiratory attenuation (Koshiya and Guyenet, 1995; Lipski et al., 1996a,b; Miyawaki et al., 1996; Zoccal et al., 2008). Hypercapnia (Molkov et al., 2014) and acute and chronic hypoxia (Moraes et al., 2014b, 2012b; Zoccal et al., 2007, 2009a, 2008) powerfully enhances the strength of respirophasic modulation and sympathetic neural burst amplitude and elicit synchronous late-expiratory sym- 
pathetic and abdominal nerve bursting. Distinct mechanisms are mediating evident respiratory neuroplasticity in in vivo and situ preparations of the anesthetized, unanesthetized decerebrate, and conscious (Moraes et al., 2013) adult and juvenile (Zoccal et al., 2007, 2009a, 2008) rats. In general, rats subjected to either hypercapnic (Molkov et al., 2014) or acute or chronic normocapnic hypoxia develop sustained amplifications of dynamic arterial pressure magnitude, correlated with parallel increases in sympathetic neural efferent activity and plasma levels of catecholamines (Zoccal et al., 2007, 2009a, 2008), with the genesis of contemporaneous and synchronous late-expiratory bursting manifest in sympathetic and expiratory abdominal nerve bursting (Molkov et al., 2014). We dissect mechanisms mediating, and contributing to, hypercapniaand hypoxia-induced amplification of the strength of respirophasic variation of discharge properties of the brainstem and spinal cord sympathetic-related units and modulation of sympathetic neural efferent activity (Molkov et al., 2017; Zoccal, 2015).

\subsection{Hypercapnia}

Hypercapnia elicits late-expiratory burst-clustered spiking in abdominal nerve correlated, coherent, and synchronous with lateexpiratory modulation of sympathetic neural efferent activity (Figs. 8-11) (Molkov et al., 2014), consistently demonstrated across in vivo and situ preparations. The behavior increases quantally, paralleling increasing levels of hypercapnia, eventually becoming coupled 1: 1 with phrenic nerve discharge (Abdala et al., 2009; Molkov et al., 2010). In the in situ brainstem spinal cord preparation of the unanesthetized decerebrate juvenile rat, hypercapnia elicits quantal amplification of synchronous late-expiratory bursting, coordinately manifest in sympathetic and abdominal neural efferent activity (Molkov et al., 2014). Amplification of late-expiratory quantally synchronized across abdominal nerve and thoracic sympathetic bursting paralleled increasing levels of arterial blood carbon dioxide. At 5\% hypercapnia, abdominal-sympathetic-synchronized lateexpiratory bursting exhibited 1: 2 at 5\% hypercapnia and 1: 1 coupling with phrenic nerve discharge at $7 \%$ and $10 \%$ hypercapnia see Molkov et al. (2011).

Pharmacological inactivation of the retrotrapezoid nucleus and parafacial respiratory group zone abolishes hypercapnia-induced late expiratory abdominal nerve bursting (Abdala et al., 2009); inhibition of Phox $2 b$ transcription factor expressing neurons elicits a similar effect (Marina et al., 2010). Retrotrapezoid nucleus Phox $2 b$ transcription factor-expressing glutamatergic chemosensitive units (Guyenet et al., 2019) convey excitatory axodendritic and axosomatic synaptic drive upon bulbospinal expiratory cells residing within the caudal ventral respiratory group, driving late expiratory bursting in abdominal nerve discharge (Abdala et al., 2009; Molkov et al., 2010). These findings indicate late-expiratory bursting coherent amongst abdominal and sympathetic neural efferent discharge induced by hypercapnia (Molkov et al., 2014) originates from a common source, putatively from chemosensitive retrotrapezoid nucleus units and spontaneously bursting parafacial respiratory group preinspiratory units (Abdala et al., 2009; Molkov et al., 2010), with cells in these zones exhibiting isoelectric neural silence at $5 \% \mathrm{CO}_{2}$ (considered normocapnia in hypothermic in situ preparations) and acquiring remarkable bursting only when exposed to higher levels of carbon dioxide tension (Abdala et al., 2009; Molkov et al., 2010). Interactions amongst overlapping Botzinger complex and rostral ventrolateral medullary propriobulbar interneuronal mi- crocircuit oscillators (Moraes et al., 2012c; Sun et al., 1997) may also generate hypercapnia and/or hypoxia-mediated accentuation of sympathorespiratory coupling. Experimental collectively indicates chemosensitive retrotrapezoid nucleus cells (Guyenet et al., 2019; Molkov et al., 2017) and parafacial respiratory group pre-inspiratory microcircuit oscillators (Onimaru et al., 2018) strengthen hypercapnia and/or acute and chronic hypoxia-mediated sympathorespiratory coupling (Abdala et al., 2009; Molkov et al., 2010), by providing coordinate inputs to caudal ventral respiratory group expiratory premotoneuronal bulbospinal units and rostral ventrolateral medullary presympathetic units (Molkov et al., 2014). Molkov et al. (2011) describe a model detailing mechanism putatively underlying sympathorespiratory crossmodal modulation building upon the work dos Baekey et al. (2010) and the group's previously developed models see Molkov et al. (2010). Molkov's models incorporate a population of RTN and pFRG conveying common tonic excitatory drive to respiratory and sympathetic generators (Molkov et al., 2017). Accordingly, Bötzinger complex expiratory neurons impose various patterns of respirophasic modulation upon rostral ventrolateral medullary unitary spiking frequency according to the state of the network (i.e., whether exposed to normocapnic normoxia, hypercapnia, or hypoxia) and predecessor exposures to chronic intermittent hypoxic conditioning, a mechanism which powerfully and emergently generates sympathorespiratory crossmodal coupling (Moraes et al., 2012a,b,c; Zoccal et al., 2008) Bötzinger complex and rostral ventrolateral medullary neurons are intermingled, exhibit significant overlap, and make extensive heterologous neurosynaptic contacts (see Moraes et al. (2012c) and Sun et al. (1997)). Bötzinger complex decrementing post-inspiratory or augmenting late-expiratory neurons making synaptic contacts with rostral ventrolateral medullary presynaptic membranes could influence intermediolateral cell column preganglionic sympathetic neuronal spiking frequency.

To further resolve relative contributions of Phox $2 b$-expressing chemosensitive glutamatergic retrotrapezoid nucleus cells and parafacial respiratory group pre-inspiratory neurons to synaptic neuroplasticity of sympathetic, expiratory, and inspiratory oscillators, Zoccal et al. (2018). determined the effects of $10 \%$ hypercapnia upon thoracic sympathetic, abdominal. Phrenic nerve bursting before and following selective ablation of neurokinin 1-expressing retrotrapezoid neurons utilizing substance $\mathrm{P}$-saporin conjugate and pharmacological antagonism of parafacial respiratory group neuronal glutamate receptors utilizing kynurenic acid. Lesioning neurokinin 1-expressing glutamatergic retrotrapezoid nucleus units by microinjecting substance P saporin-conjugate significantly attenuated the magnitude of late-expiratory bursting synchronized across thoracic sympathetic and abdominal neurograms and amplification of phrenic inspiratory bursting elicited by $10 \%$ hypercapnia in the in situ arterially perfused preparation of the unanesthetized decerebrate juvenile rat (Zoccal et al., 2018). In contrast, pharmacological antagonism of parafacial respiratory group glutamate receptor modulated signaling to utilize microinjections of the nonselective glutamate receptor antagonist kynurenic acid $(100 \mathrm{mM})$ completely abolished late-expiratory bursting synchronized across thoracic sympathetic and abdominal nerve discharge, though failed to modify phrenic nerve responses to hypercapnia in this preparation (Zoccal et al., 2018). The results indicate an absolute requirement of parafacial respiratory group units constituting the expiratory oscillator and the critical importance of chemosensitive glutamatergic retrotrape- 
zoid nucleus neurons in generating synchronous late-expiratory bursting in thoracic sympathetic and abdominal nerve in response to chemoreceptor stimulation (Zoccal et al., 2018). Accordingly, the results appear to be most consistent with a model whereby hypoxia and hypercapnia-induced amplification of phrenic inspiratory amplitude and frequency critically require drive-by chemosensitive retrotrapezoid nucleus units, though remains essentially independent of parafacial respiratory group unitary activity.

\subsection{Hypoxia}

Acute and chronic intermittent hypoxia potently elicit lateexpiratory bursting synchronized across abdominal and thoracic sympathetic neural efferent discharge (Figs. 8-11) (Moraes et al., 2014a,b, 2012b; Zoccal et al., 2007, 2009a, 2008). Acute hypoxia specifically augments thoracic splanchnic sympathetic nerve activity during the early or late segments of the expiratory epoch (see Moraes et al. (2014a)). Chronic intermittent hypoxic conditioning elicits a pattern of long term facilitation in propriobulbar interneuron microcircuit oscillators generating the breathing pattern and sympathetic activity typified by late-expiratory bursting synchronized across abdominal and thoracic sympathetic neurograms (Moraes et al., 2014b). Late-expiratory sympathetic bursting may amplify the sympathetic vasoconstrictor tone conveyed to the vessels during active expiratory efforts (Gilbey, 2007). Chronic intermittent hypoxic conditioning elicits long-term facilitation in phrenic motor output by amplifying the resting amplitude of population phrenic motoneuronal discharge (Fuller et al., 2003), chiefly utilizing serotonergic neuromodulation. An intimate understanding of these mechanisms may prove useful in the rational therapeutic design of novel strategies effectively ameliorating respiratory dysfunction in the setting of injury to the bulb and spinal cord (Fuller et al., 2003; Ghali, 2017c). Chronic intermittent hypoxia experimentally imposed or pathologically experienced by individuals suffering from central and/or obstructive sleep apnea elicits arterial hypertension, likely resulting from originating long-term potentiation of the glutamatergic retrotrapezoid nucleus and commissural nucleus tractus solitarius units conveyed to the medullary lateral tegmental field and rostral ventrolateral medullary propriospinal interneuronal microcircuit oscillators. Individuals developing arterial hypertension in the setting of chronic intermittent hypoxia may exhibit increased dynamic gain and sensitivity of the peripheral chemoreflex and baroreflex and augmented basal and reflexogenic sympathoexcitation and neurogenic arterial hypertension (Molkov et al., 2011; Zoccal and Machado, 2011).

Afferent denervation of the chemosensitive carotid bodies eliminates neither the development of late-expiratory bursting activity synchronized across abdominal and sympathetic neural efferent discharge nor sympathetic potentiation in in situ arteriallyperfused unanesthetized decerebrate juvenile rat previously conditioned with chronic intermittent hypoxia (Zoccal et al., 2008). Reducing chemosensory drive coordinately and significantly reduces late-expiratory synchronous bursting in abdominal and thoracic sympathetic neural discharge, effects evaluated in the in situ preparation of the arterially-perfused unanesthetized decerebrate juvenile rat, inspiring the wise construct of computational models by Molkov et al. $(2014,2011)$ capturing this behavior accordingly (Molkov et al., 2011). The supraspinal genesis of late-expiratory synchronous bursting in abdominal and sympathetic neural efferent discharge elicited by hypoxia represents coupled events (Molkov et al., 2014). Mechanisms strengthening sympathorespiratory coupling in response to oxygen deprivation may include altered sensitivity of peripheral chemoreceptor glomus cells, membrane voltage trajectories of which become depolarized by moderate and severe levels of hypoxia (Lahiri et al., 2006). Hypoxia generates an electrophysiological spike train in glossopharyngeal and vagal afferents relayed centrally, filtered, and integrated by, nucleus tractus solitarius neurons (Machado, 2001). Computational processing of these dynamic oscillatory inputs modulates the neural breathing rhythm, sympathetic oscillations, and ambiguual cardiovagal premotoneuronal spiking (Moraes et al., 2014b, 2012b; Zoccal et al., 2007, 2009a, 2008). Hypoxia could strengthen sympathorespiratory coupling (Moraes et al., 2014b, 2012b; Zoccal et al., 2007, 2009a, 2008) by directly activating or sensitizing central and/or peripheral chemoreceptors (Blain et al., 2010). Hypoxia generates hydrogen ion oxidizing equivalents in the microenvironmental milieu of chemosensitive glutamatergic retrotrapezoid nucleus units by preferentially favoring steps of the glycolytic pathway preceding prefacing soi-disant oxidative phosphorylation. Compensatory amplification of lactate dehydrogenase mediated conversion of pyruvate to lactate to regenerate $\mathrm{NAD}+$ oxidizing equivalent to sustain adenosine triphosphate generation, which may enhance glutamatergic RTN unit discharge frequency, coordinately enhancing Botzinger complex and rostral ventrolateral medullary neuronal spiking (Molkov et al., 2014). A similar set of studies conducted in the unanesthetized decerebrate preparation of the rat will elucidate the mechanisms underlying hypercapnia, acute hypoxia, and chronic intermittent hypoxia-mediated strengthening of sympathorespiratory coupling (see Ghali and Marchenko (2016b) and Marchenko et al. (2016) for precedence).

In the presence of pharmacological inhibition of the retrotrapezoid nucleus and parafacial respiratory group, pharmacological activation of peripheral chemoreceptors fails to elicit late-expiratory bursting synchronized across abdominal and sympathetic neural efferent discharge in the in situ brainstem spinal cord preparation of the unanesthetized decerebrate juvenile rat (Moraes et al., 2012a). These results imply chronic intermittent hypoxia-mediated genesis of late-expiratory synchronous bursting in abdominal and sympathetic neurograms could be mediated by coordinate synaptic inputs conveyed by chemosensitive glutamatergic retrotrapezoid nucleus units and parafacial respiratory group pre-inspiratory oscillators or neurosynaptic coupling of Bötzinger complex decrementing post-inspiratory and augmenting late-expiratory neurons and rostral ventrolateral medullary propriobulbar interneuronal microcircuit oscillators and presympathetic bulbospinal units (Molkov et al., 2014; Zoccal et al., 2008). Enhanced Bötzinger complex and rostral ventrolateral medullary neuronal spiking may express the composite influences of enhanced synaptic drive conveyed from the retrotrapezoid nucleus and parafacial respiratory group, sensitization of metencephalic and myelencephalic chemoreceptors by peripheral chemoreceptor inputs (Guyenet et al., 2019), or blunted inhibitory drive from Bötzinger complex postinspiratory neurons (Molkov et al., 2010), compromised by hypoxic step reduction of the subpopulation of functionally active GABA and glycine receptor modulated neurosynaptic interactions (see Ghali and Ghali (2020)). These neuroplasticity mechanisms may utilize serotonergic (Mulkey et al., 2007) or purinergic (Mulkey et al., 2006) neuron transduction or be generated by oxidative stress. Accordingly, Barnett et al. (2017) provide a conceptual and computational model expressing interac- 


\section{$5 \% \mathrm{CO}_{2}$ \\ 1:2 AbN:PN coupling No late-E \\ $7 \% \mathrm{CO}_{2}$ \\ 1:1 AbN:PN coupling}

tSN

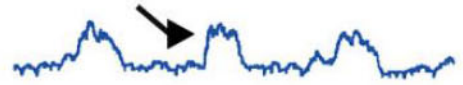

$10 \% \mathrm{CO}_{2}$
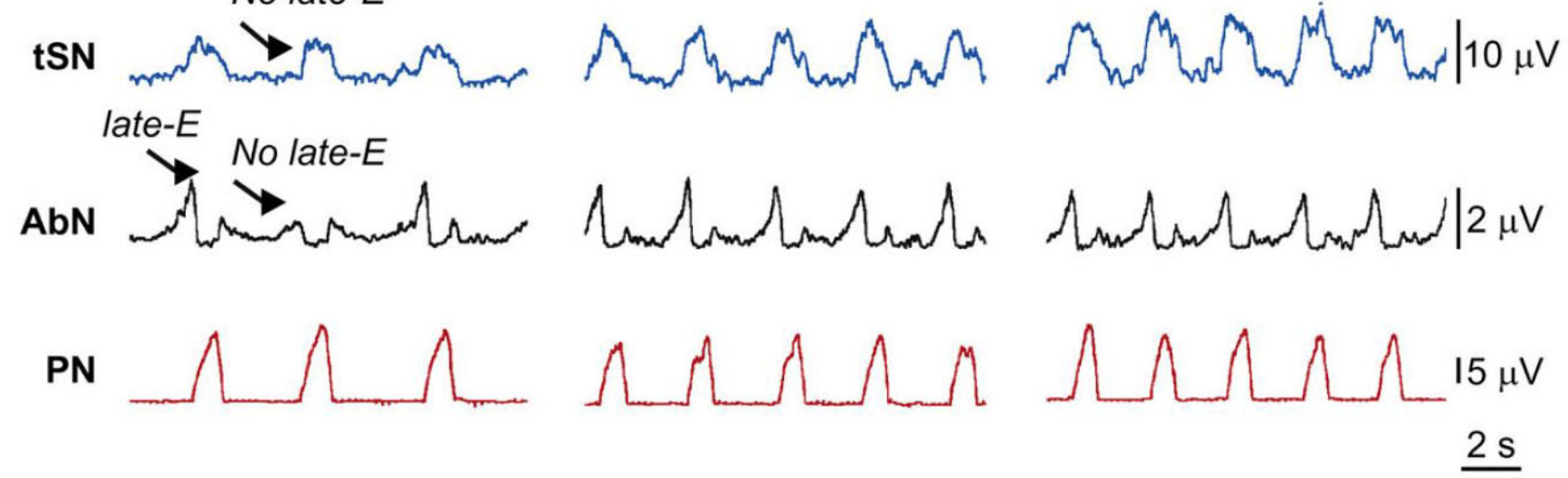

Fig. 10. Thoracic sympathetic, abdominal, and phrenic nerve activity responses to hypercapnia in in situ arterially-perfused preparation of the unanesthetized decerebrate juvenile rats having undergone chronic intermittent hypoxic conditioning. Thoracic sympathetic, abdominal, and phrenic nerve activities during normocapnia ( $5 \% \mathrm{CO}_{2}$; left subpanel) and variable levels of hypercapnia ( $7 \%$ and $10 \% \mathrm{CO}_{2}$; middle and right subpanels) are demonstrated. Abdominal and thoracic sympathetic neural efferent activity exhibit late-expiratory synchronous bursting with 1: 2 coupling into the presence of $5 \%$ $\mathrm{CO}_{2}$ (left subpanel) and acquire amplified synchronous late-expiratory bursting with 1:1 coupling in response to modifying carbon dioxide tension within the perfusate to $7 \%$ and $10 \% \mathrm{CO}_{2}$ (middle and right subpanels) Top to bottom: thoracic sympathetic nerve (tSN), abdominal nerve (AbN), and phrenic nerve (PN) activities during normocapnia $\left(5 \% \mathrm{CO}_{2}\right)$ and hypercapnia $\left(7 \%\right.$ and $\left.10 \% \mathrm{CO}_{2}\right)$. Use of $5 \% \mathrm{CO}_{2}$ ontogenically recapitulates and substitutes interstitial carbon dioxide concentrations generated by biochemical metabolism by normothermic animals in vivo under the hypothermic state employed in arterially-perfused animals in situ. Integrated neurogram amplitude expressed in microvolts $(\mu \mathrm{v})$ and timescale bar shown in the right lower hand corner ( 2 seconds). Modified with permission from Fig. 1 of Molkov et al. (2011).

tions amongst and between elements of the respiratory, central pattern generator with central and peripheral chemoreceptor mechanisms, reasonably conjecturing and presupposing amplification of sympathorespiratory coupling and the development of neurogenic arterial hypertension and active expiration at rest elicited by chronic intermittent hypoxia may result from neuroplasticity mechanisms predicated upon neuronal sensitization and upregulation of somatodendritic membrane ion channel conductance.

\subsection{Chemoreceptor vagal modulation}

Dynamic spiking of vagal axons transducing alveolar stretch tempers hypercapnia mediated augmentation of phrenic burst amplitude in unanesthetized decerebrate (Ghali and Marchenko, 2016b) and urethane-anesthetized (Lemes and Zoccal, 2014; Takakura et al., 2007) rats and peripheral chemosensory facilitation of sympathetic neural bursting (vis-à-vis Ghali and Marchenko, 2016b). Dynamic vagal axonal spiking may similarly powerfully modulate the strength of sympathorespiratory coupling elicited by hypercapnia and hypoxia (Ghali and Marchenko, 2016b). [N.B.: The strategy of soi-disant "permissible hypercapnia" in mechanicallyventilated patients in the intensive care unit being managed according to ARDS protocol constitutes a natural pressor synergistically enhancing intravenously administered phenylephrine, norepinephrine, dopamine, and/or vasopressin, though paradoxically hindering the inotropic effects of these agents by generating a mild systemic acidosis (inotropic agents elicit cardiac contractility with less potency in the presence of tissue acidosis), coordinately supporting blood pressure and rendering attempts at weaning from mechanical ventilation more likely to be successful]. Crossmodal modulation amongst central and peripheral chemoreceptor and nucleus tractus solitarius pump cells, evident across a variety of ani- mal models, preparation types, and experimental conditions (Ghali and Marchenko, 2016b; Lemes and Zoccal, 2014; Takakura et al., 2007), may contribute to emergently generating sympathorespiratory coupling (see excellent and concise review by Zoccal (2015)). In urethane-anesthetized rats, vagotomy powerfully enhances the amplitude of expiratory abdominal nerve bursting elicited by the administration of hypercapnic and hypoxic gas mixtures (Lemes and Zoccal, 2014). Dynamic and tonic vagal influences powerfully differentially modulate hypercapnia-induced augmentation of hypoglossal pre-inspiratory and hypoglossal and phrenic inspiratory bursting amplitude in unanesthetized decerebrate adult rats (Ghali and Marchenko, 2016b), effects powerfully attenuated by vagotomy (Ghali and Marchenko, 2016b). GABAergic nucleus tractus solitarius pump cells inhibitory axodendritic and axosomatic modulation of chemosensitive glutamatergic retrotrapezoid nucleus units may contribute to pulmonary stretch-mediated attenuation of supraspinal chemoreceptor neuronal spiking elicited by hypercapnia and/or hypoxia in the presence of vagal continuity (Takakura et al., 2007). Constitutively spiking vagal afferents may restrict the preinspiratory, and attenuate the inspiratory, component of hypoglossal inspiratory bursting (Ghali, 2015; Ghali and Marchenko, 2016b).

\section{Respiratory gating of baromodulation of sympathetic oscillations}

Sympathetic oscillators receive the powerful modulatory synaptic drive from barosensitive nucleus tractus solitarius units (Fig. 5) (Mendelowitz, 1999). This constitutes a negative feedback mechanism regulating dynamic arterial pressure magnitude, dumping of spurts of blood into the microcirculation, and tissue perfusion dynamics (Alexander and Cuir, 1963). Dynamic arterial pressure rises 
Control

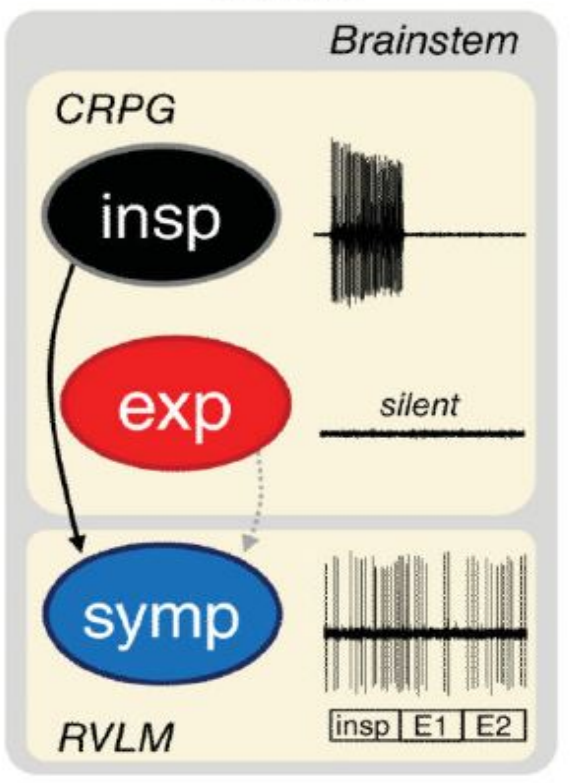

\section{$\mathrm{ClH}$}

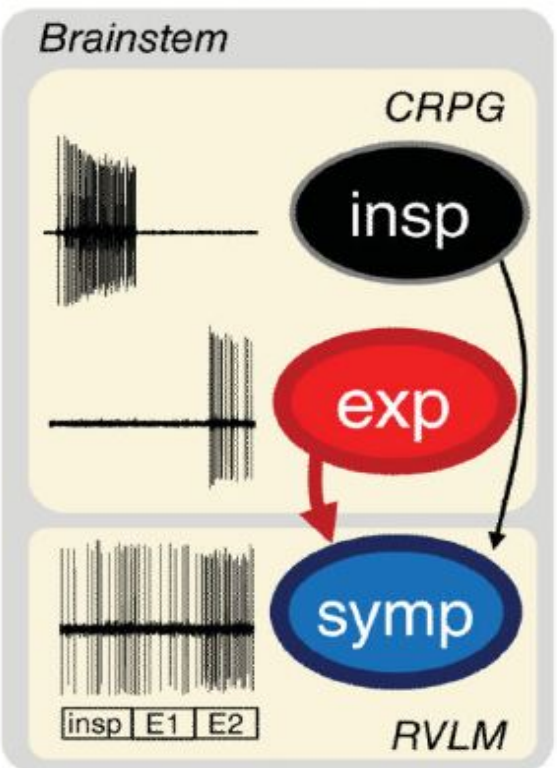

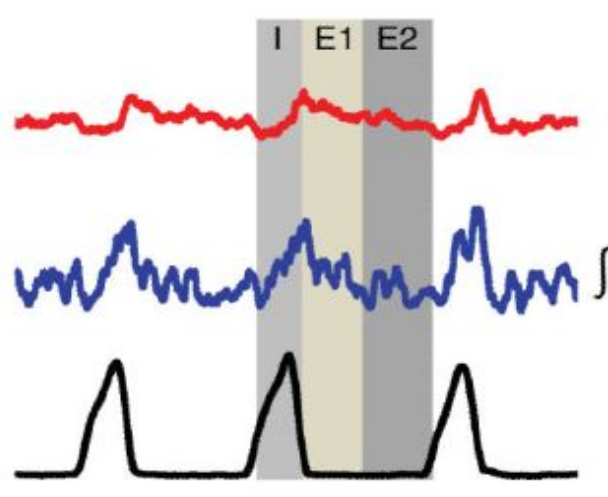

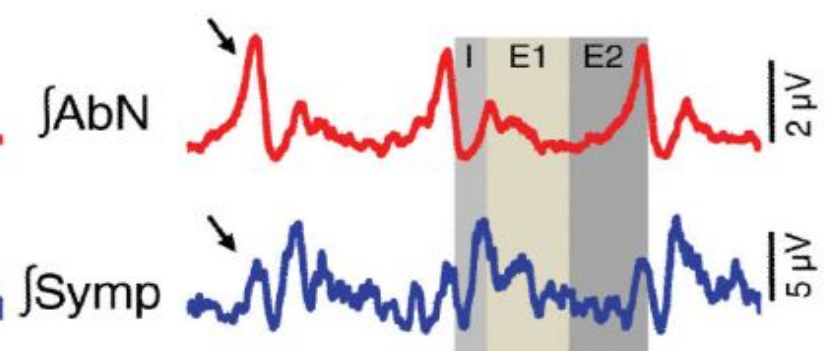

SPhr

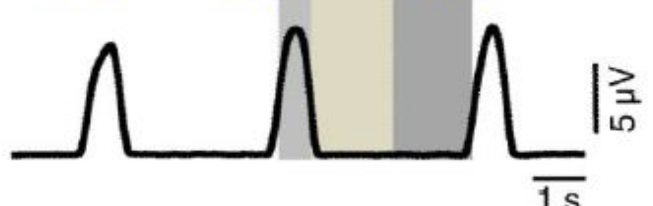

Fig. 11. Network changes contributing to modifications of sympathorespiratory coupling following chronic intermittent hypoxic (CIH) conditioning. Traces within the boxes in the upper aspect of the left and right panels represent inspiratory-related, expiratory-related, and rostral ventrolateral medullary neuronal extracellular recordings. Integrated $\left(\int\right.$ ) recordings of abdominal $(\mathrm{AbN})$, sympathetic (Symp), and phrenic (Phr) nerve discharge are depicted by the lower traces. Sympathetic neural efferent activity exhibits phasic increases during the inspiratory phase (insp), with peak spiking frequency occurring during the late inspiratory and early post-inspiratory epochs (post-I), followed by dynamic reductions of bursting amplitude during the late post-inspiratory and late-expiratory epochs (E2) in the presence of normoxic normocapnia (left panels). Inputs deriving from inspiratory- (insp) and expiratory- (exp) related neurons constituting the soi-disant central respiratory pattern generator (CRPG) to rostral ventrolateral medullary (RVLM; (Moraes et al., 2012b) presympathetic neurons (symp) form and contribute to the emergent generation of the respiratory pattern. Inspiratory chest excursions constitute a dynamic, active process during normoxic normocapnia, with expiratory relaxation of the thoracic cavity occurring passively. In rats subjected to chronic intermittent hypoxia, propriobulbar interneuronal microcircuit oscillators generating active expiration become constitutive during resting normoxic normocapnia (Molkov et al., 2011; Zoccal et al., 2008). Consequently, expiratory neurons, which natively exhibit isoelectric neural silence during rest, become active and supply excitatory neuronal inputs to bulbospinal expiratory units and rostral ventrolateral medullary presympathetic neurons, leading to the emergence and development of coupled and synchronous late-expiratory bursting activity in the discharge of abdominal and sympathetic nerve outputs (indicated by the arrows). Modified with permission from Fig. 1 of Zoccal (2015).

to elicit sinoatrial deceleration and corrective attenuation of arteriolar and venular tone via enhanced oscillatory baroreceptor spiking frequency, coordinated by reductions in the sympathetic drive to the heart and vessels and increases of ambiguual cardiovagal premotoneuronal drive to electroconductive circuitry of the heart and atrial myocardium (Rogers et al., 2000). Baroreceptors sensitive to dynamic arterial stretch are most densely located within the aortic arch and carotid sinus, though may be found sparsely distributed throughout middle internal carotid (Toorop et al., 2013), vertebral (Agadzhanian and Kupriianov, 2008; Kupriyanov, 2009), and subclavian arteries (Chevalier-Cholat and Friggi, 1977, 1976; Kidd et al., 1974). Baroreceptor and baroafferent discharge frequency covary with dynamic arterial pressure magnitude. Oscillatory baroreceptor inputs centrally-conveyed to medial and interstitial nucleus 
tractus solitarius units via the aortic depressor nerve, carotid sinus nerve, and distributed afferent fibers join cranial nerves en route to myelencephalic oscillators may coordinately receive inputs from Hering's nerve and vagal afferents relaying fast oscillatory chemoreceptor inputs and vagal afferents relaying slow oscillatory alveolar stretch. Multimodal barosensitive, chemosensitive, and/or pulmonary stretch mechanosensitive units residing within the nucleus tractus solitarius may constitute a mechanism of respiratory gating and multiplicatively synergistic chemoreceptor augmentation of oscillatory baroreceptor inputs to higher-order neural networks. Nucleus tractus solitarius propriobulbar interneuron microcircuit oscillatory convey synaptic drive to caudal ventrolateral medullary GABAergic units mediating state-dependent respirophasic and "baro-phasic" inhibition of rostral ventrolateral medullary presymapthetic neurons, eliciting contemporaneous and parallel amplification of ambiguual cardiovagal premotoneuronal spiking (Ghali, 2017a, 2018; Guyenet, 2006). The magnitude of sinoatrial deceleration in response to a given incremental augmentation of dynamic arterial pressure magnitude constitutes the chief metric characterizing baroreflex gain (Rogers et al., 2000), powerfully crossmodally modulated by neural respiratory rhythmicity and dynamic changes in pulmonary stretch (Baekey et al., 2010), along with spectral peaks nestled within dynamic arterial pressure magnitude and cardiac interval. The magnitude of sinoatrial deceleration elicited by a fixed rise of dynamic arterial pressure varies with respect to respiratory cycle phase (Baekey et al., 2010), implying phase-dependent differential stimulability or inhibitability of sympathetic and cardiovagal preganglionic premotoneuronal spiking, with peak unitary discharge frequency occurring during the period which they are most excitable (Rogers et al., 2000). Sinoatrial decelerations elicited by the baroreflex are preferentially more prominent during the early expiratory epoch when afferents are most activated (Gilbey et al., 1984).

Expiratory-related neurons exhibiting arterial pulse modulation indicates baromodulation of the expiratory epoch and supports barostimulation facilitates expiratory-related neural activity. Lindsey et al. (1998) demonstrated the inspiratory phase contracts in response to chronically elevated dynamic arterial pressure magnitude. Metencephalic zones gate baroreceptor influences upon the propriobulbar interneuronal microcircuit oscillators generating breathing. Accordingly, barosensitive nucleus tractus solitarius units receive modulatory synaptic drive preferentially exhibiting higher spiking frequency during the inspiratory epoch from neurons constituting dorsolateral metencephalic nuclei (Molkov et al., 2014). Electrical stimulation of propriobulbar interneuronal microcircuit oscillators constituting the Kölliker-Fuse nucleus reduces the barosensitivity of monomodally or multimodally sensitive nucleus tractus solitarius cells in response to carotid sinus oscillatory inputs. Enhanced metencephalic neuronal spiking frequency specifically reduces baroreceptor gain during the inspiratory epoch (Brunner et al., 1982; Dove and Katona, 1985; Li et al., 1999b,a; Lindsey et al., 1998). These findings illustrate propriobulbar interneuronal microcircuit oscillator inputs to sympathetic oscillators, and ambiguual cardiovagal premotoneurons constitute critical mechanisms mediating sympathorespiratory modulation (Molkov et al., 2017).

\section{Baromodulation of neural breathing}

Respiratory-related unitary spiking subjected to baromodulation constitutes a mechanism emergently generating sympathorespira- tory coupling (see Lindsey et al. (1998)). Extracellular recordings of brainstem respiratory-related units exhibit pulse-synchronous modulation in vagotomized unanesthetized decerebrate cats (Lindsey et al., 1998). Higher unitary discharge frequency during the expiratory epoch parallels augmentations of dynamic arterial pressure magnitude (Lindsey et al., 1998). Oscillatory baroreceptor inputs modulate respiratory-related neuronal spiking occurring in response to dynamic changes of arterial pressure magnitude, coordinately with evidence provided validating supraspinal mechanisms generate the described coupling (Morrison et al., 1984). Accordingly, in the unanesthetized decerebrate juvenile cat, vagotomy significantly reduces the pulse-synchronous modulation of pontine respiratory-related neurons by the arterial pressure waveform, contemporaneously amplifying the augmenting inspiratory pattern of respirophasic modulation of the units (Dick et al., 2009). Barostimulation elicits barosensitive medial and interstitial glutamatergic nucleus tractus solitarius neuronal spiking, which reduces sympathetic activity by activating a population of GABAergic interneurons residing within the caudal ventrolateral medulla conveying inhibitory axodendritic and axosomatic drive to rostral ventrolateral medullary propriobulbar interneuronal microcircuit oscillators and interneuronally-coupled presympathetic bulbospinal cells (Dampney, 1994; Ghali, 2017a, 2018; Goodchild and Moon, 2009; Guyenet et al., 1990). As a parallel pathway, barosensitive glutamatergic nucleus tractus solitarius units convey excitatory synaptic drive to Bötzinger complex glycinergic decrementing postinspiratory neurons, mediating coordinate enhancement of rostral ventrolateral medullary presympathetic and Bötzinger complex expiratory neuronal spiking, imposing baromodulation upon the respiratory rhythm and mediating sympatho-expiratory coupling (Baekey et al., 2010; Molkov et al., 2014).

Barostimuli elicits differential effects upon neural breathing pattern, whether applied during the inspiratory versus expiratory epochs, effects prominently demonstrated in the in situ arteriallyperfused preparation of the unanesthetized decerebrate juvenile rat (Figs. 13 and 14) (Baekey et al., 2010). The behavior elucidates an understanding of interactions amongst oscillatory baroreceptor inputs with propriobulbar interneuronal microcircuit oscillators generating breathing and indicate the latter gate the former. Barostimuli delivered during neural inspiration often fail to modify phrenic burst pattern, though successfully eliminate respirophasic modulation of sympathetic neural activity (Baekey et al., 2008). In contrast, barostimuli delivered during neural postinspiration and late expiration augment expiratory neural activity and eliminate respirophasic modulation of sympathetic neural efferent discharge (Baekey et al., 2008), supporting a model predicated upon prominent inhibition conveyed to Bötzinger complex decrementing postinspiratory neurons by decrementing early inspiratory neurons residing within the and rostral ventral respiratory group during neural inspiration (Molkov et al., 2017; Smith et al., 2007), preventing barostimuli delivered during inspiration from eliciting modifications of phrenic nerve bursting.

Baekey et al. (2010) present a characterizing model mechanism underlying the baromodulation of propriobulbar interneuronal microcircuit oscillators generating neural breathing. According to this model, predicated upon reciprocal interaction of decrementing postinspiratory neurons with augmenting expiratory neurons, two classes of barosensitive nucleus tractus solitarius neurons included one group conveying synaptic drive to caudal ventrolat- 

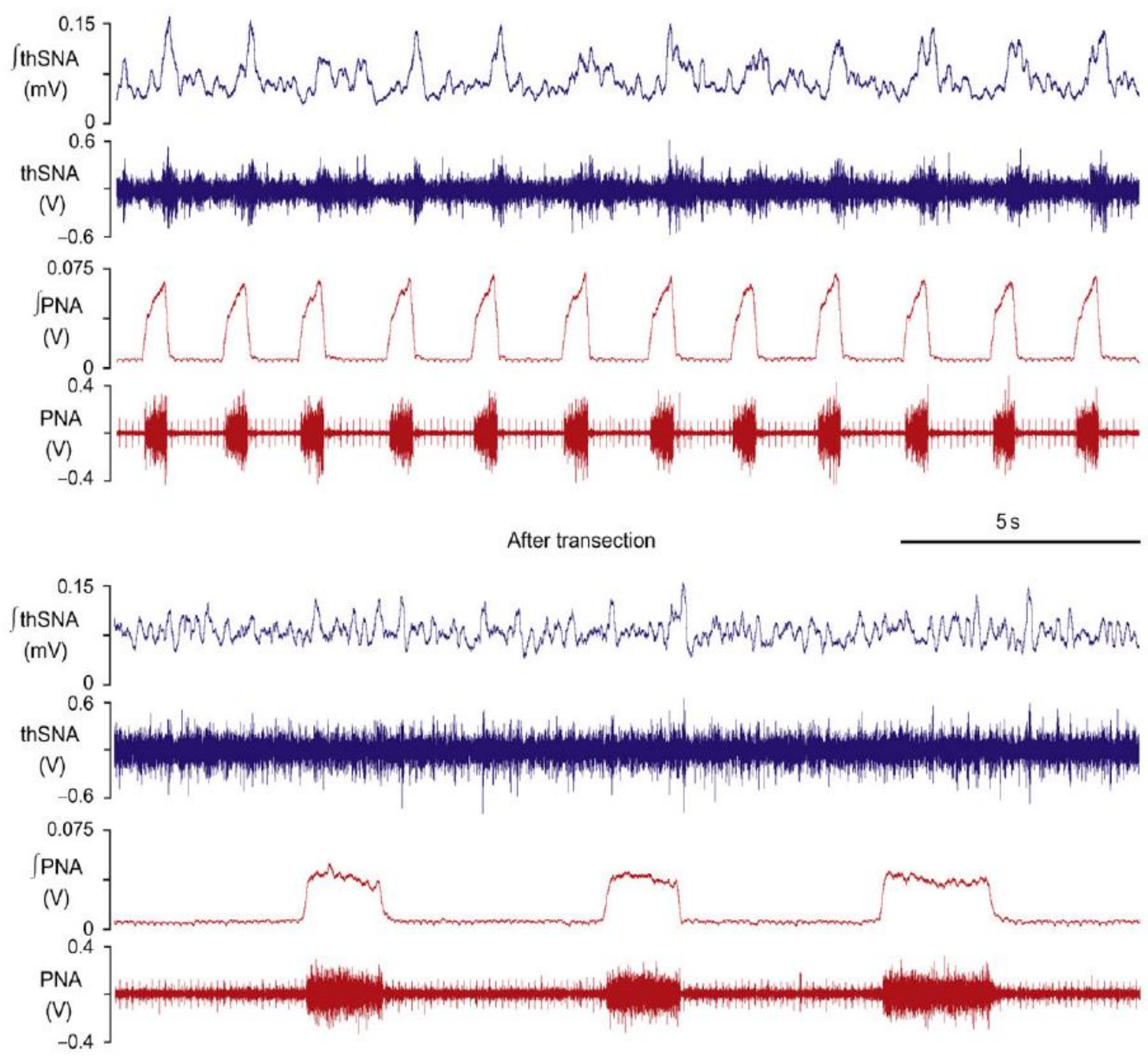

Fig. 12. Effects of pontomedullary transection on respirophasic modulation of thoracic sympathetic nerve activity. Mechanical transection between the metencephalon and myelencephalon abolishes respirophasic modulation of thoracic sympathetic (thSNA) nerve bursting in the in situ arterially-perfused preparation of the unanesthetized decerebrate juvenile rat. Upper panels: Thoracic sympathetic nerve bursting evidence late-inspiratory and postinspiratory accentuation during normoxic normocapnia in the presence of pontomedullary continuity. Prior to pontomedullary transection (intact pons; upper panels), integrated $\left(\int\right)$ and raw thoracic sympathetic nerve activity exhibit a clear respirophasic modulation. Lower panels: Encephalotomy between the metencephalon and myelencephalon abolishes or significantly attenuates respirophasic modulation of raw and integrated $\left(\int\right)$ thoracic sympathetic nerve activity. Top to bottom: integrated $\left(\int\right)$ and raw thoracic sympathetic nerve activity (thSNA); integrated $\left(\int\right)$ and raw phrenic nerve activity (PNA). Modified with permission from Fig. 1 of Molkov et al. (2014).

eral medullary GABAergic interneurons and another group conveying synaptic drive to metencephalic-dependent post-inspiratory units. Dynamically incrementing postinspiratory neuronal spiking frequency parallels correlated reductions of augmenting lateexpiratory neuronal spiking frequency. Barostimuli delivered during the expiratory epoch coordinately elicit resurgence of postinspiratory neuronal spiking frequency commensurate with dynamic reductions of discharge precluding waning activity and attenuating augmenting late-expiratory neuronal spiking, in essence resetting the expiratory epoch. An excitatory population of Bötzinger complex expiratory-related neurons, putatively glutamatergic, may convey direct axodendritic and axosomatic synaptic drive to rostral ventrolateral medullary neurons (Molkov et al., 2014). Bötzinger complex decrementing postinspiratory neurons are released from inhibitory modulation by preBötzinger complex decrementing early inspiratory neurons and rostral ventral respiratory group inspiratory neurons during early neural expiration (Smith et al., 2007), in effect coordinately amplifying neural expiratory activity and attenuating respirophasic modulation of sympathetic discharge (Baekey et al., 2010). Bötzinger complex decrementing postinspiratory neurons (see Ezure and Manabe (1988)) convey excitatory and/or inhibitory modulation to Bötzinger complex augmenting late expiratory neurons (Smith et al., 2009), preBötzinger complex preinspiratory, pre-inspiratory-inspiratory phase-spanning, and decrementing early-inspiratory units (Molkov et al., 2010), and rostral ventrolateral medullary presympathetic cells (Molkov et al., 2014; 
Moraes et al., 2012c; Sun et al., 1997).In contrast, Bötzinger complex augmenting late-expiratory neurons making direct synaptic contacts with adjacent rostral ventrolateral medullary cells (Moraes et al., 2012c; Sun et al., 1997) augment presympathetic bulbospinal neuronal spiking during late expiration, constituting a chief mechanism mediating sympathorespiratory coupling and strengthening the same in response to hypercapnia (Molkov et al., 2014) and acute and chronic intermittent hypoxia (Moraes et al., 2014b, 2012b; Zoccal et al., 2007, 2009a, 2008).

\section{Signal analysis \\ 12.1 Overview}

The signal analysis seeks to generate static or dynamic timefrequency representations of unitary, multi-unitary, or population firing behavior and inter-ensemble synchrony in the frequency and/or time domains (Ghali and Marchenko, 2013; Marchenko et al., 2012; Preobrazhenskii and Iarovitskii, 1963). Oscillations generated by the breathing generator, sympathetic oscillators, and cardiovagal premotoneurons interact nonlinearly and dynamically (Christakos et al., 1991; Dittler and Garten, 1912; Julien, 2006; Wyss, 2003). Nonlinear interactions occur amongst preganglionic sympathetic neurons supplying the heart with oscillatory waves conferred by mechanical ventilatory fluctuations in vagotomized unanesthetized decerebrate cats (Montano et al., 1992) and slow and fast components of postganglionic sympathetic discharge in pentobarbital-anesthetized sinoaortic denervated vagotomized cats (Zhong et al., 1998). Linear techniques, including cross-spectral density and coherence analyses, adequately characterize nature and stringency of synchronization amongst waves, thus informing the probability of coordinate genesis by a common oscillatory mechanism or local coupling mechanisms at the level of the individual oscillators (Marchenko et al., 2012). However, these techniques suffer from providing inadequate descriptions of the precise nature and causality of oscillatory interactions. Thus, the development and use of nonlinear techniques may inform and improve an understanding of dynamics and interactions of these oscillations according to a precise and elegant mathematical formalism (Hesse et al., 2003; Smirnov et al., 2008; Sysoev et al., 2016; Sysoeva et al., 2014).

The signal analysis permits the faithful conduct of coherence analysis between individual neuronal activity with en masse continuous neural efferent discharge (Marchenko et al., 2012). While most studies have correlated neural waveforms to determine coupling amongst the sympathetic and respiratory activities utilizing classic techniques (Christakos et al., 1991), a plethora of exploitable techniques abound in the literature. They may prove to be of significant potential in describing the behavior and character of these interactions. We point to the classic techniques of Fourier transformation, cross-correlation, autocorrelation, spectral power determination, cross-spectral density coherence, and dynamic time-frequency representations of coherence (Gavrovska et al., 2014; Ghali and Marchenko, 2013; Marchenko et al., 2012). We concurrently detail more intricate techniques which may prove useful in precisely determining wave coupling, including nonlinear correlation (Pijn et al., 1992), partial directed coherence (Baccalá and Sameshima, 2001), structure determination (Baccalá and Sameshima, 2001), linear (Hesse et al., 2003) and nonlinear Granger causality (Sysoeva et al., 2014), transfer entropy (Schreiber, 2000), and phase modeling (Smirnov et al., 2008), among other nuanced and specific techniques and approaches (Sysoev et al., 2016).

\subsection{Coherence between motoneuron and neurogram recordings}

To perform correlation or coherence analysis between two signals, the signals must remain continuous (Marchenko et al., 2012). This requirement precludes performing coherence analysis between unitary neuronal spiking with neural efferent output. Accordingly, we previously presented a study seeking to determine the presence of high-frequency oscillations in the spectra of individual phrenic motoneuronal recordings correlated with similar spectra observed in the Fast Fourier transforms of monopolar phrenic nerve recordings and provided evidence indicating high-frequency phrenic motoneurons oscillating at this frequency directly contribute to this spectral band of rhythmic activity in the phrenic neurogram (Marchenko et al., 2012). To achieve these goals, we needed to identify phrenic motoneurons (PhMNs) spiking at these rates and correlate this discharge with population activity in efferent phrenic nerve $(\mathrm{PhN})$ recordings (Marchenko et al., 2012). Phrenic motoneuron action potentials were derived at peak electropositive membrane voltage using Spike2CED software, and this unitary activity transformed into a continuous semicosine wave utilizing custom-designed scripts (Marchenko et al., 2012). We subsequently normalized the inspiratory epoch from 0 to 1.0 and averaged phrenic motoneuronal and neurogram activities gated by phrenic inspiratory burst onset and offset (Marchenko et al., 2012).

A magnified view of the phrenic burst reveals a difficult to appreciate, though discernible distinction, during the transition between inspiratory and postinspiratory epochs, with an apparent, though subtle segmentation of the burst components by a narrow inter-burst segment containing a paucity of oscillations (Ghali, 2015). Less magnified views of the phrenic burst effectively identify the border separating inspiratory and postinspiratory phases by a transition from augmenting to decrementing spatiotemporal dynamics of activity (Ghali, 2015). This strategy permitted us to determine coherence between the two waves with respect to discrete times within the inspiratory epoch and frequencies of observed phrenic motoneuronal and nerve discharge by performing smoothed pseudo-Wigner Ville distribution (SPWVD) time-frequency representation (TFR) coherence analysis between the phrenic motoneuron semi cosine wave and efferent phrenic nerve discharge using custom-designed MATLAB scripts (Gavrovska et al., 2014; Ghali and Marchenko, 2013; Marchenko et al., 2012; Pereira de Souza Neto et al., 2001; Tağluk et al., 2005). To perform smoothing in the time and frequency domains, we applied the previously described moving kernel function (Marchenko et al., 2012).

\section{Perspectives and significance}

Several mechanisms conspire to emergently generate sympathorespiratory, parasympathorespiratory, and sympathoparasympathetic coupling at rest and during hypercapnic and hypoxic conditions (Molkov et al., 2017; Moraes et al., 2014a, 2012a, 2014b, 2012b,c; Zoccal, 2015; Zoccal et al., 2007, 2009a; Zoccal and Machado, 2011; Zoccal et al., 2009b, 2008). Common inputs may generate Autonomorespiratory coupling conveyed to propriobulbar interneuronal microcircuit oscillators constituting the breathing pattern generator, sympathetic oscillators, and ambiguual cardiovagal premotoneurons, i.e., RTN/pFRG, see Abdala et al. (2009), coordinate and heterologous regulation by common ascending inputs relaying peripheral oscillatory baroreceptor, chemoreceptor, and pulmonary stretch receptor inputs (Takakura et al., 2007), and 
direct neurosynaptic interactions amongst overlapping propriobulbar interneuronal microcircuit oscillators of the constituent generators (i.e., Bötzinger complex and rostral ventrolateral medulla, see Moraes et al. (2012c) and Sun et al. (1997)). The presented set of conjectures and hypotheses carry equivalent empirical validity and naturally evolve the construction of an elegant model recapitulating and elucidating the mechanistic behavior of experimentally-evident autonomorespiratory coupling (Molkov et al., 2014).

Bötzinger complex decrementing post-inspiratory (Ezure and Manabe, 1988) and augmenting late-expiratory (Jean, 1992) neurons overlap with rostral ventrolateral medullary catecholaminergic, and glutamatergic presympathetic bulbospinal units (Ghali, 2017a, 2018; Guyenet et al., 2018; Guyenet, 2006) and pre-Bötzinger complex preinspiratory, preinspiratory-inspiratory phase spanning, and decrementing early inspiratory units (Molkov et al., 2017; MorgadoValle and Beltran-Parrazal, 2017) overlap with caudal ventrolateral medullary GABAergic interneurons (Goodchild and Moon, 2009; Mandel and Schreihofer, 2006; Paxinos et al., 1985). Excitatory and inhibitory neurosynaptic interactions coupling these cells emergently generates and strengthens sympathorespiratory coupling (Marchenko et al., 2016; Molkov et al., 2014). Thus, the relative balance of excitatory and inhibitory drive conveyed by, and synaptic contacts with, intermingled Bötzinger complex glycinergic decrementing postinspiratory, glutamatergic decrementing postinspiratory, and GABAergic augmenting late expiratory propriobulbar neuronal circuitry may chiefly determine the extent of respirophasic modulation of rostral ventrolateral medullary neuronal spiking (Dergacheva et al., 2010; Farmer et al., 2016). Dynamic changes of Bötzinger complex postinspiratory neuronal spiking frequency (see Ezure and Manabe (1988)) modifies rostral ventrolateral medullary neuronal spiking frequency during the postinspiratory and late expiratory epochs of breathing (see Moraes et al. (2012c) and Sun et al. (1997)). We suggest these direct neurosynaptic interactions and their proximally-related coordinately coupling contribute to emergently generating preferential late-expiratory bursting synchronized across abdominal and thoracic sympathetic neural efferent bursting during hypercapnia (Molkov et al., 2014) and acute and chronic intermittent hypoxia (Moraes et al., 2014b, 2012b; Zoccal et al., 2007, 2009a, 2008). During normocapnic normoxia, Bötzinger complex augmenting late-expiratory units restrict Bötzinger complex decrementing postinspiratory spiking (see classic models of Molkov et al. (2017, 2014) and Smith et al. (2007, 2009)), disinhibiting rostral ventrolateral medullary spiking during the postinspiratory epoch (Moraes et al., 2012c; Sun et al., 1997) and conferring postinspiratory preferential modulation of rostral ventrolateral medullary cells across the majority of studies (Koshiya and Guyenet, 1995; Mandel and Schreihofer, 2006; Numao et al., 1987; Seals et al., 1993; St. Croix et al., 1999). Excitatory and/or inhibitory populations of pre-Bötzinger complex post-inspiratory neurons (Ausborn et al., 2018) may contribute to sympathorespiratory coupling amongst rostral ventrolateral medullary presympathetic neurons through modulation of GABAergic and/or glutamatergic interneurons residing within the caudal ventrolateral medulla.

Hypercapnic stimulation of Phox $2 b$ transcription factorexpressing chemosensitive glutamatergic retrotrapezoid nucleus neurons (Guyenet et al., 2019) provides tonic drive to Bötzinger complex augmenting late-expiratory units (Molkov et al., 2017), which convey reciprocal inhibitory axodendritic and axosomatic synaptic drive to Bötzinger complex decrementing post-inspiratory neurons, according to the majority of modelers (Molkov et al., 2017; Smith et al., 2009). Experimental excitatory stimulation of propriobulbar interneuronal microcircuits oscillators residing within the Kölliker-Fuse and medial parabrachial nuclei variably generate excitation or inhibition of rostral ventrolateral medullary presympathetic neuronal activity (Baekey et al., 2010; Molkov et al., 2011; Smith et al., 2009). Accentuation or attenuation of either of these sets of Bötzinger complex expiratory populations coordinately modulates the discharge of rostral ventrolateral medullary neuronal spiking directly and through coordinate synaptic inputs from the originate drivers (Moraes et al., 2012c; Sun et al., 1997). Bötzinger complex and rostral ventrolateral medullary propriobulbar interneuronal microcircuit oscillators receive coordinate synchronizing axodendritic and/or axosomatic synaptic drive deriving from higher-order structures, including retrotrapezoid nucleus neurons (Guyenet et al., 2019), parafacial respiratory group pre-inspiratory cells (Onimaru et al., 2018), Kölliker-Fuse, medial, and lateral parabrachial nuclei (Dutschmann and Herbert, 2006; Mörschel and Dutschmann, 2009), and periaqueductal gray matter (Martin and Booker, 1878; Subramanian and Holstege, 2014), among other structures distributed throughout the telencephalon, diencephalon, brainstem, and cerebellum (Horn and Waldrop, 1997). Oscillatory baroreceptor, chemoreceptor, and pulmonary mechanoreceptor stimuli relaying through nucleus tractus solitarius units (Marchenko and Sapru, 2000; Moreira et al., 2007; Rogers et al., 2000; Takakura et al., 2007) and ascending spinoreticular pathways may also coordinately modulate Bötzinger complex and rostral ventrolateral medullary propriobulbar interneuronal microcircuit oscillator discharge.

Caudal ventrolateral medullary GABAergic interneuronal spiking imposes respirophasic modulation upon rostral ventrolateral medullary presympathetic neurons (Mandel and Schreihofer, 2006). PreBötzinger complex inhibitory decrementing early inspiratory neurons (see Molkov et al. (2017) and Smith et al. (2009)) may generate inhibitory postsynaptic currents observed in rostral and caudal ventrolateral medullary cells (Molkov et al., 2014). Populations of preBötzinger complex units alternately expressing GABAergic (Ellenberger, 1999) and glycinergic (Morgado-Valle et al., 2010) neurochemical phenotypes. Analogously inhibitory axodendritic and axosomatic drive may contribute to generating fast synchronous oscillatory coupling and synchrony, clearly manifest in phrenic nerve discharge spectra of the in situ arterially-perfused preparation of the unanesthetized decerebrate juvenile rat and in vivo unanesthetized decerebrate preparations of the adult rat (Ghali and Marchenko, 2013; Marchenko and Rogers, 2007; Marchenko et al., 2012; Marchenko and Rogers, 2006a,b). Marchenko and Rogers (2009) provide precedence indicating the critical contribution of fast inhibitory neurosynaptic transmission in modulating the properties of fast sympathetic rhythms. Phrenic nucleus microinjections of gabazine or strychnine elicit severe perturbations of fast oscillatory synchrony in population phrenic nerve motor output Marchenko and Rogers (2009). Strychnine microinjections in the phrenic nucleus robustly amplified phrenic nerve activity during the postinspiratory phase, suggesting local glycinergic mechanisms restrict post-inspiratory activity in phrenic neural efferent discharge. However, the effects of gabazine were less phase-restricted in amplifying phrenic nerve activity across the respiratory cycle. Interactions at the level of nucleus tractus solitarius units (Jean, 1992) inform novel mechanisms of sympathorespiratory and parasympa- 


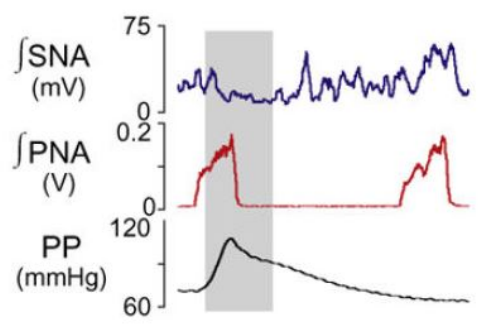

B

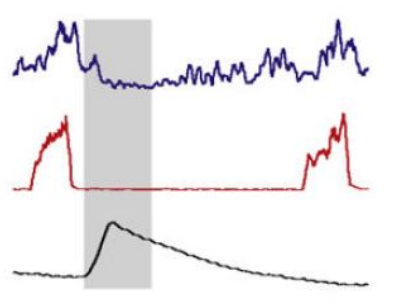

Expiration
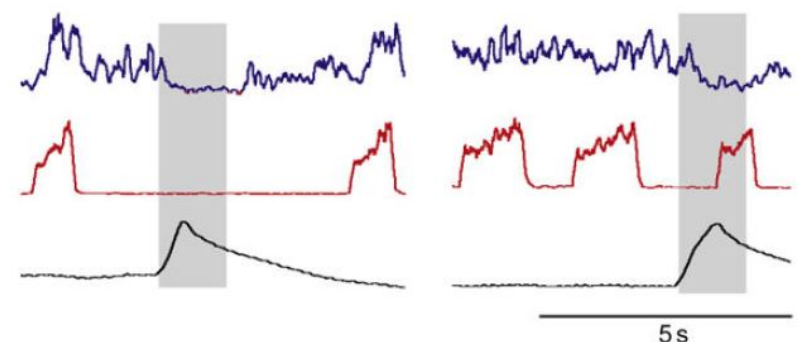

After pons removal

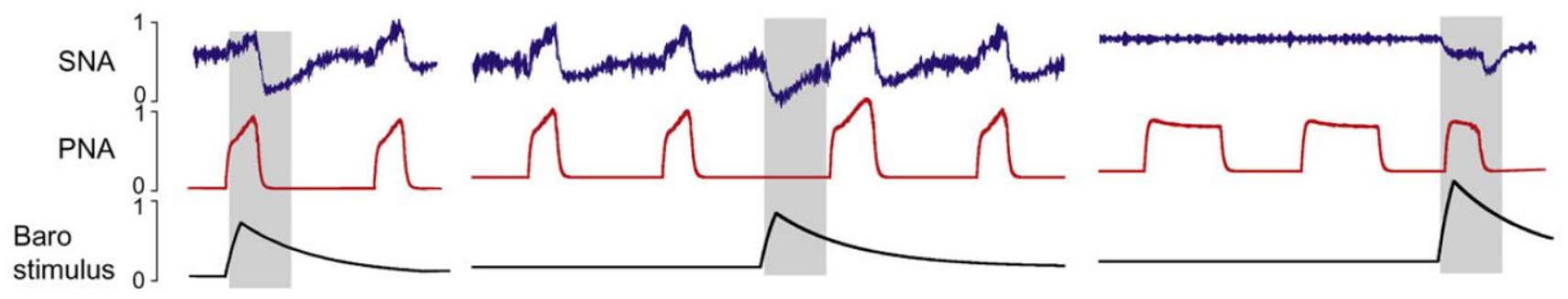

Fig. 13. Dynamic changes of arterial pressure magnitude elicit coordinate modification of phrenic and sympathetic neural bursting. A: Barostimuli applied during the inspiratory, postinspiratory, or late expiratory epochs in the in situ arterially-perfused unanesthetized decerebrate juvenile rat elicit differential effects upon neural breathing pattern. Barostimuli delivered during the inspiratory epoch failed to modify breathing rhythm or burst parameters. However, barostimuli applied during the postinspiratory, and late-expiratory epochs elicited robust amplification of expiratory epoch duration. Following mechanical transection separating the metencephalon from the myelencephalon, a barostimulus applied during late expiration contracted apneustic inspiratory bursts in the phrenic nerve discharge. Barostimuli most powerfully elicits reductions of sympathetic neural efferent activity when applied during inspiration, putatively consequent to preferentially inspiratory accentuation of sympathetic neural bursting during resting eucapnic hyperoxia and reduced excitatory synaptic drive from the glutamatergic excitatory population of Bötzinger complex decrementing postinspiratory neurons. $\int$ SNA, integrated sympathetic nerve activity ( $\mathrm{mV}$, millivolts); $\int$ PNA, integrated phrenic nerve activity (V, Volts); PP, perfusion pressure (mmHg); Timescale bar is shown in the lower right-hand corner (5 seconds). B: Computational model simulation of baroreceptor stimulus applied during inspiratory and expiratory epochs in preparations with theoretically preserved pontomedullary continuity intact model and during the inspiratory epoch following removal of the metencephalic compartment. The effects recapitulate empirically-observed respirophasic-variant influences of oscillatory baroreceptor inputs upon the breathing pattern. A simulated barostimulus presented during the inspiratory epoch with preserved pontomedullary continuity negligibly modifies breathing rhythm parameters, though effectively reduces sympathetic neural bursting. A simulated barostimulus applied during the late expiratory epoch elicits modest enhancement of expiratory epoch duration with reflex augmentation of amplitude and duration of ensuing inspiratory bursts coordinately with attenuation of sympathetic neural activity. Reflex augmentation of inspiratory bursting succeeds expiratory facilitation elicited by a barostimulus applied during late expiration, putatively resulting from recovery and resetting of phrenic motoneuronal membrane voltage trajectories and replenishing of neuronal metabolic adenosine triphosphate stores, permitting successively conveyed bulbospinal axodendritic and axosomatic synaptic drive conveyed from rostral ventral respiratory group augmenting inspiratory neurons to elicits early-inspiratory high-frequency oscillations exhibiting more robust coherence and synchrony amongst the population of phrenic motoneurons. Removal of the metencephalic compartment abolishes respirophasic modulation of sympathetic neural activity in the model, with barostimuli delivered during inspiration contracting apneustic inspiratory epochs and reducing sympathetic neural activity. Barostimuli applied during inspiration, and late expiration elicits similar reductions in sympathetic neural activity in preparations with preserved pontomedullary continuity. SNA; sympathetic nerve activity (amplitude normalized from 0 to 1); PNA, phrenic nerve activity (amplitude normalized from 0 to 1). Modified with permission from Fig. 3 of Molkov et al. (2014).

thorespiratory coupling. Oscillatory baroreceptor inputs transduced by pressure-sensitive receptors exhibiting highest density within the carotid sinus and aortic arch, though diffusely distributed throughout the internal carotid (Toorop et al., 2013), vertebral (Agadzhanian and Kupriianov, 2008; Kupriyanov, 2009), and subclavian arteries (Chevalier-Cholat and Friggi, 1977, 1976; Kidd et al., 1974) relays centrally to neurons residing within the medial and interstitial divisions of the nucleus tractus solitarius covarying dynamically with changes of arterial pressure magnitude (Rogers et al., 2000). Spatiotemporal bursting dynamics of integrated baroreceptor discharge frequency assumes the form of a sine wave. Oscillatory baroreceptors inputs emergently generate sympathorespiratory coupling by modulating overlapping Botzinger complex and rostral ventro- lateral medullary propriobulbar interneuronal microcircuit oscillators (Moraes et al., 2012c; Sun et al., 1997) through GABAergic caudal ventrolateral medullary interneurons (Guyenet et al., 2019; Guyenet, 2006; Mandel and Schreihofer, 2006).

Mechanosensitive pulmonary stretch receptors residing within the alveolar interstitium may be electrophysiologically segregated into slow (Fenik, 1992; Preobrazhenskil and Fenik, 1989) and fast (Zhdanov, 1984) adapting types of stretch receptors, with discharge frequency nonlinearly covarying according to an absolute, or static or dynamic rate of change of, pulmonary stretch (Preobrazhenskiı̌ and Fenik, 1989). Slow adapting pulmonary stretch receptors chiefly sensitive to the absolute magnitude of stretch generate com- 
plex spike trains during the phrenic burst (Chen et al., 2011, 2010, 2008; Marchenko and Rogers, 2007b), compared with fast adapting pulmonary stretch receptors, which possess rapid desensitization kinetics and are thus specifically tuned to detecting the onset and offset of the inspiratory epoch (Zhdanov, 1984). Spike trains of these receptors relay centrally via pulmonary stretch mechanosensitive vagal afferents to pump cells chiefly residing in the ventrolateral division of the nucleus tractus solitarius (Moreira et al., 2007; Takakura et al., 2007). Higher-order nucleus tractus solitarius units may alternately receive the monomodal or multimodal convergent somatodendritic synaptic drive from afferents relaying oscillatory inputs from baroreceptors (Rogers et al., 2000), chemoreceptors (Takakura et al., 2007), or pulmonary stretch mechanoreceptors (Li et al., 1999b,a; Moreira et al., 2007; Takakura et al., 2007). Rises of dynamic arterial pressure magnitude occurring during the inspiratory epoch elicit multiplicative amplification of neuronal spiking frequency of multimodal nucleus tractus solitarius units, contrasted with rises of dynamic arterial pressure magnitude during expiration which elicit comparatively more attenuated augmentations of NTS neuronal spiking frequency (Rogers et al., 2000).

These findings collectively mechanistically indicate oscillatory mechanosensory pulmonary stretch spike cell trains heterosynaptically gates oscillatory baroreceptor inputs to NTS units. Reciprocally, oscillatory baroreceptor spike cell trains heterosynaptically gates oscillatory pulmonary stretch inputs to NTS units (Baekey et al., 2010). More simply stated, neural inspiratory activity gates baroreceptor oscillations and dynamic arterial pressure magnitude gates pulmonary stretch receptor spike trains conveyed to nucleus tractus solitarius units (Baekey et al., 2010). Glutamatergic barosensitive nucleus tractus solitarius units convey excitatory axodendritic and axosomatic synaptic drive to caudal ventrolateral medullary GABAergic propriobulbar interneurons and may heterosynaptically blunt the discharge of glutamatergic chemosensitive retrotrapezoid nucleus units (Guyenet, 2006), conferring oscillatory baroinhibition of rostral ventrolateral medullary presympathetic unitary spiking (Mandel and Schreihofer, 2006). By this mechanism, baroreceptor oscillations modulate the sympathetic neural efferent output (Diedrich et al., 2009; Ghali, 2017a, 2018; Guyenet et al., 2019). Critically, barosensitive nucleus tractus solitarius unitary spiking coordinately influences cardiovagal premotoneuronal spiking within the dorsal motor nucleus of the vagus and nucleus ambiguus innervating dendrites and somata of postganglionic parasympathetic cholinergic neurons in the cardiac plexus (Eckberg, 2003), accordingly modulating sinoatrial action potential frequency, atrioventricular conductivity, and myocardial contractility. Oscillatory fluctuations of arterial pressure, thus confer an oscillatory fluctuation upon the cardiac interval (Rogers et al., 2000), influencing spectral variability of dynamic arterial pressure magnitude.

A host of mechanisms reflecting the overall state and condition of the breathing generator, sympathetic oscillators, and ambiguual cardiovagal premotoneurons impose respirophasic modulation upon cardiovagal premotoneuronal spiking (Dergacheva et al., 2010; Farmer et al., 2016). The Bötzinger complex contains decrementing postinspiratory neurons exhibiting a predominantly glycinergic neurochemical repertoire (Ezure and Manabe, 1988), though a few of these units also exhibit a glutamatergic phenotype (Molkov et al., 2017; Smith et al., 2007). Bötzinger complex glycinergic and glutamatergic decrementing postinspiratory modulate cardiovagal premotoneuronal membrane voltage trajectories during the early expiratory epoch. The relative balance and weighing of synaptic inputs conveyed by these cells determine whether afferent influences generate predominantly expiratory facilitation or inhibition of efferent cardiovagal premotoneurons residing within the nucleus ambiguus and dorsal motor vagal nucleus (Dergacheva et al., 2010; Farmer et al., 2016). Direct synaptic drive conveyed by Bötzinger complex GABAergic cells to ambiguual cardiovagal premotoneurons (Dergacheva et al., 2010; Farmer et al., 2016) may represent a parallel mechanism generating attenuation of cardiovagal premotoneuronal tone and reciprocal sinoatrial accelerations.

Bötzinger complex decrementing postinspiratory neurons coordinately receive excitatory axodendritic and axosomatic drive from Kölliker-Fuse and medial parabrachial nucleus propriobulbar units (Dutschmann and Herbert, 2006; Mörschel and Dutschmann, 2009) and monosynaptic direct and polysynaptic indirect modulation conferred by monomodal or multimodal nucleus tractus solitarius units (Molkov et al., 2017; Smith et al., 2007) (Fig. 5). Bötzinger complex decrementing postinspiratory neurons modulate normal triphasic eupnea by segmenting the expiratory epoch through reciprocal inhibitory neurosynaptic interactions with Bötzinger complex augmenting late-expiratory neurons and via the provision of prominent inhibitory modulatory influences upon preBötzinger complex preinspiratory, preinspiratory inspiratory phase spanning and decrementing early inspiratory neurons (Molkov et al., 2017; Rybak et al., 2014; Smith et al., 2007). The direct overlap of the Bötzinger complex with rostral ventrolateral medullary propriobulbar and bulbospinal circuitry provides a neuroanatomic substrate generating common sympathorespiratory coupling and crossmodal modulation amongst these oscillatory zones (Moraes et al., 2014a,b).

According to the described neuroanatomic organization and patterns of electrophysiological interactions, any peripheral stimulus or set of influences modulating nucleus tractus solitarius unitary spiking frequency (Chen et al., 2011; Moreira et al., 2007; Rogers et al., 2000; Takakura et al., 2007) may coordinately modulate cardiovagal premotoneuronal (Bishop, 1974; Lindsey et al., 1998) and rostral ventrolateral medullary presympathetic unitary spiking (Moraes et al., 2012c; Sun et al., 1997) through axodendritic and axosomatic synaptic drive conveyed to Bötzinger complex neurons (Molkov et al., 2014). However, the specific type of stimulus modulating nucleus tractus solitarius neuronal spiking differential influences pattern of modulation conveyed upon propriobulbar interneuronal microcircuit oscillators generating breathing, sympathetic activity, and cardiovagal premotoneuronal discharge (Baekey et al., 2010; Lindsey et al., 1998). The magnitude and dynamic rates of changes of alveolar stretch detected by slow and fast adapting pulmonary stretch receptors (Chen et al., 2011) conveyed centrally through vagal afferents to the ventrolateral and interstitial divisions of the nucleus tractus solitarius (Marchenko and Sapru, 2000) commensurately modulate Bötzinger complex decrementing postinspiratory and augmenting late expiratory neuronal spiking to disfacilitates inspiration and enhance expiration (Molkov et al., 2017) and rostral ventrolateral medullary presympathetic units to generate sympathoinhibitory effects via the soi-disant sympathetic arm of the Hering Breuer reflex (Guyenet, 2006) and sinoatrial acceleration through disfacilitation of cardiovagal premotoneurons, consequent to inhibitory axodendritic and axosomatic modulation by Bötzinger complex GABAergic propriobulbar interneuronal microcircuit os- 


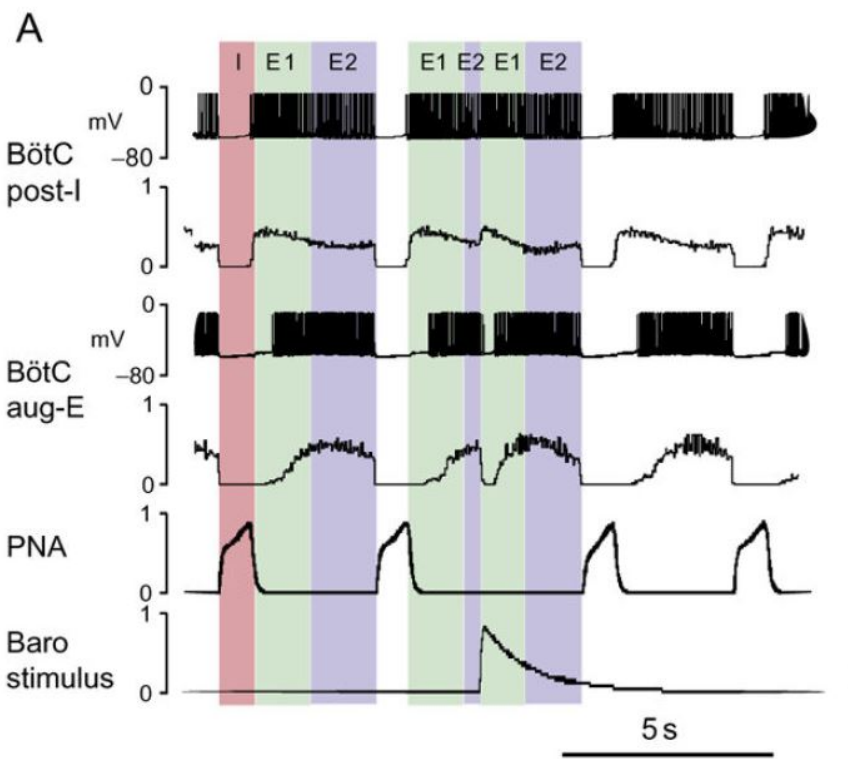

B

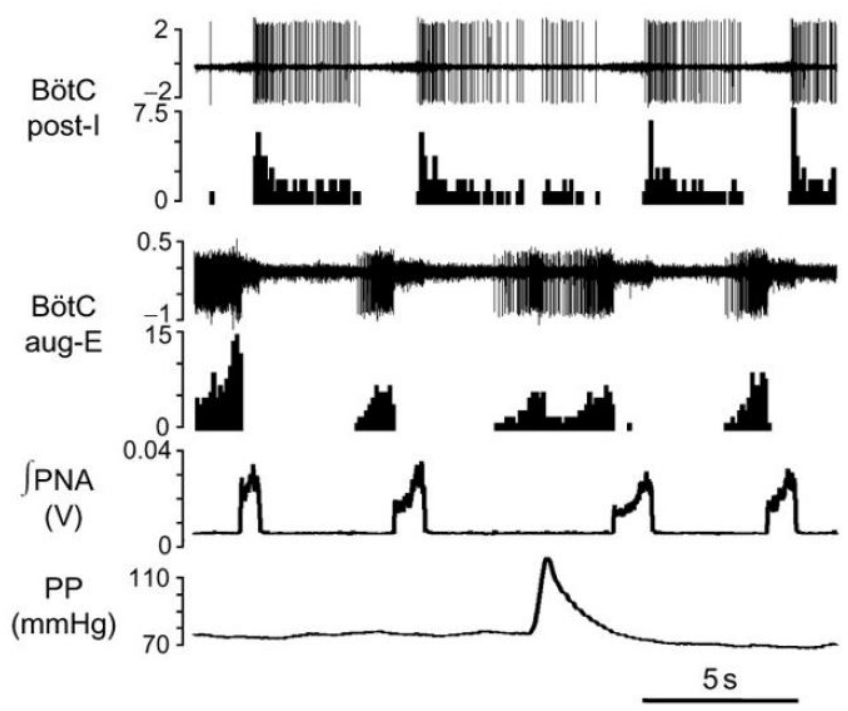

Fig. 14. Mechanism mediating resetting of expiration elicited by transient barostimuli. A: Computational modeling prediction of Bötzinger complex postinspiratory neuronal and phrenic nerve discharge to a barostimulus applied during the late expiratory epoch. Bötzinger complex post-inspiratory and augmenting late-expiratory neuron membrane voltage trajectories (first and third traces) and action potential spiking among both neuronal populations (second and fourth traces) are depicted. Shaded intervals within segregable respiratory epochs highlight inspiratory, early expiratory (E1), and late expiratory (E2) epochs. Barostimulation resets expiration by activating populations of Bötzinger complex postinspiratory units neurosynaptically inhibiting Bötzinger complex augmenting late-expiratory cells. Delivery of a barostimulus amid late expiration resurrects augmenting expiratory neuronal spiking and elicits a second complete expiratory effort. B: Extracellular recordings (first among each pair of upper and middle traces) and unitary spiking histograms (second among each pair of upper and middle traces) of Bötzinger complex decrementing post-inspiratory (upper pair of traces) and augmenting late expiratory (middle pair of traces) neurons. Integrated phrenic nerve activity ( $\int$ PNA) and perfusion pressure (PP) are successively depicted in the lower pair of traces. Barostimuli delivered during late expiration generate expiratory resetting in the in situ arterially-perfused preparation of the unanesthetized decerebrate juvenile rat. Barostimuli delivered during the late-expiratory epoch in the in situ preparation of the unanesthetized decerebrate juvenile rat enhances Bötzinger complex post-inspiratory neuronal spiking frequency and reduce Bötzinger complex augmenting late-expiratory activity, recapitulating model predictions. Modified with permission from Fig. 4 of Molkov et al. (2014).

cillators (Fenik, 1992; Preobrazhenskiĭ and Fenik, 1989; Zhdanov, 1984; Zhdanov et al., 1989).

Chemosensory stimulation of commissural nucleus tractus solitarius units coordinately enhances Bötzinger complex expiratory neuronal discharge and rostral ventrolateral medullary presympathetic unitary activity (Michelini, 1994; Moreira et al., 2007; Rogers et al., 2000; Schwaber et al., 1993; Takakura et al., 2007). Accordingly, hypercapnia or acute or chronic intermittent hypoxia enhances glutamatergic excitatory and GABAergic inhibitory modulation conveyed to dorsal medullary cardiovagal premotoneurons (Dergacheva et al., 2010; Farmer et al., 2016) permitting sinoatrial acceleration elicited by sympathoexcitation (see Guyenet et al. $(2018,2019))$ to support chemoreceptor-driven amplifications of myocardial output and dynamic arterial pressure magnitude. Factors influencing baroreflex gain and sensitivity (see Rogers et al. (2000)) may modify patterns of modulation of Bötzinger complex decrementing post-inspiratory and augmenting late-expiratory neurons by chemosensitive commissural nucleus tractus solitarius neuronal synaptic inputs. Chemosensitive glutamatergic retrotrapezoid nucleus units convey diffuse tonic excitatory drive to the Bötzinger complex, chiefly augmenting late-expiratory neurons, and receive prominent GABAergic modulatory synaptic drive deriving from nucleus tractus solitarius pump cells (Guyenet et al., 2019). These pathways constitute a network mechanism permit- ting pulmonary stretch receptor-mediated blunting of chemosensory amplification of neural respiratory output, relaying to glutamatergic retrotrapezoid nucleus units via nucleus tractus solitarius pump cells (Takakura et al., 2007) and conjecturally powerfully restricting the preinspiratory component of hypoglossal bursting in the presence of preserved vagal continuity (Ghali, 2015, 2019c; Ghali and Marchenko, 2016b).

Peripheral chemoreceptors constituted glomus cells exquisitely sensitive to hypercapnia, and moderate and severe levels of hypoxemia chiefly utilize purinergic signaling (Baby et al., 2006) and convey stimulus mediated modifications of neuronal spiking frequency centrally to chemosensitive glutamatergic commissural nucleus tractus solitarius neurons (Zhang et al., 2011). Glutamatergic chemoreceptor neurons distributed throughout the retrotrapezoid nucleus and parafacial respiratory group complex (Guyenet et al., 2019; Onimaru et al., 2018), nucleus tractus solitarius (Nichols et al., 2008), raphé group of nuclei (Sobrinho et al., 2014), preBötzinger complex, and fastigial nucleus (Hernandez et al., 2004) are chiefly sensitive to elevations of carbon dioxide tension or hydrogen ion concentration, with a few zones coordinately exhibiting hypoxic sensitivity (e.g., preBötzinger complex, fastigial nucleus). Hypercapnia and hypoxia elicit and amplify discharge of peripheral chemoreceptor glomus cells (Zoccal, 2015) and chemosensitive bulbar units (Guyenet et al., 2019) conveying coordinate synaptic 
drive to breathing generators (Marchenko et al., 2016; Molkov et al., 2014; Zoccal and Machado, 2011; Zoccal et al., 2008), sympathetic oscillators (Gilbey et al., 1995), and cardiovagal premotoneurons (Bishop, 1974; Lindsey et al., 1998), crossmodally sensitizing each other's discharge (Blain et al., 2010) and conveying augmented coherent neuronal spiking through propriobulbar interneuronal paucisynaptic interactions (Molkov et al., 2014).

Intravenous sodium chloride administration coordinately enhanced phrenic nerve bursting frequency and thoracic sympathetic neural burst amplitude in decorticate Holtzman rats, both effects of which were abolished by precollicular transection, supporting paraventricular and supraoptic nuclei mediate the effects (da Silva et al., 2019). Carotid body removal prevented the enhancement of thoracic sympathetic nerve activity elicited by intravenous administration of sodium chloride, though failed to prevent tachypnea, supporting carotid body glomus cells may represent effective osmoreceptors (da Silva et al., 2019). Peripheral and central chemoreceptor driven augmentation of the respiratory, central pattern generator and sympathetic oscillators becomes synchronized and commonly coupled through coordinate and independent bulbobulbar, spinoreticular, and peripheral afferent inputs couple and synchronize central and peripheral chemoreceptor mediated augmentation of neuronal ensembles constituting the constituent generators, strengthened by direct interactions between overlapping propriobulbar interneuronal arrays constituting the respiratory rhythm and pattern generator, sympathetic oscillators, and cardiovagal premotoneurons(Molkov et al., 2017; Zoccal, 2015). These pathways constitute mechanisms generating autonomorespiratory coupling and crossmodal modulation amongst disparate oscillators and nuclei at rest during normoxic normocapnia, which becomes amplified by hypercapnia and acute and chronic hypoxia (Figs. 1-5) (Molkov et al., 2017; Zoccal, 2015). Sympathorespiratory, parasympathorespiratory, and sympathoparasympathetic coupling may further be subject to modulation by cardiovascular and respiratory muscle training, putatively mediated by amplifying the cardiovagal sinoatrial decelerator tone, evidenced in powerful modifications of the cardiovascular spectral variabilities (de Abreu et al., 2019).

\section{Conclusions}

The respiratory rhythm (Anderson et al., 2016; Anderson and Ramirez, 2017; Ghali, 2019c; Morgado-Valle and Beltran-Parrazal, 2017; Ramirez and Baertsch, 2018) and pattern (Ghali, 2018; Marchenko et al., 2016; Ramirez and Baertsch, 2018; Smith et al., 2009), sympathetic oscillations (Ghali, 2017a, 2019b, 2018), and cardiovagal premotoneuronal activity (Baekey et al., 2010; Bishop, 1974) are generated by distinct, though overlapping, networks residing chiefly within the metencephalon and myelencephalon (Ghali, 2017a,b,c, 2019a,b; Marchenko et al., 2016; Molkov et al., 2017) and powerfully modulated by descending synaptic inputs from the cerebrum (Antal, 1985), hippocampus (Ruit and Neafsey, 1988), amygdala (Lacuey et al., 2019), thalamus (Ogundele et al., 2017), hypothalamus (Fukushi et al., 2019), midbrain (Ghali and Ghali, 2020), and cerebellum (Horn and Waldrop, 1997; Schmid et al., 1988; Subramanian and Holstege, 2014). The widespread presence of autonomorespiratory coupling, manifest as baroreceptor and sympathetic modulation of neural respiratory activity (Baekey et al., 2010; Bishop, 1974; Lindsey et al., 1998), respirophasic modulation of sympathetic discharge (Haselton and Guyenet, 1989; Mandel and Schreihofer, 2006; McAllen, 1987; Molkov et al., 2017), and ambiguual cardiovagal premotoneurons (Dergacheva et al., 2010), and crossmodal modulation between sympathetic and parasympathetic outflows evidence coordinate inputs to, and direct interaction amongst and between, propriobulbar interneuronal microcircuit oscillators constituting the pattern generators mediating the neurogenesis of these activities and heterologous peripheral modulation of breathing, sympathetic oscillations, and cardiovagal premotoneurons by vagal afferents conveying oscillatory baroreceptor, chemoreceptor, and pulmonary stretch receptor inputs (Molkov et al., 2017; Zoccal, 2015). Sympathorespiratory and cardiorespiratory coupling thus critically requires integrity and functionality of the core metencephalomyelencephalic generator circuitry and elements diffusely distributed within the ventrolateral and dorsolateral metencephalic tegmentum, brainstem reticular formation, vagal inputs, and postinspiratory neurons (Fig. 5) (Molkov et al., 2017), elucidating mechanisms contributing to emergent genesis of autonomorespiratory coupling through studies utilizing contemporaneous respiratory and sympathetic neuronal (Terui et al., 1986) and peripheral sympathetic neural efferent recordings (St. Croix et al., 1999), directed lesioning (Dick et al., 2009; Molkov et al., 2011), stereotaxic localized pressure microinjections of pharmacological agonists and antagonists of excitatory and inhibitory neurotransmission and neuromodulation (Abdala et al., 2009; Moraes et al., 2012b), and cross-correlation, spectral, and coherence analyses of the recorded electrophysiological activities. While the alternating rhythmic discharge of inspiratory and expiratory activities of breathing neurally segmented into inspiratory, postinspiratory, and late expiratory epochs effectively serves a clear and identifiable functional purpose (Marchenko et al., 2016), the significance of rhythmic discharge in, and crossmodal modulation of, sympathetic oscillations, sympathetic neural efferent discharge, and ambiguual cardiovagal premotoneurons is not immediately obvious (Zoccal, 2015). Some have posited respirophasic modulation of the sympathetic neural output may contribute to and mediate coordinate changes in ventilation and vascular tone to contemporaneously optimize tissue oxygenation and perfusion during resting conditions and amplify vasoconstrictor arteriolar tone and amplitude of the microcirculatory vasomotion (Zoccal et al., 2009b). Accordingly, modeling studies have indicated interactions among the respiratory, central pattern generator, sympathetic oscillators, and parasympathetic nuclei may serve to robustly improve myocardial function (Ben-Tal et al., 2012). Further studies are thus necessary to more thoroughly elucidate functional utility and mechanisms underlying the genesis and functional utility of autonomorespiratory coupling (Molkov et al., 2017).

\section{Author contributions}

M.G.Z.G.: conception and design, acquisition of data, analysis and interpretation of data, drafting the article, and revising critically for intellectual content; approval of the final version of the manuscript.

\section{Ethics approval and consent to participate}

Compliance with ethical standards.

\section{Acknowledgments}

We appreciate the helpful comments from anonymous reviewers for improving the quality of this review. 


\section{Conflict of Interest}

We have no conflicts of interest to disclose.

Submitted: June 28, 2019

Revised: December 02, 2019

Accepted: December 04, 2019

Published: September 30, 2020

\section{References}

Abdala, A. P. L., Rybak, I. A., Smith, J. C., Zoccal, D. B., Machado, B. H., St-John, W. M. and Paton, J. F. R. (2009) Multiple pontomedullary mechanisms of respiratory rhythmogenesis. Respiratory Physiology \& Neurobiology 168, 19-25.

Adrian, E. D., Bronk, D. W. and Phillips, G. (1932) Discharges in mammalian sympathetic nerves. The Journal of Physiology 74, 115-133.

Agadzhanian, N. A. and Kupriianov, S. V. (2008) Baroreflexes originated in vertebral artery zones upon peripheral vein tonus, systemic arterial blood pressure, and external respiration. Rossiiskii Fiziologicheskii Zhurnal Imeni i.M. Sechenova 94, 661-669. (In Russian)

Alexander, N. and Cuir, M. D. (1963) Role of aortic and vagus nerves in arterial baroreflex bradycardia in rabbits. American Journal of PhysiologyLegacy Content 205, 775-780.

Anastasievich, R., Vasilenko, D. A., Kostiukov, A. I. and Preobrazhenskiū, N. N. (1974) Reticulofugal influences on interneurons distributed in the lateral portions of cat spinal cord gray matter. Neirofiziologiia 5, 525536. (In Russian)

Anderson, T. M., Garcia, A. J., Baertsch, N. A., Pollak, J., Bloom, J. C., Wei, A. D., Rai, K. G. and Ramirez, J. (2016) A novel excitatory network for the control of breathing. Nature 536, 76-80.

Anderson, T. M. and Ramirez, J. (2017) Respiratory rhythm generation: triple oscillator hypothesis. F1000Research 6, 139.

Anrep, G. V. and Segall, H. N. (1926) The central and reflex regulation of the heart rate. The Journal of Physiology 61, 215-231.

Anrep, G. V., Pascual, W. and Rossler, R. (1936) Respiratory variations of the heart rate. I. The reflex mechanism of the respiratory arrhythmia. Proceedings of the Royal Society London Series B, Biological Sciences 119, 191-217.

Antal, M. (1985) Termination areas of corticobulbar and corticospinal fibres in the rat. Journal Fur Hirnforschung 25, 647-659.

Ausborn, J., Koizumi, H., Barnett, W. H., John T. T., Zhang R., Molkov Y. I., Smith J. C. and Rybak I. A. (2018) Organization of the core respiratory network: Insights from optogenetic and modeling studies. PLoS Computational Biology 14, e1006148.

Babic, T. and Ciriello, J. (2004) Medullary and spinal cord projections from cardiovascular responsive sites in the rostral ventromedial medulla. The Journal of Comparative Neurology 469, 391-412.

Baby, S. M., Roy, A. and Lahiri, S. (2006) Role of mitochondria in the regulation of hypoxia-inducible factor-1alpha in the rat carotid body glomus cells. Histochemistry and Cell Biology 124, 69-76.

Barcroft, J. and Nisimaru, Y. (1932) Undulatory changes of blood-pressure. Journal of Physiology 74, 311-320.

Baccalá, L. A. and Sameshima, K. (2001) Partial directed coherence: a new concept in neural structure determination. Biological Cybernetics 84, 463-474.

Baekey, D. M., Dick, T. E. and Paton, J. F. R. (2008) Pontomedullary transection attenuates central respiratory modulation of sympathetic discharge, heart rate and the baroreceptor reflex in thein siturat preparation. Experimental Physiology 93, 803-816.

Baekey, D. M., Molkov, Y. I., Paton, J. F. R., Rybak, I. A. and Dick, T. E. (2010) Effect of baroreceptor stimulation on the respiratory pattern: Insights into respiratory-sympathetic interactions. Respiratory Physiology \& Neurobiology 174, 135-145.

Baev, K. V., Shapovalov, A. V., Preobrazhenskiı̌, N. N. and Esipenko, V. B. (1981) [Changes in the excitability of central endings of the superior laryngeal nerve following activation of the respiratory center]. Neirofiziologiia 12, 653-655. (In Russian)

Barman, S. M., Orer, H. S. and Gebber, G. L. (2005) Role of medullary excitatory amino acid receptors in mediating the $10-\mathrm{Hz}$ rhythm in sympathetic nerve discharge of cats. Brain Research 1049, 249-253.

Barman, S. M. (2020) 2019 Ludwig Lecture: Rhythms in sympathetic nerve activity are a key to understanding neural control of the cardiovascular system. American Journal of Physiology-Regulatory, Integrative and Comparative Physiology 318, R191-R205.

Barnett, W. H., Abdala, A. P., Paton, J. F. R., Rybak, I. A., Zoccal, D. B. and Molkov, Y. I. (2017) Chemoreception and neuroplasticity in respiratory circuits. Experimental Neurology 287, 153-164.

Barrès, C., Cheng, Y. and Julien, C. (2004) Steady-state and dynamic responses of renal sympathetic nerve activity to air-jet stress in sinoaortic denervated rats. Hypertension 43, 629-635.

Ben-Tal, A., Shamailov, S. S. and Paton, J. F. R. (2012) Evaluating the physiological significance of respiratory sinus arrhythmia: looking beyond ventilation-perfusion efficiency. The Journal of Physiology 590, 19892008.

Bezhenaru, I. S., Gokin, A. P., Zadorozhnyi, A. G. and Preobrazhenskii, N. N. (1973) Synaptic activation of thoracic spinal interneurons via reticulospinal pathways. Neirofiziologiia 4, 566-578. (In Russian)

Bishop, B. (1974) Carotid baroreceptor modulation of diaphragm and abdominal muscle activity in the cat. Journal of Applied Physiology 36, 12-19.

Blain, G. M., Smith, C. A., Henderson, K. S. and Dempsey, J. A. (2010) Peripheral chemoreceptors determine the respiratory sensitivity of central chemoreceptors to CO2. The Journal of Physiology 588, 2455-2471.

Bongianni, F., Mutolo, D., Cinelli, E., Pantaleo, T. (2010) Respiratory responses induced by blockades of GABA and glycine receptors within the Bötzinger complex and the pre-Bötzinger complex of the rabbit. Brain Research 1344, 134-147.

Breuer, J. (1868) Die Selbststeurung der Athmung durch den Nervus Vagus. Akademie der Wissenschaften Wien (II) 58, 909-937. (In German)

Brodie, T. G. and Russell, A. E. (1900) On reflex cardiac inhibition. The Journal of Physiology 26, 92-106.

Bruce, E. N. (1988) Correlated and uncorrelated high-frequency oscillations in phrenic and recurrent laryngeal neurograms. Journal of Neurophysiology 59, 1188-1203.

Brunner, M. J., Sussman, M. S., Greene, A. S., Kallman, C. H. and Shoukas, A. A. (1982) Carotid sinus baroreceptor reflex control of respiration. Circulation Research 51, 624-636.

Cerutti, C., Gustin, M. P., Paultre, C. Z., Lo, M., Julien, C., Vincent, M. and Sassard, J. (1991) Autonomic nervous system and cardiovascular variability in rats: a spectral analysis approach. American Journal of Physiology-Heart and Circulatory Physiology 261, H1292-H1299.

Cerutti, C., Lo, M., Julien, C., Paultre, C. Z., Vincent, M. and Sassard, J. (1991) Role of sympathetic nervous system on blood pressure and heart rate variabilities in the rat: spectral analysis. Archives Des Maladies Du Coeur Et des Vaisseaux 84, 1235-1238.

Cerutti, C., Barres, C. and Paultre, C. (1994) Baroreflex modulation of blood pressure and heart rate variabilities in rats: assessment by spectral analysis. American Journal of Physiology-Heart and Circulatory Physiology 266, H1993-H2000.

Chen, Y., Marchenko, V. and Rogers, R. F. (2011) Pulmonary stretch receptor spike time precision increases with lung inflation amplitude and airway smooth muscle tension. Journal of Neurophysiology 105, 2590-2600.

Chen, Y., Marchenko, V. and Rogers, R. F. (2010) Slowly adapting pulmonary stretch receptor spike patterns carry lung distension information. Neuroscience Letters 484, 86-91.

Chen, Y., Marchenko, V. and Rogers, R. F. (2008) Sparse firing frequencybased neuron spike train classification. Neuroscience Letters 439, 47-51.

Chevalier-Cholat, A. M. and Friggi, A. (1977) Carotid sinus, aortic and subclavian baroreceptor activities during cardiopulmonary bypass in rabbits. Journal De Physiologie 72, 971-986.

Chevalier-Cholat, A. M. and Friggi, A. (1976) The reactivity of the sinocarotid, aortic, and subclavian baroreceptors during artificial circulation in the rabbit. Comptes Rendus Hebdomadaires Des Seances De l'Academie Des Sciences. Serie D: Sciences Naturelles 282, 1191-1193. (In French)

Christakos, C. N., Cohen, M. I., See, W. R. and Barnhardt, R. (1988) Fast rhythms in the discharges of medullary inspiratory neurons. Brain Research 463, 362-367.

Christakos, C. N., Cohen, M. I., Barnhardt, R. and Shaw, C. F. (1991) Fast rhythms in phrenic motoneuron and nerve discharges. Journal of Neurophysiology 66, 674-687.

Cohen, M. I., Huang, W. X., Barnhardt, R. and See, W. R. (1993) Timing of 
medullary late-inspiratory neuron discharges: vagal afferent effects indicate possible off-switch function. Journal of Neurophysiology 69, 17841787.

Coote, J. H. (2013) Myths and realities of the cardiac vagus. The Journal of Physiology 591, 4073-4085.

da Silva, E. F., Bassi, M., Menani, J. V., Colombari, D. S. A., Zoccal D. B., Pedrino G. R. and Colombari, E. (2019) Carotid bodies contribute to sympathoexcitation induced by acute salt overload. Experimental Physiology 104, 15-27.

Daly, M. dB. (1986) Interactions between respiration and circulation. In, Cherniack, N. S. and Widdicombe, J. G. (eds.) Handbook of Physiology: The Respiratory System, Volume II: Control of Breathing, Part 2, Volume II (pp. 529-594). Bethesda, MD, USA: American Physiological Society.

Dampney, R. A. (1994) Functional organization of central pathways regulating the cardiovascular system. Physiological Reviews 74, 323-364.

Darnall R. A. and Guyenet P. (1990) Respirophasic modulation of pre- and postganglionic lumbar vasomotor sympathetic neurons in the rat. Neuroscience Letters 119, 148-152.

Dawid Milner, M. S., Lara, J. P., López de Miguel, M. P., López-González, M. V., Spyer, K. M. and González-Barón, S. (2003) A5 region modulation of the cardiorespiratory responses evoked from parabrachial cell bodies in the anaesthetised rat. Brain Research 982, 108-118.

de Abreu, R. M., Porta, A., Rehder-Santos, P., Cairo, B., Donisete da Silva, C., De Favari Signini, Sakaguchi, C. A. and Catai, A. M. (2019) Effects of inspiratory muscle-training intensity on cardiovascular control in amateur cyclists. American Journal of Physiology-Regulatory, Integrative and Comparative Physiology 317, R891-R902.

Dergacheva, O., Griffioen, K. J., Neff, R. A. and Mendelowitz, D. (2010) Respiratory modulation of premotor cardiac vagal neurons in the brainstem. Respiratory Physiology \& Neurobiology 174, 102-110.

D'iachenko, I. E. and Preobrazhenskiı̌, N. N. (1984a) Functional differentiation of afferents of the superior laryngeal nerve in the cat. Neirofiziologiia 16, 777-783. (In Russian)

D'iachenko, I. E. and Preobrazhenskiı̌, N. N. (1984b) Modulation of reflex discharges in the respiratory nerves of the cat upon stimulation of superior laryngeal afferents. Neirofiziologiia 16, 553-557. (In Russian)

D'iachenko, I. E. and Preobrazhenskiĭ, N. N. (1991) Responses of bulbospinal neurons during the expiration reflex in cats. Neirofiziologiia $\mathbf{2 3}$ 88-98. (In Russian)

Dick, T. E., Baekey, D. M., Paton, J. F. R., Lindsey, B. G. and Morris, K. F. (2009) Cardio-respiratory coupling depends on the pons. Respiratory Physiology \& Neurobiology 168, 76-85.

Diedrich, A., Porta, A., Barbic, F., Brychta, R. J., Bonizzi, P., Diedrich, L., Cerutti, S., Robertson, D. and Furlan, R. (2009) Lateralization of expression of neural sympathetic activity to the vessels and effects of carotid baroreceptor stimulation. American Journal of Physiology-Heart and Circulatory Physiology 296, H1758-H1765.

Dittler, R. and Garten, S. (1912) The time course of action current in the phrenic nerve and diaphragm with normal innervation. Zeitschrift für Biologie 58, 420-450. (In German)

Dittmar, C. (1873) Uber die Lage des sogenannten Gefässcentrums in der Medulla oblongata. Berlin Verhage Sächsische Akademie der Wissenschaften zu Leipzig Mathematisch-Physische Klasse 25, 449-469. (In German)

Dove, E. L. and Katona, P. G. (1985) Respiratory effects of brief baroreceptor stimuli in the anesthetized dog. Journal of Applied Physiology $\mathbf{5 9}$ 1258-1265.

Duda, P., Kostik, P. G. and Preobrazhenskiǔ, N. N. (1970) Inhibition of synaptic processes in motor neurons in the presence of rhythmic visceromotor influences. Biulleten' Eksperimental'noi Biologii i Meditsiny 62 3-8.

Duda, P., Kostiuk, P. G. and Preobrazhenski1̌, N. N. (1970) Synaptic processes in motor neurons in the course of viscero-somatic reflexes. Biulleten' Eksperimental'noi Biologii i Meditsiny 61, 3-8. (In Russian)

Dutschmann, M., Bautista, T. G., Mörschel, M. and Dick, T. E. (2014) Learning to breathe: Habituation of Hering-Breuer inflation reflex emerges with postnatal brainstem maturation. Respiratory Physiology \& Neurobiology 195, 44-49.

Dutschmann, M. and Herbert, H. (2006) The Kölliker-Fuse nucleus gates the postinspiratory phase of the respiratory cycle to control inspiratory off-switch and upper airway resistance in rat. European Journal of Neuroscience 24, 1071-1084.

Dyavanapalli, J., Byrne, P. and Mendelowitz, D. (2013) Activation of D2like dopamine receptors inhibits GABA and glycinergic neurotransmission to pre-motor cardiac vagal neurons in the nucleus ambiguus. $\mathrm{Neu}$ roscience 247, 213-226.

Eckberg, D. L. (2003) The human respiratory gate. The Journal of Physiology 548, 339-352.

Eckberg, D. L. (2009) Point: Counterpoint: Respiratory sinus arrhythmia is due to a central mechanism vs. respiratory sinus arrhythmia is due to the baroreflex mechanism. Journal of Applied Physiology 106, 1740-1742.

Eckberg, D. L. and Orshan, C. R. (1977) Respiratory and baroreceptor reflex interactions in man. Journal of Clinical Investigations 59, 780-785.

Ellenberger, H. H. and Feldman, J. L. (1988) Monosynaptic transmission of respiratory drive to phrenic motoneurons from brainstem bulbospinal neurons in rats. The Journal of Comparative Neurology 269, 47-57.

Ellenberger, H. H. (1999) Distribution of bulbospinal gamma-aminobutyric acid-synthesizing neurons of the ventral respiratory group of the rat. The Journal of Comparative Neurology 411, 130-144.

Ezure, K. and Manabe, M. (1988) Decrementing expiratory neurons of the Bötzinger complex. Experimental Brain Research 72, 159-166.

Farmer, D. G. S., Dutschmann, M., Paton, J. F. R., Pickering, A. E. and McAllen, R. M. (2016) Brainstem sources of cardiac vagal tone and respiratory sinus arrhythmia. The Journal of Physiology 594, 7249-7265.

Feldman, J. L. and Del Negro, C. A. (2006) Looking for inspiration: new perspectives on respiratory rhythm. Nature Reviews Neuroscience 7 , 232-241.

Fenik, V. B. (1990) Impulse activity of slow-adapting lung receptors during forced breathing. Neirofiziologiia 21, 629-636. (In Russian)

Fenik, V. B. (1992) Mechanoreceptor system of the respiratory center and its role in the control of respiration. Neirofiziologiia 24, 351-368. (In Russian)

Fortuna, M. G., West, G. H., Stornetta, R. L. and Guyenet, P. G. (2008) Botzinger expiratory-augmenting neurons and the parafacial respiratory group. Journal of Neuroscience 28, 2506-2515.

Frank, J. G., Jameson, H. S., Gorini, C., Mendelowitz, D. (2009) Mapping and identification of GABAergic neurons in transgenic mice projecting to cardiac vagal neurons in the nucleus ambiguus using photo-uncaging. Journal of Neurophysiology 101, 1755-1760.

Fukushi, I., Yokota, S. and Okada, Y. (2019) The role of the hypothalamus in modulation of respiration. Respiratory Physiology \& Neurobiology $\mathbf{2 6 5}$ 172-179.

Fuller, D. D., Johnson, S. M., Olson, E. B. and Mitchell, G. S. (2003) Synaptic pathways to phrenic motoneurons are enhanced by chronic intermittent hypoxia after cervical spinal cord injury. The Journal of Neuroscience 23, 2993-3000.

Futuro-Neto, H.A. and Coote, J.H. (1982) Desynchronized sleep-like pattern of sympathetic activity elicited by electrical stimulation of sites in the brainstem. Brain Research 252, 269-276.

Garcia, A. J., Zanella, S., Dashevskiy, T., Khan, S. A., Khuu, M. A., Prabhakar, N. R. and Ramirez, J. (2016) Chronic intermittent hypoxia alters local respiratory circuit function at the level of the preBötzinger complex. Frontiers in Neuroscience 10, 4.

Gatti, P. J., DaSilva, A. M. T. and Gillis, R. A. (1987) Cardiorespiratory effects produced by injecting drugs that affect GABA receptors into nuclei associated with the ventral surface of the medulla. Neuropharmacology 26, 423-431

Gavrovska, A., Bogdanović, V., Reljin, I. and Reljin, B. (2014) Automatic heart sound detection in pediatric patients without electrocardiogram reference via pseudo-affine Wigner-Ville distribution and Haar wavelet lifting. Computer Methods and Programs in Biomedicine 113, 515-528.

Gebber, G. L. and Barman, S. M. (1988) Studies on the origin and generation of sympathetic nerve activity. Clinical and Experimental Hypertension. Part a: Theory and Practice 10,33-44.

Gebber, G. L., Das, M. and Barman, S. M. (2005) Dynamic changes in baroreceptor-sympathetic coupling during the respiratory cycle. Brain Research 1046, 216-223.

Ghali, M. G. Z. (2020) 'Midbrain control of breathing and blood pressure: The role of periaqueductal gray matter and mesencephalic collicular neuronal microcircuit oscillators'. European Journal of Neuroscience (in press). 
Ghali, M. G. Z. and Marchenko, V. (2013) Fast oscillations during gasping and other non-eupneic respiratory behaviors: Clues to central pattern generation. Respiratory Physiology \& Neurobiology 187, 176-182.

Ghali, M. G. Z. (2015) Vagal modulation of pre-inspiratory activity in hypoglossal discharge in the decerebrate rat. Respiratory Physiology \& Neurobiology 215, 47-50.

Ghali, M. G. Z. and Marchenko, V. (2015) Dynamic changes in phrenic motor output following high cervical hemisection in the decerebrate rat. Experimental Neurology 271, 379-389.

Ghali, M. G. Z. and Marchenko, V. (2016b) Effects of vagotomy on hypoglossal and phrenic responses to hypercapnia in the decerebrate rat. Respiratory Physiology \& Neurobiology 232, 13-21.

Ghali, M. G. Z. and Marchenko, V. (2016a) Patterns of phrenic nerve discharge after complete high cervical spinal cord injury in the decerebrate rat. Journal of Neurotrauma 33, 1115-1127.

Ghali, M. G. Z. (2018) Role of the medullary lateral tegmental field in sympathetic control. Journal of Integrative Neuroscience 16, 189-208.

Ghali, M. G. Z. (2017a) The brainstem network controlling blood pressure. Journal of Hypertension 35, 1938-1947.

Ghali, M. G. Z. (2017b) The bulbospinal network controlling the phrenic motor system: Laterality and course of descending projections. Neuroscience Research 121, 7-17.

Ghali, M. Z. (2017c) The crossed phrenic phenomenon. Neural Regeneration Research 12,845.

Ghali, M. G. Z. (2018) Phrenic motoneurons: output elements of a highly organized intraspinal network. Journal of Neurophysiology 119, 10571070.

Ghali, M. G. Z. (2019a) Dynamic changes in arterial pressure following high cervical transection in the decerebrate rat. The Journal of Spinal Cord Medicine 262, 1-12.

Ghali, M. G. Z. (2019b) Rubral modulation of breathing. Experimental Physiology 104, 1595-1604.

Ghali, M. G. Z. (2019c) Mechanisms contributing to the genesis of hypoglossal preinspiratory discharge. Journal of Integrative Neuroscience 18, 313 325.

Ghali, M. G. Z. and Beshay, S. (2019) Role of fast inhibitory synaptic transmission in neonatal respiratory rhythmogenesis and pattern formation. Molecular and Cellular Neuroscience 100, 103400.

Ghali, M. G. Z. and Ghali, G. Z. (2020) Mechanisms contributing to the generation of mayer waves. Frontiers in Neuroscience 14, 395.

Ghali, M. G. Z. and Marchenko, V. (2016b) Effects of vagotomy on hypoglossal and phrenic responses to hypercapnia in the decerebrate rat. Respiratory Physiology \& Neurobiology 232, 13-21.

Ghali, M. G. Z. (2018) Phrenic motoneurons: output elements of a highly organized intraspinal network. Journal of Neurophysiology 119, 1057 1070.

Gilbey, M. P. (2007) Sympathetic rhythms and nervous integration. Clinical and Experimental Pharmacology and Physiology 34, 356-361.

Gilbey, M. P., Futuro-Neto, H. and Zhou, S. (1995) Respiratory-related discharge patterns of caudal raphe neurones projecting to the upper thoracic spinal cord in the rat. Journal of the Autonomic Nervous System 50, 263 273.

Gilbey, M. P., Jordan, D., Richter, D. W. and Spyer, K. M. (1984) Synaptic mechanisms involved in the inspiratory modulation of vagal cardioinhibitory neurones in the cat. The Journal of Physiology 356, 65-78.

Gilbey, M. P., Numao, Y. and Spyer, K. M. (1986) Discharge patterns of cervical sympathetic preganglionic neurones related to central respiratory drive in the rat. The Journal of Physiology 378, 253-265.

Gillis, R. (1995) Sympathetic nervous system mediated cardiovascular effects of cocaine are primarily due to a peripheral site of action of the drug. Drug and Alcohol Dependence 37, 217-230.

Goodchild, A. K. and Moon, E. A. (2009) Maps of cardiovascular and respiratory regions of rat ventral medulla: Focus on the caudal medulla. Journal of Chemical Neuroanatomy 38, 209-221.

Gordon, F. J. and McCann, L. A. (1988) Pressor responses evoked by microinjections of l-glutamate into the caudal ventrolateral medulla of the rat. Brain Research 457, 251-258.

Gourévitch, B. and Mellen, N. (2014) The preBötzinger complex as a hub for network activity along the ventral respiratory column in the neonate rat. Neuroimage $\mathbf{9 8 , 4 6 0 - 4 7 4 . ~}$
Gray, P. A., Hayes, J. A., Ling, G. Y., Llona, I., Tupal, S., Picardo, M. C. D., Ross, S. E., Hirata, T., Corbin, J. G., Eugenín, J. and Del Negro, C. A. (2010) Developmental origin of preBötzinger complex respiratory neurons. The Journal of Neuroscience 30, 14883-14895.

Grunstein, M. M., Wyszogrodski, I. and Milic-Emili, J (1975) Regulation of frequency and depth of breathing during expiratory threshold loading in cats. Journal of Applied Physiology 38, 869-874.

Guyenet, P. G. and Koshiya, N. (1995) Working model of the sympathetic chemoreflex in rats. Clinical and Experimental Hypertension 17, $167-$ 179.

Guyenet, P. G. (2000) Neural structures that mediate sympathoexcitation during hypoxia. Respiration Physiology 121, 147-162.

Guyenet, P. G., Darnall, R. A. and Riley, T. A. (1990) Rostral ventrolateral medulla and sympathorespiratory integration in rats. American Journal of Physiology-Regulatory, Integrative and Comparative Physiology 259, R1063-R1074.

Guyton, A. C. and Harris, J. W. (1951) Pressoreceptor-autonomic oscillation; a probable cause of vasomotor waves. American Journal of Physiology 165, 158-166.

Guyenet, P. G., Koshiya, N., Huangfu, D., Verberne, A. J. and Riley, T. A. (1993) Central respiratory control of A5 and A6 pontine noradrenergic neurons. American Journal of Physiology-Regulatory, Integrative and Comparative Physiology 264, R1035-R1044.

Guertzenstein, P. G. and Silver, A. (1974) Fall in blood pressure produced from discrete regions of the ventral surface of the medulla by glycine and lesions. The Journal of Physiology 242, 489-503.

Guyenet, P. G., Stornetta, R. L., Holloway, B. B., Souza, G. M. P. R. and Abbott, S. B. G. (2018) Rostral ventrolateral medulla and hypertension. Hypertension 72, 559-566.

Guyenet, P. G., Stornetta, R. L., Souza, G. M. P. R., Abbott, S. B. G., Shi, Y. and Bayliss, D. A. (2019) The retrotrapezoid nucleus: Central chemoreceptor and regulator of breathing automaticity. Trends in Neurosciences 42, 807-824.

Guyenet, P. G. (2006) The sympathetic control of blood pressure. Nature Reviews Neuroscience 7, 335-346.

Guyton, A. C., Batson, H. M., Smith, C. M. and Armstrong, G. G. (1951) Method for studying competence of the body's blood pressure regulatory mechanisms and effect of pressoreceptor denervation. American Journal of Physiology-Legacy Content 164, 360-368.

Guyton, A. C. and Harris, J. W. (2004) Pressoreceptor-autonomic oscillation; a probable cause of vasomotor waves. The American Journal of Physiology 165, 158-166.

Hales, S. (1987) Statical essays: concerning haemastaticks; or, an account of some hydraulick and hydrostatical experiments made on the blood and blood-vessels of animals. London, United Kingdom: Classics of Cardiology Library.

Hales, S. (2003) Foundations of anesthesiology. An account of some hydraulic and hydrostatical experiments made on the blood and bloodvessels of animals. 1710 [classical article]. Journal of Clinical Monitoring and Computing 16, 45-47.

Haselton, J. R. and Guyenet, P. G. (1989) Central respirophasic modulation of medullary sympathoexcitatory neurons in rat. American Journal of Physiology 256, R739-R750.

Hayward, L. F., Castellanos, M. and Davenport, P. W. (2004) Parabrachial neurons mediate dorsal periaqueductal gray evoked respiratory responses in the rat. Journal of Applied Physiology 96, 1146-1154.

Hering, E. (1868). Die Selbststeurung der Athmung durch den Nervus Vagus. Akademie der Wissenschaften Wien (II) 57, 672-677. (In German)

Hering, E. (1869) Uber den Einfluß der Atmung auf den Kreislauf. Akademie der Wissenschaften Wien Mathematisch-Naturwissenschaftliche Klasse 60, 829-856. (In German)

Hernandez, J. P., Xu, F. and Frazier, D. T. (2004) Medial vestibular nucleus mediates the cardiorespiratory responses to fastigial nuclear activation and hypercapnia. Journal of Applied Physiology 97, 835-842.

Hesse, W., Möller, E., Arnold, M. and Schack, B. (2003) The use of timevariant EEG Granger causality for inspecting directed interdependencies of neural assemblies. Journal of Neuroscience Methods 124, 27-44.

Horiuchi, J., McDowall, L. M. and Dampney, R. A. L. (2009) Vasomotor and respiratory responses evoked from the dorsolateral periaqueductal grey are mediated by the dorsomedial hypothalamus. The Journal of Physiology 587, 5149-5162. 
Horn, E. M. and Waldrop, T. G. (1997) Oxygen-sensing neurons in the caudal hypothalamus and their role in cardiorespiratory control. Respiration Physiology 110, 219-228.

Huang, Z., Subramanian, S. H., Balnave, R. J., Turman, A. B. and Moi Chow, C. (2000) Roles of periaqueductal gray and nucleus tractus solitarius in cardiorespiratory function in the rat brainstem. Respiration Physiology 120, 185-195.

Iakunin, V. E., Majskiı̌, V. A., Preobrazhenskiı̆, N. N., Gokin, A. P. and Savos'kina, L. A. (1982) Study of the connections of brain stem respiratory nuclei by the technic of retrograde transport of horseradish peroxidase. Neirofiziologiia 14, 149-157. (In Russian)

Iigaya, K., Horiuchi, J., McDowall, L. M. and Dampney, R. A. L. (2010) Topographical specificity of regulation of respiratory and renal sympathetic activity by the midbrain dorsolateral periaqueductal gray. American Journal of Physiology-Regulatory, Integrative and Comparative Physiology 299, R853-R861.

Iigaya, K., Müller-Ribeiro, F. C. D. F., McDowall, L. M., Nalivaiko, E., Fontes, M. A. P. and Dampney, R. A. L. (2012) Synchronized activation of sympathetic vasomotor, cardiac, and respiratory outputs by neurons in the midbrain colliculi. American Journal of Physiology-Regulatory, Integrative and Comparative Physiology 303, R599-R610.

Janczewski, W. A. and Feldman, J. L. (2006) Distinct rhythm generators for inspiration and expiration in the juvenile rat. The Journal of Physiology 570, 407-420.

Janczewski, W. A., Onimaru, H., Homma, I. and Feldman, J. L. (2002) Opioid-resistant respiratory pathway from the preinspiratory neurones to abdominal muscles:in vivoandin vitrostudy in the newborn rat. The Journal of Physiology 545, 1017-1026.

Janczewski, W. A., Tashima, A., Hsu, P., Cui, Y. and Feldman, J. L. (2013) Role of inhibition in respiratory pattern generation. Journal of Neuroscience 33, 5454-5465.

Jean, A. (1992) The nucleus tractus solitarius: neuroanatomic, neurochemical and functional aspects. Archives Internationales De Physiologie, De Biochimie Et De Biophysique 99, A3-52.

Jiang, C. and Lipski, J. (1990) Extensive monosynaptic inhibition of ventral respiratory group neurons by augmenting neurons in the Bötzinger complex in the cat. Experimental Brain Research 81, 639-648.

Johnson, C. D. and Gilbey, M. P. (1994) Sympathetic activity recorded from the rat caudal ventral artery in vivo. The Journal of Physiology 476, 437442.

Jordan, D., Khalid, M. E. M., Schneiderman, N. and Spyer, K. M. (1982) The location and properties of preganglionic vagal cardiomotor neurones in the rabbit. Pflügers Archiv - European Journal of Physiology 395, 244250.

Julien, C. (2006) The enigma of Mayer waves: Facts and models. Cardiovascular Research 70, 12-21.

Kandel, E. R., Schwartz, J. H. and Jessell T. M (2000) Principles of neural science 4/e. New York: McGraw Hill.

Khan, R. L., Vadigepalli, R., McDonald, M. K., Rogers, R. F., Gao, G. R. and Schwaber, J. S. (2008) Dynamic transcriptomic response to acute hypertension in the nucleus tractus solitarius. American Journal of PhysiologyRegulatory, Integrative and Comparative Physiology 295, R15-R27.

Kidd, C., Malpus, C. M. and Penna, P. E. (1974) Proceedings: An isolated, autoperfused pouch of the subclavian-brachiocephalic baroreceptor area. The Journal of Physiology 238, 19P-20P.

Koba, S., Hanai, E., Kumada, N., Kataoka, N., Nakamura, K., and Watanabe, T. (2018) Sympathoexcitation by hypothalamic paraventricular nucleus neurons projecting to the rostral ventrolateral medulla. The Journal of Physiology 596, 4581-4595.

Koshiya, N. and Guyenet, P. G. (1994) Role of the pons in the carotid sympathetic chemoreflex. American Journal of Physiology-Regulatory, Integrative and Comparative Physiology 267, R508-R518.

Koshiya, N. and Guyenet, P. G. (1995) Sympatholytic effect of clonidine depends on the respiratory phase in rat splanchnic nerve. Journal of the Autonomic Nervous System 53, 82-86.

Koshiya, N. and Guyenet, P. G. (1996) Tonic sympathetic chemoreflex after blockade of respiratory rhythmogenesis in the rat. The Journal of Physiology 491, 859-869.

Kostiuk, P. G. and Preobrazhenskiı̌, N. N. (1966) Differentiation of reciprocal and nonspecific descending synaptic influences during stimulation of the reticular formation of the medulla oblongata. Fiziolohichnyi zhurnal
12, 712-720. (In Ukrainian)

Kostiuk, P. G. and Preobrazhenskii, N. N. (1967) Inhibition of polysynaptic reflex responses during stimulation of the medullary reticular formation. Fiziolohichnyi zhurnal SSSR imeni I. M. Sechenova 53, 1048-1055. (In Russian)

Kostiuk, P. G. and Preobrazhenskiı̌, N. N. (1970) Supraspinal control of synaptic processes during viscero-motor reflexes. Biulleten' Eksperimental'noi Biologii i Meditsiny 62, 3-7.

Kupriyanov, S. V. (2009) Role of baroreceptors in the zone of vertebral arteries in the reflex regulation of venous tone in the splanchnic basin. Bulletin of Experimental Biology and Medicine 148, 9-11.

Lacuey, N., Zonjy, B., Londono, L. and Lhatoo, S. D. (2017) Amygdala and hippocampus are symptomatogenic zones for central apneic seizures. Neurology 88, 701-705.

Lacuey, N., Hampson, J. P., Harper, R. M., Miller, J. P. and Lhatoo, S. (2019) Limbic and paralimbic structures driving ictal central apnea. Neurology 92, e655-e669.

Lahiri, S., Roy, A., Baby, S. M., Hoshi, T., Semenza, G. L. and Prabhakar, N. R. (2006) Oxygen sensing in the body. Progress in Biophysics and Molecular Biology 91, 249-286.

Lansdorp, B., Hofhuizen, C., van Lavieren, M., van Swieten, H., Lemson, J., van Putten, M. J. A. M., van der Hoeven, J. G. and Pickkers, P. (2014) Mechanical ventilation-induced intrathoracic pressure distribution and heart-lung interactions*. Critical Care Medicine 42, 1983-1990.

Lemes, E. V. and Zoccal, D. B. (2014) Vagal afferent control of abdominal expiratory activity in response to hypoxia and hypercapnia in rats. Respiratory Physiology \& Neurobiology 203, 90-97.

Li, Z., Morris, K. F., D. M., Shannon, R. and Lindsey, B. G. (1999b) Multimodal medullary neurons and correlational linkages of the respiratory network. Journal of Neurophysiology 82, 188-201.

Li, Z., Morris, K. F., Baekey, D. M. and Lindsey, B. G. (1999a) Responses of simultaneously recorded respiratory-related medullary neurons to stimulation of multiple sensory modalities. Journal of Neurophysiology $\mathbf{8 2}$, 176-187.

Lindsey, B. G., Arata, A., Morris, K. F., Hernandez, Y. M. and Shannon, R. (1998) Medullary raphe neurones and baroreceptor modulation of the respiratory motor pattern in the cat. The Journal of Physiology 512, 863882.

Lindsey, B. G., Hernandez, Y. M., Morris, K. F., Shannon, R. and Gerstein, G. L. (1992) Dynamic reconfiguration of brain stem neural assemblies: respiratory phase-dependent synchrony versus modulation of firing rates. Journal of Neurophysiology 67, 923-930.

Lipski, J., Waldvogel, H. J., Pilowsky, P. and Jiang, C. (1990) GABAimmunoreactive boutons make synapses with inspiratory neurons of the dorsal respiratory group. Brain Research 529, 309-314.

Lipski, J., Kanjhan, R., Kruszewska, B. and Rong, W. (1996a) Properties of presympathetic neurones in the rostral ventrolateral medulla in the rat: an intracellular study. The Journal of Physiology 490, 729-744.

Lipski, J., Kanjhan, R., Kruszewska, B., Rong, W. F. and Smith, M. (1996b) Pre-sympathetic neurones in the rostral ventrolateral medulla of the rat: electrophysiology, morphology and relationship to adjacent neuronal groups. Acta Neurobiologiae Experimentalis 56, 373-384.

Lois, J. H., Rice, C. D. and Yates, B. J. (2009) Neural circuits controlling diaphragm function in the cat revealed by transneuronal tracing. Journal of Applied Physiology 106, 138-152.

Luck, J. C. (1969) Simultaneous recording of vagal and phrenic activity during stimulation of Hering-Breuer reflexes in the rabbit. The Journal of Physiology 203, 39P-40P.

Machado, B. H. (2001) Neurotransmission of the cardiovascular reflexes in the nucleus tractus solitarii of awake rats. Annals of New York Academy of Sciences 940, 179-196.

Machado, B. H. (2006) Neurotransmission of the cardiovascular reflexes in the nucleus tractus solitarii of awake rats. Annals of the New York Academy of Sciences 940, 179-196.

Malpas, S. (1998) The rhythmicity of sympathetic nerve activity. Progress in Neurobiology 56, 65-96.

Malpas, S. C. (2010) Sympathetic nervous system overactivity and its role in the development of cardiovascular disease. Physiological Reviews 90, 513-557. 
Mandel, D. A. and Schreihofer, A. M. (2006) Central respiratory modulation of barosensitive neurones in rat caudal ventrolateral medulla. The Journal of Physiology 572, 881-896.

Marchenko, V. and Sapru, H. N. (2000) Different patterns of respiratory and cardiovascular responses elicited by chemical stimulation of dorsal medulla in the rat. Brain Research 857, 99-109.

Marchenko, V. and Rogers, R. F. (2006a) Time-frequency coherence analysis of phrenic and hypoglossal activity in the decerebrate rat during eupnea, hyperpnea, and gasping. American Journal of PhysiologyRegulatory, Integrative and Comparative Physiology 291, R1430R1442.

Marchenko, V. and Rogers, R. F. (2006b) Selective loss of high-frequency oscillations in phrenic and hypoglossal activity in the decerebrate rat during gasping. American Journal of Physiology-Regulatory, Integrative and Comparative Physiology 291, R1414-R1429.

Marchenko, V. and Rogers, R. F. (2007) Temperature and state dependence of dynamic phrenic oscillations in the decerebrate juvenile rat. American Journal of Physiology-Regulatory, Integrative and Comparative Physiology 293, R2323-R2335.

Marchenko, V. and Rogers, R. F. (2009) GABAAergic and glycinergic inhibition in the phrenic nucleus organizes and couples fast oscillations in motor output. Journal of Neurophysiology 101, 2134-2145.

Marchenko, V., Ghali, M. G. Z. and Rogers, R. F. (2012) Motoneuron firing patterns underlying fast oscillations in phrenic nerve discharge in the rat. Journal of Neurophysiology 108, 2134-2143.

Marchenko, V., Koizumi, H., Mosher, B., Koshiya, N., Tariq, M. F., Bezdudnaya, T. G., Zhang, R., Molkov, Y. I., Rybak, I. A. and Smith, J. C. (2016) Perturbations of respiratory rhythm and pattern by disrupting synaptic inhibition within pre-bötzinger and bötzinger complexes. Eneuro 3, 16.

Marchenko, V., Ghali, M. G. Z. and Rogers, R. F. (2015) The role of spinal GABAergic circuits in the control of phrenic nerve motor output. American Journal of Physiology-Regulatory, Integrative and Comparative Physiology 308, R916-R926.

Marchenko, V. and Rogers, R. F. (2006a) Selective loss of high-frequency oscillations in phrenic and hypoglossal activity in the decerebrate rat during gasping. American Journal of Physiology-Regulatory, Integrative and Comparative Physiology 291, R1414-R1429.

Marchenko, V. and Rogers, R. F. (2006b) Time-frequency coherence analysis of phrenic and hypoglossal activity in the decerebrate rat during eupnea, hyperpnea, and gasping. American Journal of PhysiologyRegulatory, Integrative and Comparative Physiology 291, R1430R1442.

Marchenko, V. and Rogers, R. F. (2007a) Temperature and state dependence of dynamic phrenic oscillations in the decerebrate juvenile rat. American Journal of Physiology-Regulatory, Integrative and Comparative Physiology 293, R2323-R2335.

Marchenko, V. and Rogers, R. F. (2007b) Retention of lung distension information in pump cell spike trains. American Journal of PhysiologyRegulatory, Integrative and Comparative Physiology 293, R343-R353.

Marchenko, V. and Sapru, H. N. (2003) Cardiovascular responses to chemical stimulation of the lateral tegmental field and adjacent medullary reticular formation in the rat. Brain Research 977, 247-260.

Marina, N., Abdala, A. P., Trapp, S., Li, A., Nattie, E. E., Hewinson, J., Smith, J. C., Paton, J. F. R. and Gourine, A. V. (2010) Essential role of Phox $2 \mathrm{~b}$-expressing ventrolateral brainstem neurons in the chemosensory control of inspiration and expiration. Journal of Neuroscience 30, 1246612473.

Markelov, I. M., Karashurov, E. S. and Mart'ianov, S. G. (1991) Role of intrathoracic pressure in changes in the greater and lesser circulation hemodynamics in patients with bronchial asthma. Sovetskaia Meditsina 11, 10-14.

Marlinskiǔ, V. V., Preobrazhenskiı̌, N. N., Seleznev, V. V. and Shumilina, V. F. (1989) Influence of the efferent vestibular system on the vestibulospinal reflexes in the guinea pig. Neirofiziologiia 21, 78-86. (In Russian)

Martin, H. N. and Booker, W. D. (1878) The influence of stimulation of the midbrain upon the respiratory rhythm of the mammal. The Journal of Physiology 1, 370-426.

Massari, V. J., Dickerson, L. W., Gray, A. L., Lauenstein J. M., Blinder K.
J., Newsome J. T., Rodak D. J., Fleming T. J., Gatti P. J., Gillis R. A. (1998) Neural control of left ventricular contractility in the dog heart: synaptic interactions of negative inotropic vagal preganglionic neurons in the nucleus ambiguus with tyrosine hydroxylase immunoreactive terminals. Brain Research 802, 205-220.

Mayer, S. (1876) Studien zur Physiologie des Herzens und der Blutgefässe. Sitzungsberichte Kaiser Akademie der Wissenschaften 74, 281-307. (In German)

McAllen, R. M. (1987) Central respiratory modulation of subretrofacial bulbospinal neurones in the cat. The Journal of Physiology 388, 533-545.

McAllen, R. M. (1986) Action and specificity of ventral medullary vasopressor neurones in the cat. Neuroscience 18, 51-59.

Mendelowitz, D. (1999) Advances in parasympathetic control of heart rate and cardiac function. Physiology 14, 155-161.

Michelini, L. C. (1994) Vasopressin in the nucleus tractus solitarius: a modulator of baroreceptor reflex control of heart rate. Brazilian Journal of Medical and Biological Research Revista Brasileira De Pesquisas Medicas E Biologicas 27, 1017-1032.

Miyawaki, T., Minson, J., Arnolda, L., Chalmers, J., Llewellyn-Smith, I. and Pilowsky, P. (1996) Role of excitatory amino acid receptors in cardiorespiratory coupling in ventrolateral medulla. American Journal of Physiology-Regulatory, Integrative and Comparative Physiology 271, R1221-R1230.

Miyawaki, T., Pilowsky, P., Sun, Q. J., Minson, J., Suzuki, S., Arnolda, L., Llewellyn-Smith, I. and Chalmers, J. (1995) Central inspiration increases barosensitivity of neurons in rat rostral ventrolateral medulla. American Journal of Physiology-Regulatory, Integrative and Comparative Physiology 268, R909-R918.

Miyazaki, M., Tanaka, I. and Ezure, K. (1999) Excitatory and inhibitory synaptic inputs shape the discharge pattern of pump neurons of the nucleus tractus solitarii in the rat. Experimental Brain Research 129, 191200.

Molkov, Y. I., Abdala, A. P. L., Bacak, B. J., Smith, J. C., Paton, J. F. R. and Rybak, I. A. (2010) Late-expiratory activity: Emergence and interactions with the respiratory CPG. Journal of Neurophysiology 104, 2713-2729.

Molkov, Y. I., Rubin, J. E., Rybak, I. A. and Smith, J. C. (2017) Computational models of the neural control of breathing. Wiley Interdisciplinary Reviews: Systems Biology and Medicine 9, e1371.

Molkov, Y. I., Zoccal, D. B., Baekey, D. M., Abdala, A. P. L., Machado, B. H., Dick, T. E., Paton, J. F. R. and Rybak, I. A. (2014) Physiological and pathophysiological interactions between the respiratory central pattern generator and the sympathetic nervous system. Progress in Brain Research 212, 1-23.

Molkov, Y. I., Zoccal, D. B., Moraes, D. J. A., Paton, J. F. R., Machado, B. H. and Rybak, I. A. (2011) Intermittent hypoxia-induced sensitization of central chemoreceptors contributes to sympathetic nerve activity during late expiration in rats. Journal of Neurophysiology 105, 3080-3091.

Molkov, Y. I., Bacak, B. J., Dick, T. E. and Rybak, I. A. (2013) Control of breathing by interacting pontine and pulmonary feedback loops. Frontiers in Neural Circuits 7, 16.

Montano, N., Barman, S. M., Gnecchi-Ruscone, T., Porta, A., Lombardi, F. and Malliani, A. (1996) Role of low-frequency neuronal activity in the medulla in the regulation of the cardiovascular system. Cardiologia (Rome, Italy) 40, 41-46.

Montano, N., Cogliati, C., da Silva, V. J. D., Gnecchi-Ruscone, T., Massimini, M., Porta, A. and Malliani, A. (2000) Effects of spinal section and of positive-feedback excitatory reflex on sympathetic and heart rate variability. Hypertension 36, 1029-1034.

Montano, N., Gnecchi-Ruscone, T., Porta, A., Lombardi, F., Malliani, A. and Barman, S. M. (1996) Presence of vasomotor and respiratory rhythms in the discharge of single medullary neurons involved in the regulation of cardiovascular system. Journal of the Autonomic Nervous System 57, 116-122.

Montano, N., Lombardi, F., Gnecchi Ruscone, T., Contini, M., Finocchiaro, M. L., Baselli, G., Porta, A., Cerutti, S. and Malliani, A. (1992) Spectral analysis of sympathetic discharge, R-R interval and systolic arterial pressure in decerebrate cats. Journal of the Autonomic Nervous System 40, 21-31.

Moore, R. L. (1927) A study of the Hering-Breuer Reflex. The Journal of Experimental Medicine 46, 819-837.

Moraes, D. J. A., Bonagamba, L. G. H., Costa, K. M., Costa-Silva, J. H., 
Zoccal, D. B. and Machado, B. H. (2014a) Short-term sustained hypoxia induces changes in the coupling of sympathetic and respiratory activities in rats. The Journal of Physiology 592, 2013-2033.

Moraes, D. J. A., da Silva, M. P., Bonagamba, L. G. H., Mecawi, A. S., Zoccal, D. B., Antunes-Rodrigues, J., Varanda, W. A. and Machado, B. H. (2013) Electrophysiological properties of rostral ventrolateral medulla presympathetic neurons modulated by the respiratory network in rats. Journal of Neuroscience 33, 19223-19237.

Moraes, D. J. A., Dias, M. B., Cavalcanti-Kwiatkoski, R., Machado, B. H. and Zoccal, D. B. (2012a) Contribution of the retrotrapezoid nucleus/parafacial respiratory region to the expiratory-sympathetic coupling in response to peripheral chemoreflex in rats. Journal of Neurophysiology 108, 882-890.

Moraes, D. J. A., Machado, B. H. and Zoccal, D. B. (2014b) Coupling of respiratory and sympathetic activities in rats submitted to chronic intermittent hypoxia. Progress in Brain Research 212, 25-38.

Moraes, D. J. A., Zoccal, D. B. and Machado, B. H. (2012b) Medullary respiratory network drives sympathetic overactivity and hypertension in rats submitted to chronic intermittent hypoxia. Hypertension 60, 13741380.

Moraes, D. J. A., Zoccal, D. B. and Machado, B. H. (2012c) Sympathoexcitation during chemoreflex active expiration is mediated by l-glutamate in the RVLMBötzinger complex of rats. Journal of Neurophysiology $\mathbf{1 0 8}$, 610-623.

Moreira, T. S., Takakura, A. C., Colombari, E., West, G. H. and Guyenet, P. G. (2007) Inhibitory input from slowly adapting lung stretch receptors to retrotrapezoid nucleus chemoreceptors. The Journal of Physiology $\mathbf{5 8 0}$ 285-300.

Morgado-Valle, C., Baca, S. M. and Feldman, J. L. (2010) Glycinergic pacemaker neurons in preBötzinger complex of neonatal mouse. The Journal of Neuroscience 30, 3634-3639.

Morgado-Valle, C. and Beltran-Parrazal, L. (2017) Respiratory rhythm generation: The whole is greater than the sum of the parts. Advances in Experimental Medicine and Biology 9, 147-161.

Morrison, S. F. (2016) Central neural control of thermoregulation and brown adipose tissue. Autonomic Neuroscience 196, 14-24.

Morrison, S. F., Barman, S. M. and Gebber, G. L. (1984) Baroreceptor influences on cardiac-related sympathetic nerve activity. Brain Research 301, 175-178.

Morrison, S. F. and Gebber, G. L. (1982) Classification of raphe neurons with cardiac-related activity. American Journal of PhysiologyRegulatory, Integrative and Comparative Physiology 243, R49-R59.

Mörschel, M. and Dutschmann, M. (2009) Pontine respiratory activity involved in inspiratory/expiratory phase transition. Philosophical Transactions of the Royal Society B: Biological Sciences 364, 2517-2526.

Mulkey, D. K., Mistry, A. M., Guyenet, P. G. and Bayliss, D. A. (2006) Purinergic $\mathrm{P} 2$ receptors modulate excitability but do not mediate $\mathrm{pH}$ sensitivity of $\mathrm{rtn}$ respiratory chemoreceptors. Journal of Neuroscience $\mathbf{2 6}$ 7230-7233.

Mulkey, D. K., Rosin, D. L., West, G., Takakura, A. C., Moreira, T. S., Bayliss, D. A. and Guyenet, P. G. (2007) Serotonergic neurons activate chemosensitive retrotrapezoid nucleus neurons by a $\mathrm{pH}$-independent mechanism. Journal of Neuroscience 27, 14128-14138.

Neff, R. A., Wang, J., Baxi, S., Evans, C. and Mendelowitz, D. (2003) Respiratory sinus arrhythmia. Circulation Research 93, 565-572.

Nichols, N. L., Hartzler, L. K., Conrad, S. C., Dean, J. B. and Putnam, R. W. (2008) Intrinsic chemosensitivity of individual nucleus tractus solitarius (NTS) and locus coeruleus (LC) Neurons from neonatal rats. Integration in Respiratory Control 143, 348-352.

Numao, Y., Koshiya, N., Gilbey, M. P. and Spyer, K. M. (1987) Central respiratory drive-related activity in sympathetic nerves of the rat: the regional differences. Neuroscience Letters 81, 279-284.

Ogundele, O. M., Lee, C. C. and Francis, J. (2017) Thalamic dopaminergic neurons projects to the paraventricular nucleus-rostral ventrolateral medulla/C1 neural circuit. The Anatomical Record 300, 1307-1314.

Okazaki, M., Takeda, R., Haji, A. and Yamazaki, H. (2001) Glutamic acid decarboxylase-immunoreactivity of bulbar respiratory neurons identified by intracellular recording and labeling in rats. Brain Research 914, 3447.

Onimaru, H., Kumagawa, Y. and Homma, I. (2006) Respiration-related rhythmic activity in the rostral medulla of newborn rats. Journal of Neurophysiology 96, 55-61.

Onimaru, H., Nakamura, S., Ikeda, K., Kawakami, K. and Inoue, T. (2018) Confocal calcium imaging analysis of respiratory-related burst activity in the parafacial region. Brain Research Bulletin 139, 16-20.

Onimaru, H., Tsuzawa, K., Nakazono, Y. and Janczewski, W. A. (2015) Midline section of the medulla abolishes inspiratory activity and desynchronizes pre-inspiratory neuron rhythm on both sides of the medulla in newborn rats. Journal of Neurophysiology 113, 2871-2878.

Ott, M. M., Nuding, S. C., Segers, L. S., O'Connor, R., Morris, K. F. and Lindsey, B. G. (2012) Central chemoreceptor modulation of breathing via multipath tuning in medullary ventrolateral respiratory column circuits. Journal of Neurophysiology 107, 603-617.

Parati, G., Castiglioni, P., Faini, A., Di Rienzo, M., Mancia, G., Barbieri, R. and Saul, J. P. (2019) Closed-loop cardiovascular interactions and the baroreflex cardiac arm: Modulations over the $24 \mathrm{~h}$ and the effect of hypertension. Frontiers in Physiology 10, 477.

Paxinos, G., Watson, C., Pennisi, M. and Topple, A. (1985) Bregma, lambda and the interaural midpoint in stereotaxic surgery with rats of different sex, strain and weight. Journal of Neuroscience Methods 13, 139-143.

Pereira de Souza Neto, E., Custaud, M. A., Frutoso, J., Somody, L., Gharib, C. and Fortrat, J. O. (2001) Smoothed pseudo Wigner-Ville distribution as an alternative to Fourier transform in rats. Autonomic Neuroscience 87, 258-267.

Phillips, J. K., Goodchild, A. K., Dubey, R., Sesiashvili, E., Takeda, M., Chalmers, J., Pilowsky, P. M. and Lipski, J. (2001) Differential expression of catecholamine biosynthetic enzymes in the rat ventrolateral medulla. The Journal of Comparative Neurology 432, 20-34.

Pijn, J. P., Velis, D. N. and Lopes da Silva, F. H. (1992) Measurement of interhemispheric time differences in generalised spike-and-wave. Electroencephalography and Clinical Neurophysiology 83, 169-171.

Pilowsky, P. (1995) Good vibrations? respiratory rhythms in the central control of blood pressure. Clinical and Experimental Pharmacology and Physiology 22, 594-604.

Porta, A., Bari, V., Maria, B. D., Cairo, B., Vaini, E., Malacarne, M., Pagani, M. and Lucini, D. (2018) Peripheral resistance baroreflex during incremental bicycle ergometer exercise: characterization and correlation with cardiac baroreflex. Frontiers in Physiology 9, 688.

Porta, A., Marchi, A., Bari, V., Heusser, K., Tank, J., Jordan, J., Barbic, F. and Furlan, R. (2015) Conditional symbolic analysis detects nonlinear influences of respiration on cardiovascular control in humans. Philosophical Transactions of the Royal Society a: Mathematical, Physical and Engineering Sciences 373, 20140096.

Preiss, G. and Polosa, C. (1974) Patterns of sympathetic neuron activity associated with Mayer waves. American Journal of Physiology-Legacy Content 226, 724-730.

Preobrazhenskiı̆, N. N. and Fenik, V. B. (1989) Impulse activity of slowly adapting pulmonary receptors during respiration. Neirofiziologiia 20, 518-525. (In Russian)

Preobrazhenskiı̌, N. N., Gokin, A. P. and Zadorozhny̌̆, A. G. (1972) Synaptic processes in thoracic motor neurons evoked by visceral impulsation. Neirofiziologiia 4, 286-295. (In Russian)

Preobrazhenskii, N. N. and Iarovitskii, N. V. (1963) Use of mathematical methods for the determination of the impulse activity of central neurons of the brain. Biofizika 8, 387-393.

Preobrazhenskiı̌, N. N., Shumilina, V. F. and Mă̌skiı̌, V. A. (1989) Brain stem neurons of the guinea pig that form projections into the ampullae of the anterior, lateral and posterior semicircular canals. Neirofiziologiia 20, 526-532. (In Russian)

Preobrazhenskiŭ, N. N. and Tamarova, Z. A. (1970) Intersegmentary routes of the visceralmotor reflexes of the spinal cord. Biulleten' Eksperimental'noi Biologii i Meditsiny 62, 3-8.

Preobrazhenskiı̌, N. N. and Tamarova, Z. A. (1966) Suprasegmentary control of the intraspinal pathways of visceromotor reflexes. Bulletin of Experimental Biology and Medicine 62, 1081-1084.

Preobrazhenskiı̌, N. N. (1966) Microelectrode recording of activity from neurons in vasomotor center. Federation Proceedings. Translation Supplement; Selected Translations from Medical-Related Science 25, 18-22.

Ramirez, J. and Baertsch, N. A. (2018) The dynamic basis of respiratory rhythm generation: One breath at a time. Annual Review of Neuroscience 41, 475-499. 
Richardson, C. A. and Mitchell, R. A. (1982) Power spectral analysis of inspiratory nerve activity in the decerebrate cat. Brain Research 233, 317 336.

Richter, D. W. (1982) Generation and maintenance of the respiratory rhythm. Journal of Experimental Biology 100, 93-107.

Richter, D. W. and Spyer, K. M. (1990) Cardiorespiratory control. In, Loewy, A. D. and Spyer, K. M. (eds.) Central Regulation of Autonomic Functions. New York: Oxford University Press.

Richter, D. W., Jordan, D., Ballantyne, D., Meesmann, M. and Spyer, K. M. (1986) Presynaptic depolarization in myelinated vagal afferent fibres terminating in the nucleus of the tractus solitarius in the cat. Pflügers Archiv - European Journal of Physiology 406, 12-19.

Rogers, R. F., Rybak, I. A. and Schwaber, J. S. (2000) Computational modeling of the baroreflex arc: nucleus tractus solitarius. Brain Research Bulletin 51, 139-150.

Romaniuk, J. R. and Bruce, E. N. (1991) The role of midline ventral medullary structures in generation of respiratory motor high frequency oscillations. Brain Research 565, 148-153.

Rubini, R., Porta, A., Baselli, G., Cerutti, S. and Paro, M. (1993) Power spectrum analysis of cardiovascular variability monitored by telemetry in conscious unrestrained rats. Journal of the Autonomic Nervous System 45, 181-190.

Ruit, K. G. and Neafsey, E. J. (1988) Cardiovascular and respiratory responses to electrical and chemical stimulation of the hippocampus in anesthetized and awake rats. Brain Research 457, 310-321.

Rybak, I. A., Abdala, A. P. L., Markin, S. N., Paton, J. F. R. and Smith, J. C. (2007) Spatial organization and state-dependent mechanisms for respiratory rhythm and pattern generation. Progress in Brain Research 165, 201-220.

Rybak, I. A., Molkov, Y. I., Jasinski, P. E., Shevtsova, N. A. and Smith, J. C. (2014) Rhythmic bursting in the Pre-Bötzinger complex. Progress in Brain Research 209, 1-23.

Schmid, K., Böhmer, G. and Fallert, M. (1988) Influence of rubrospinal tract and the adjacent mesencephalic reticular formation on the activity of medullary respiratory neurons and the phrenic nerve discharge in the rabbit. Pflügers Archiv - European Journal of Physiology 413, 23-31.

Schroeder, W. and Brehm, H. (1952) Untersuchungen über den adäquaten Reiz des Bainbridge-Reflexes. Pflügers Archiv Gesamte Physiology Menschen Tiere 255, 114-129. (In German)

Schroeder, W. and Brehm, H. (2004) Adequate stimulation for the Bainbridge reflex. Pflugers Archiv Fur Die Gesamte Physiologie des Menschen Und der Tiere 255, 114-129.

Schweitzer, A. (1945) Rhythmical fluctuations of the arterial blood pressure. Journal of Physiology 104, 25P.

Schreiber, T. (2000) Measuring Information Transfer. Physical Review Letters $\mathbf{8 5}, 461-464$

Schwaber, J. S., Graves, E. B. and Paton, J. F. R. (1993) Computational modeling of neuronal dynamics for systems analysis: application to neurons of the cardiorespiratory NTS in the rat. Brain Research 604, 126-141.

Seals, D. R., Suwarno, N. O., Joyner, M. J., Iber, C., Copeland, J. G. and Dempsey, J. A. (1993) Respiratory modulation of muscle sympathetic nerve activity in intact and lung denervated humans. Circulation Research 72, 440-454.

Seyedabadi, M. (2006) A novel pressor area at the medullo-cervical junction that is not dependent on the RVLM: Efferent pathways and chemical mediators. Journal of Neuroscience 26, 5420-5427.

Seydnejad, S. R. and Kitney, R. I. (2001) Modeling of Mayer waves generation mechanisms. IEEE Engineering in Medicine and Biology Magazine 20, 92-100.

Shumilina, V. F., Preobrazhenskiı̌, N. N. and Maĭskǐ̌, V. A. (1987) Study of vestibular efferent neurons of the guinea pig by the technic of retrograde axonal transport of horseradish peroxidase and with fluorochromes. Neirofiziologiia 18, 738-747. (In Russian)

Shumilina, V. F. and Preobrazhenskiı̆, N. N. (1991) Vestibular efferent neurons of the guinea pig forming projections into the saccule. Neirofiziologiia 22, 657-665. (In Russian)

Smirnov, D. A., Barnikol, U. B., Barnikol, T. T., Bezruchko, B. P., Hauptmann, C., Bührle, C., Maarouf, M., Sturm, V., Freund, H. and Tass, P. A. (2008) The generation of Parkinsonian tremor as revealed by directional coupling analysis. Epl (Europhysics Letters) 83, 20003

Smith, J. C., Ellenberger, H. H., Ballanyi, K., Richter, D. W. and Feldman, J.
L. (1991) Pre-Bötzinger complex: a brainstem region that may generate respiratory rhythm in mammals. Science 254, 726-729.

Smith, J. C., Abdala, A. P. L., Koizumi, H., Rybak, I. A. and Paton, J. F. R. (2007) Spatial and functional architecture of the mammalian brain stem respiratory network: A hierarchy of three oscillatory mechanisms. Journal of Neurophysiology 98, 3370-3387.

Smith, J. C., Abdala, A. P. L., Rybak, I. A. and Paton, J. F. R. (2009) Structural and functional architecture of respiratory networks in the mammalian brainstem. Philosophical Transactions of the Royal Society B: Biological Sciences 364, 2577-2587.

Sobrinho, C. R., Wenker, I. C., Poss, E. M., Takakura, A. C., Moreira, T. S. and Mulkey, D. K. (2014) Purinergic signalling contributes to chemoreception in the retrotrapezoid nucleus but not the nucleus of the solitary tract or medullary raphe. The Journal of Physiology 592, 1309-1323.

St. Croix, C. M., Satoh, M., Morgan, B. J., Skatrud, J. B. and Dempsey, J. A. (1999) Role of respiratory motor output in within-breath modulation of muscle sympathetic nerve activity in humans. Circulation Research $\mathbf{8 5}$, 457-469.

Subramanian, H. H., Balnave, R. J. and Holstege, G. (2008a) The midbrain periaqueductal gray control of respiration. Journal of Neuroscience $\mathbf{2 8}$ 12274-12283.

Subramanian, H., Huang, Z. and Balnave, R. (2008b) Responses of brainstem respiratory neurons to activation of midbrain periaqueductal gray in the rat. Integration in Respiratory Control 94, 377-381.

Subramanian, H. H. and Holstege, G. (2014) The midbrain periaqueductal gray changes the eupneic respiratory rhythm into a breathing pattern necessary for survival of the individual and of the species. Progress in Brain Research 212, 351-384.

Sun, Q., Minson, J., Llewellyn-Smith, I. J., Arnolda, L., Chalmers, J. and Pilowsky, P. (1997) Bötzinger neurons project towards bulbospinal neurons in the rostral ventrolateral medulla of the rat. The Journal of Comparative Neurology 388, 23-31.

Sun, M. K. and Guyenet, P. G. (1986) Medullospinal sympathoexcitatory neurons in normotensive and spontaneously hypertensive rats. American Journal of Physiology-Regulatory, Integrative and Comparative Physiology 250, R910-R917.

Sun, M., Young, B. S., Hackett, J. T. and Guyenet, P. G. (1988) Reticulospinal pacemaker neurons of the rat rostral ventrolateral medulla with putative sympathoexcitatory function: an intracellular study in vitro. Brain Research 442, 229-239.

Sysoev, I. V., Ponomarenko, V. I., Kulminskiy, D. D. and Prokhorov, M. D. (2016) Recovery of couplings and parameters of elements in networks of time-delay systems from time series. Physical Review E 94, 052207.

Sysoeva, M. V., Sitnikova, E., Sysoev, I. V., Bezruchko, B. P. and van Luijtelaar, G. (2014) Application of adaptive nonlinear Granger causality: disclosing network changes before and after absence seizure onset in a genetic rat model. Journal of Neuroscience Methods 226, 33-41.

Takakura, A. C., Moreira, T. S., West, G. H., Gwilt, J. M., Colombari, E., Stornetta, R. L. and Guyenet, P. G. (2007) GABAergic pump cells of solitary tract nucleus innervate retrotrapezoid nucleus chemoreceptors. Journal of Neurophysiology 98, 374-381.

Tağluk, M. E., Cakmak, E. D. and Karakas, S. (2005) Analysis of the timevarying energy of brain responses to an oddball paradigm using shortterm smoothed Wigner-Ville distribution. Journal of Neuroscience Methods 143, 197-208.

Taylor, E. W., Leite, C. A. C. and Levings, J. J. (2009) Central control of cardiorespiratory interactions in fish. Acta Histochemica 111, 257-267.

Terui, N., Saeki, Y. and Kumada, M. (1986) Barosensory neurons in the ventrolateral medulla in rabbits and their responses to various afferent inputs from peripheral and central sources. The Japanese Journal of Physiology 36, 1141-1164.

Tian, G. F. and Duffin, J. (1996a) Connections from upper cervical inspiratory neurons to phrenic and intercostal motoneurons studied with crosscorrelation in the decerebrate rat. Experimental Brain Research 110, 196204.

Tian, G. F. and Duffin, J. (1996b) Spinal connections of ventral-group bulbospinal inspiratory neurons studied with cross-correlation in the decerebrate rat. Experimental Brain Research 111, 178-186.

Toorop, R. J., Ousrout, R., Scheltinga, M. R. M., Moll, F. L. and Bleys, R. L. A. W. (2013) Carotid baroreceptors are mainly localized in the me- 
dial portions of the proximal internal carotid artery. Annals of Anatomy - Anatomischer Anzeiger 195, 248-252.

Traube, L. (1865) Uber periodische thatigkeits-aeusserungen des vasomotorishen un hemmungs-nervenzentrums. Centralblatt fur die medicinischen Wissenschaften 56, 881-885. (In German)

Ursino, M., Fabbri, G. and Belardinelli, E. (1992) A mathematical analysis of vasomotion in the peripheral vascular bed. Cardioscience 3, 13-25.

van Brederode, J. F. and Berger, A. J. (2011) GAD67-GFP ${ }^{+}$neurons in the Nucleus of Roller. II. Subthreshold and firing resonance properties. Journal of Neurophysiology 105, 249-278.

van de Borne, P., Rahnama, M., Mezzetti, S., Montano, N., Porta, A., Degaute, J. P. and Somers, V. K. (2001) Contrasting effects of phentolamine and nitroprusside on neural and cardiovascular variability. American Journal of Physiology-Heart and Circulatory Physiology 281, H559H565.

Vatner, S. F. and Zimpfer, M. (1981) Bainbridge reflex in conscious, unrestrained, and tranquilized baboons. American Journal of PhysiologyHeart and Circulatory Physiology 240, H164-H167.

Von Euler, C., Hayward, J. N., Marttila, I. and Wyman, R. J. (1973) Respiratory neurones of the ventrolateral nucleus of the solitary tract of cat: Vagal input, spinal connections and morphological identification. Brain Research 61, 1-22.

Wallin, B. G. and Eckberg, D. L. (1982) Sympathetic transients caused by abrupt alterations of carotid baroreceptor activity in humans. American Journal of Physiology-Heart and Circulatory Physiology 242, H185H190.

Wasserman, A. M., Ferreira, M., Sahibzada, N., Hernandez, Y. M. and Gillis, R. A. (2002) GABA-mediated neurotransmission in the ventrolateral NTS plays a role in respiratory regulation in the rat. American Journal of Physiology-Regulatory, Integrative and Comparative Physiology 283, R1423-R1441.

Watanabe, K., Ooishi, Y. and Kashino, M. (2015) Sympathetic tone induced by high acoustic tempo requires fast respiration. PLoS one 10, e0135589.

Wyss, O. A. (2003) Synchronization of inspiratory motor activity as compared between phrenic and vagus nerve. The Yale Journal of Biology and Medicine 28, 471-480.

Zaki Ghali, M. G., Britz, G. and Lee, K. (2019) Pre-phrenic interneurons: Characterization and role in phrenic pattern formation and respiratory recovery following spinal cord injury. Respiratory Physiology \& Neurobiology 265, 24-31.

Zhang, Z., Zhuang, J., Zhang, C. and Xu, F. (2011) Activation of opioid $\mu$-receptors in the commissural subdivision of the nucleus tractus solitarius abolishes the ventilatory response to hypoxia in anesthetized rats. Anesthesiology 115, 353-363.

Zhao, H., Wang, L., Hua, S. and Wang, S. (1997) Effects of NPY microionophoresis on the units in nucleus tractus solitarius (NTS) responsive to baro-and chemoreceptor. Chinese Journal of Applied Physiology 13, 333-336. (In Chinese)

Zhdanov, V. A. (1984) Significance of the lung mechanoreceptors in short- ening expiration during lung collapse. Fiziologicheskii Zhurnal SSSR Imeni i. M. Sechenova 70, 186-194. (In Russian)

Zhdanov, V. A., Obukhova, E. A., Efremova, E. V. and Zarnadze, N. R. (1989) The relative amount of slow-adapting stretch receptors in the trachea, extrapulmonary bronchi and lungs of the cat. Fiziologicheskii Zhurnal SSSR Imeni i. M. Sechenova 75, 955-962. (In Russian)

Zhigă̌lo, T. L., Nuridzhanova, A. A. and Preobrazhenskiı̌, N. N. (1985) Electrophysiological characteristics of the bulbar respiratory neurons. Fiziologicheskii Zhurnal 30, 660-667. (In Russian)

Zhong, S., Gebber, G. L., Zhou, S. and Barman, S. M. (1998) Nonlinear interactions of slow and rapid rhythms in sympathetic nerve discharge. American Journal of Physiology-Heart and Circulatory Physiology 275 , H331-H340.

Zhou, S. Y. and Gilbey, M. P. (1992) Respiratory-related activity of lower thoracic and upper lumbar sympathetic preganglionic neurones in the rat. The Journal of Physiology 451, 631-642.

Zhou, D., Huang, Q., Fung, M.L., Li, A., Darnall, R. A., Nattie, E. E. and St. John, W. M. (1996) Phrenic response to hypercapnia in the unanesthetized, decerbrate, newborn rat. Respiratory Physiology 104, 11-22.

Zoccal, D. B. (2015) Peripheral chemoreceptors and cardiorespiratory coupling: a link to sympatho-excitation. Experimental Physiology 100, 143148 .

Zoccal, D. B., Bonagamba, L. G. H., Oliveira, F. R. T., Antunes-Rodrigues, J. and Machado, B. H. (2007) Increased sympathetic activity in rats submitted to chronic intermittent hypoxia. Experimental Physiology 92, 7985.

Zoccal, D. B., Bonagamba, L. G. H., Paton, J. F. R. and Machado, B. H. (2009a) Sympathetic-mediated hypertension of awake juvenile rats submitted to chronic intermittent hypoxia is not linked to baroreflex dysfunction. Experimental Physiology 94, 972-983.

Zoccal, D. B. and Machado, B. H. (2011) Coupling between respiratory and sympathetic activities as a novel mechanism underpinning neurogenic hypertension. Current Hypertension Reports 13, 229-236.

Zoccal, D. B., Paton, J. F. and Machado, B. H. (2009b) Do changes in the coupling between respiratory and sympathetic activities contribute to neurogenic hypertension? Clinical and Experimental Pharmacology and Physiology 36, 1188-1196.

Zoccal, D. B., Simms, A. E., Bonagamba, L. G. H., Braga, V. A., Pickering, A. E., Paton, J. F. R. and Machado, B. H. (2008) Increased sympathetic outflow in juvenile rats submitted to chronic intermittent hypoxia correlates with enhanced expiratory activity. The Journal of Physiology 586, 3253-3265.

Zoccal, D. B., Silva, J. N., Barnett, W. H., Lemes, E. V., Falquetto, B., Colombari, E., Molkov, Y. I., Neuroscience Institute, Georgia State University, Atlanta, Georgia, Moreira, T. S. and Takakura, A. C. (2018) Interaction between the retrotrapezoid nucleus and the parafacial respiratory group to regulate active expiration and sympathetic activity in rats. American Journal of Physiology-Lung Cellular and Molecular Physiology 315, L891-L909. 